\title{
Hyperspherical harmonics for tetraatomic systems
}

\author{
Desheng Wang and Aron Kuppermann ${ }^{\text {a) }}$ \\ Arthur Amos Noyes Laboratory of Chemical Physics, Division of Chemistry and Chemical Engineering, \\ California Institute of Technology, Pasadena, California 91125
}

(Received 26 July 2001; accepted 29 August 2001)

\begin{abstract}
A recursion procedure for the analytical generation of hyperspherical harmonics for tetraatomic systems, in terms of row-orthonormal hyperspherical coordinates, is presented. Using this approach and an algebraic Mathematica program, these harmonics were obtained for values of the hyperangular momentum quantum number up to 30 (about 43.8 million of them). Their properties are presented and discussed. Since they are regular at the poles of the tetraatomic kinetic energy operator, are complete, and are not highly oscillatory, they constitute an excellent basis set for performing a partial wave expansion of the wave function of the corresponding Schrödinger equation in the strong interaction region of nuclear configuration space. This basis set is, in addition, numerically very efficient and should permit benchmark-quality calculations of state-to-state differential and integral cross sections for those systems. (0) 2001 American Institute of Physics.

[DOI: $10.1063 / 1.1412603$ ]
\end{abstract}

\section{INTRODUCTION}

Substantial progress has been made recently in applying quantum reactive scattering theory to atom-diatom reactions. Several totally ab initio quantum-dynamical calculations of converged state-to-state integral and differential cross sections have been performed using a propagation approach to solve the time-independent Schrödinger equation. Most of these calculations have been done with some form of symmetrized body-fixed hyperspherical coordinates ${ }^{1-6}$ which have the desirable property that, as opposed to Jacobi coordinates, a single set of such coordinates span all arrangement channels "democratically" (i.e., equivalently). This property has been instrumental in the success of such an approach.

Extension of these kinds of calculations to tetraatomic systems has been difficult. Time-dependent and timeindependent wave-packet methods have been used for the total angular momentum $J=0$ partial wave of such reactions, ${ }^{7-15}$ but for $J>0$ only approximate techniques have been used. No completely converged state-to-state differential cross sections have yet been obtained by these methods. Given that they include, in a single calculation, a broad range of energies, they are not particularly well suited for such calculations because of the very large number of energies and states involved.

An alternative means for performing such $a b$ initio calculations is to use non-wave-packet (i.e., single energy) time-independent methods. One approach of this kind is to employ different sets of hyperspherical coordinates in different regions of configuration space. Such a strategy has recently been successfully applied to the $J=0$ partial wave of the $\mathrm{OH}+\mathrm{H}_{2} \rightarrow \mathrm{H}_{2} \mathrm{O}+\mathrm{H}$ reaction. ${ }^{16}$ It has required overcoming linear dependence problems associated with the super-

\footnotetext{
a) Author to whom correspondence should be addressed. Electronic mail: aron@caltech.edu
}

completeness, in the overlapping regions of configuration space, of the basis set used. This method has constituted a major step forward, but it is not clear yet how practical it will be to extend it to all partial waves needed to generate stateto-state differential cross sections. Recently, a set of bodyfixed row-orthonormal hyperspherical coordinates (ROHC) has been proposed. ${ }^{17-19}$ They are related to the ones used previously by Zickendraht. ${ }^{20}$ The Hamilitonian in these coordinates is quite simple, and each of its terms displays useful invariance properties under kinematic rotations and symmetry operations. ${ }^{17}$ However, as for angular coordinates in general, the corresponding kinetic energy operator has poles for special configurations of the system. For collinearlydominated triatomic reactions there are two such poles; one for noncollinear geometries in regions of configuration space that are classically forbidden at the energies of interest, and the other for collinear geometries, that can be dealt with analytically using a simple set of basis functions which behave properly at that pole. ${ }^{1}$ For noncollinearly-dominated triatomic reactions, the noncollinear pole still presents a problem. For tetraatomic systems this problem is more pronounced. The kinetic energy operator, in the principalaxes-of-inertia body-fixed frame used in association with the $\mathrm{ROHC}$, has a pole for any configuration for which two of the system's three principal moments of inertia are equal. The system's potential energy surface on which the reaction is assumed to occur cannot be expected, in general, to make such configurations fall in classically forbidden regions of configuration space even at low energies. Furthermore, the analytical determination of basis functions that behave properly at these poles has not been possible until now, and their search by numerical approaches has also been unsuccessful. These basis functions can be chosen to be eigenfunctions of the system's grand-canonical angular momentum operator (also called the hyperangular momentum operator), and are called hyperspherical harmonics. The problem is, however, that principal-axes-of-inertia body-fixed hyperspherical har- 
monics are not explicitly known for the wide range of the corresponding quantum number $n$ and of the usual total angular momentum quantum number $J$ needed to perform converged reactive scattering calculations.

Space-fixed (as opposed to body-fixed) hyperspherical harmonics have been extensively used to calculate the energy levels of nuclei ${ }^{21,22}$ and atoms. ${ }^{23}$ An extensive review of their properties has been given by Avery. ${ }^{24}$ The space-fixed frame is however not well suited for reactive scattering, because different coordinates are needed to properly describe the system in different regions of nuclear configuration space, and this leads to overcompleteness problems of the type mentioned above.

In this paper we describe an efficient recursive method to generate, for tetraatomic systems, analytical hyperspherical harmonics in the principal-axes-of-inertia frame which are simultaneous eigenfunctions of the angular momentum operators $\hat{\wedge}^{2}, \hat{J}^{2}, \hat{J}_{z}^{\mathrm{sf}}, \hat{L}^{2}$, and $\hat{L}_{\lambda_{3}}^{\text {bf }} \cdot \hat{\wedge}^{2}$ is the hyperangular momentum operator, $\hat{J}^{2}$ the square of the total angular momentum operator, $\hat{J}_{z}^{\text {sf }}$ its space-fixed $\mathrm{z}$ component, $\hat{L}^{2}$ the square of an internal hyperangular momentum operator associated with arrangement channel coordinates $\lambda$ and $\hat{L}_{\lambda_{3}}^{\text {bf }}$ one of the latter's component in an internal mathematical frame. ${ }^{17}$ In Sec. II we describe these operators in greater detail and summarize the ROHC used and the corresponding Hamiltonian, ${ }^{17}$ and in Sec. III we define the associated hyperspherical harmonics. In Sec. IV we derive the recursion relations used to generate them and in Sec. V their degeneracies are analyzed. Some representative results are presented in Sec. VI and discussed in Sec. VII. Finally, a summary and conclusions are given in Sec. VIII.

\section{COORDINATES AND KINETIC ENERGY OPERATOR}

The ROHC used in this paper, as well as their properties, have been described previously ${ }^{17}$ and we only summarize them below. We consider a system of four bodies in a spacefixed frame $O x_{1}^{\mathrm{sf}} x_{2}^{\mathrm{sf}} x_{3}^{\mathrm{sf}}$ whose mass-scaled $\lambda$-arrangement channel Jacobi vectors are $\mathbf{r}_{\lambda}^{(i)}, i=1,2,3$. The Jacobi matrix can be written as

$$
\boldsymbol{\rho}_{\lambda}^{\mathrm{sf}}=\left(\begin{array}{ccc}
x_{\lambda_{1}}^{(1)} & x_{\lambda_{1}}^{(2)} & x_{\lambda_{1}}^{(3)} \\
x_{\lambda_{2}}^{(1)} & x_{\lambda_{2}}^{(2)} & x_{\lambda_{2}}^{(3)} \\
x_{\lambda_{3}}^{(1)} & x_{\lambda_{3}}^{(2)} & x_{\lambda_{3}}^{(3)}
\end{array}\right) .
$$

The $10 \mathrm{ROHC}$,

$$
\begin{aligned}
& \gamma_{\lambda} \equiv\left(\chi, \rho, \boldsymbol{\Theta}_{\lambda}\right), \\
& \boldsymbol{\Theta}_{\lambda} \equiv\left(\mathbf{a}_{\lambda}, \theta, \phi, \boldsymbol{\delta}_{\lambda}\right)
\end{aligned}
$$

are defined by the relation

$$
\boldsymbol{\rho}_{\lambda}^{\mathrm{sf}}=(-1)^{\chi} \widetilde{\mathbf{R}}\left(\mathbf{a}_{\lambda}\right) \rho \mathbf{N}(\theta, \phi) \widetilde{\mathbf{R}}\left(\boldsymbol{\delta}_{\lambda}\right) .
$$

In this expression, $\mathbf{a}_{\lambda} \equiv\left(a_{\lambda}, b_{\lambda}, c_{\lambda}\right)$ are the Euler angles that rotate the space-fixed frame into the principal-axes-of-inertia frame $O x_{1}^{I \lambda} x_{2}^{I \lambda} x_{3}^{I \lambda}$ and $\chi$ is the chirality variable that can assume the values 0 or 1 and is defined by

$$
(-1)^{\chi}=\operatorname{sign}\left(\operatorname{det} \boldsymbol{\rho}_{\lambda}^{\mathrm{sf}}\right) \text {. }
$$

The quantity $\rho$ is the usual hyperradius and together with $\chi$ and the five internal hyperangles $\theta, \phi, \boldsymbol{\delta}_{\boldsymbol{\lambda}}$ determines the internal configuration of the system. The two $\mathbf{R}$ in Eq. (2.4) are proper rotation matrices and $\mathbf{N}(\theta, \phi)$ is a diagonal matrix whose diagonal elements are

$$
N_{11}=\sin \theta \cos \phi, \quad N_{22}=\sin \theta \sin \phi, \quad N_{33}=\cos \theta .
$$

These Euler angles $\mathbf{a}_{\lambda}$ have the usual ranges of definition,

$$
0 \leqslant a_{\lambda}, c_{\lambda}<2 \pi \quad 0 \leqslant b_{\lambda} \leqslant \pi
$$

In order to get a one-to-one correspondence between $\boldsymbol{\rho}_{\lambda}^{\text {sf }}$ and the $10 \mathrm{ROHC}$ (except for some special geometries), we limit the ranges of the $\delta_{\lambda}^{(i)}(i=1,2,3)$ to

$$
0 \leqslant \delta_{\lambda}^{(1)}, \delta_{\lambda}^{(3)}<\pi \quad 0 \leqslant \delta_{\lambda}^{(2)} \leqslant \pi
$$

and of $\phi$ and $\theta$ to

$$
\begin{aligned}
0 & \leqslant \phi \leqslant \pi / 4 \quad 0 \leqslant \theta \leqslant \arcsin \left[1 /\left(1+\cos ^{2} \phi\right)^{1 / 2}\right. \\
& \left.\leqslant \arcsin (2 / 3)^{1 / 2} \simeq 54.7^{\circ}\right] .
\end{aligned}
$$

The latter results in

$$
0 \leqslant N_{22} \leqslant N_{11} \leqslant N_{33} \text {. }
$$

The kinematic-rotation-invariant hyperangles $\theta$ and $\phi$ are related to system's principal moments of inertia by

$$
\begin{aligned}
& I_{1}=\mu \rho^{2}\left(N_{22}^{2}+N_{33}^{2}\right), \\
& I_{2}=\mu \rho^{2}\left(N_{11}^{2}+N_{33}^{2}\right), \\
& I_{3}=\mu \rho^{2}\left(N_{11}^{2}+N_{22}^{2}\right),
\end{aligned}
$$

and, as a result of Eq. (2.10), are ordered according to

$$
I_{2} \geqslant I_{1} \geqslant I_{3} \geqslant 0 \text {. }
$$

In terms of these $\mathrm{ROHC}$, the kinetic energy operator is given by

$$
\hat{T}=-\frac{\hbar^{2}}{2 \mu} \nabla^{2}=\hat{T}_{\rho}(\rho)+\frac{\hat{\wedge}^{2}}{2 \mu \rho^{2}},
$$

where $\nabla^{2}$ is the nine-dimensional Laplacian, $\hat{\wedge}^{2}$ is the hyperangular momentum operator,

$$
\hat{\wedge}^{2}=\hat{K}^{2}(\theta, \phi)+\hat{B}(\theta, \phi)+\hat{C}^{2}\left(\boldsymbol{\Theta}_{\lambda}\right),
$$

and $\hat{T}_{\rho}(\rho)$ is the hyper-radial kinetic energy operator,

$$
\hat{T}_{\rho}(\rho)=-\frac{\hbar^{2}}{2 \mu} \frac{1}{\rho^{8}} \frac{\partial}{\partial \rho} \rho^{8} \frac{\partial}{\partial \rho} .
$$

The terms in Eq. (2.16) are defined by

$$
\begin{aligned}
& \hat{K}^{2}(\theta, \phi)=-\hbar^{2}\left(\frac{1}{\sin \theta} \frac{\partial}{\partial \theta} \sin \theta \frac{\partial}{\partial \theta}+\frac{1}{\sin ^{2} \theta} \frac{\partial^{2}}{\partial \phi^{2}}\right), \\
& \hat{B}(\theta, \phi)=-2 \hbar^{2}\left[b_{\theta}(\theta, \phi) \frac{\partial}{\partial \theta}+\frac{1}{\sin \theta} b_{\phi}(\theta, \phi) \frac{\partial}{\partial \phi}\right],
\end{aligned}
$$




$$
\begin{gathered}
b_{\theta}(\theta, \phi)=\frac{N_{\theta_{11}}^{\prime} N_{11}-N_{\theta_{22}}^{\prime} N_{22}}{N_{11}^{2}-N_{22}^{2}}+\frac{N_{\theta_{11}}^{\prime} N_{11}-N_{\theta_{33}}^{\prime} N_{33}}{N_{11}^{2}-N_{33}^{2}} \\
+\frac{N_{\theta_{22}}^{\prime} N_{22}-N_{\theta_{33}}^{\prime} N_{33}}{N_{22}^{2}-N_{33}^{2}}, \\
b_{\phi}(\theta, \phi)=\frac{M_{\phi_{11}} N_{11}-M_{\phi_{22}} N_{22}}{N_{11}^{2}-N_{22}^{2}}+\frac{M_{\phi_{11}} N_{11}}{N_{11}^{2}-N_{33}^{2}} \\
+\frac{M_{\phi_{22}} N_{22}}{N_{22}^{2}-N_{33}^{2}},
\end{gathered}
$$

and

$$
\begin{aligned}
\hat{C}^{2}\left(\boldsymbol{\Theta}_{\lambda}\right)= & \frac{\left(N_{22} \hat{J}_{1}^{I_{\lambda}}-N_{33} \hat{L}_{\lambda_{1}}\right)^{2}+\left(N_{33} \hat{J}_{1}^{I_{\lambda}}-N_{22} \hat{L}_{\lambda_{1}}\right)^{2}}{\left(N_{22}^{2}-N_{33}^{2}\right)^{2}} \\
& +\frac{\left(N_{33} \hat{J}_{2}^{I_{\lambda}}-N_{11} \hat{L}_{\lambda_{2}}\right)^{2}+\left(N_{11} \hat{J}_{2}^{I_{\lambda}}-N_{33} \hat{L}_{\lambda_{2}}\right)^{2}}{\left(N_{11}^{2}-N_{33}^{2}\right)^{2}} \\
& +\frac{\left(N_{11} \hat{J}_{3}^{I_{\lambda}}-N_{22} \hat{L}_{\lambda_{3}}\right)^{2}+\left(N_{22} \hat{J}_{3}^{I_{\lambda}}-N_{11} \hat{L}_{\lambda_{3}}\right)^{2}}{\left(N_{11}^{2}-N_{22}^{2}\right)^{2}},
\end{aligned}
$$

where

$$
N_{\theta_{11}}^{\prime}=\cos \theta \cos \phi, \quad N_{\theta_{22}}^{\prime}=\cos \theta \sin \phi, \quad N_{\theta_{33}}^{\prime}=-\sin \theta,
$$

$$
M_{\phi_{11}}=-\sin \phi \quad M_{\phi_{22}}=\cos \phi .
$$

The $\hat{J}_{1}^{I_{\lambda}}, \hat{J}_{2}^{I_{\lambda}}$, and $\hat{J}_{3}^{I_{\lambda}}$ operators in Eq. (2.22) are the components of the nuclear motion angular momentum operator $\hat{\mathbf{J}}$ in the body-fixed frame $O x_{1}^{I_{\lambda}} x_{2}^{I_{\lambda}} x_{3}^{I_{\lambda}}$ and are given explicitly by

$$
\begin{aligned}
\left(\begin{array}{c}
\hat{J}_{1}^{I_{\lambda}} \\
\hat{J}_{2}^{I_{\lambda}} \\
\hat{J}_{3}^{I_{\lambda}}
\end{array}\right)= & \frac{\hbar}{i}\left(\begin{array}{ccc}
-\csc b_{\lambda} \cos c_{\lambda} & \sin c_{\lambda} & \cot b_{\lambda} \cos c_{\lambda} \\
\csc b_{\lambda} \sin c_{\lambda} & \cos c_{\lambda} & -\cot b_{\lambda} \sin c_{\lambda} \\
0 & 0 & 1
\end{array}\right) \\
& \times\left(\begin{array}{c}
\partial / \partial a_{\lambda} \\
\partial / \partial b_{\lambda} \\
\partial / \partial c_{\lambda}
\end{array}\right) .
\end{aligned}
$$

Similarly, the $\hat{L}_{\lambda_{1}}, \hat{L}_{\lambda_{2}}$, and $\hat{L}_{\lambda_{3}}$ are the space-fixed-type components of the $\boldsymbol{\delta}_{\boldsymbol{\lambda}}$-dependent internal angular momentum operator $\hat{\mathbf{L}}_{\lambda}$ defined by

$$
\begin{aligned}
\left(\begin{array}{c}
\hat{L}_{\lambda_{1}} \\
\hat{L}_{\lambda_{2}} \\
\hat{L}_{\lambda_{3}}
\end{array}\right) & \\
= & \frac{\hbar}{i}\left(\begin{array}{ccc}
-\cos \delta_{\lambda}^{(1)} \cot \delta_{\lambda}^{(2)} & -\sin \delta_{\lambda}^{(1)} & \cos \delta_{\lambda}^{(1)} \csc \delta_{\lambda}^{(2)} \\
-\sin \delta_{\lambda}^{(1)} \cot \delta_{\lambda}^{(2)} & \cos \delta_{\lambda}^{(1)} & \sin \delta_{\lambda}^{(1)} \csc \delta_{\lambda}^{(2)} \\
1 & 0 & 0
\end{array}\right) \\
& \times\left(\begin{array}{c}
\partial / \partial \delta_{\lambda}^{(1)} \\
\partial / \partial \delta_{\lambda}^{(2)} \\
\partial / \partial \delta_{\lambda}^{(3)}
\end{array}\right) .
\end{aligned}
$$

Under a $\lambda$ to $\nu$ change of Jacobi coordinates, the Jacobi matrix $\boldsymbol{\rho}_{\lambda}^{\text {sf }}$ changes according to the kinematic rotation,

$$
\boldsymbol{\rho}_{\nu}^{\mathrm{sf}}=\boldsymbol{\rho}_{\lambda}^{\mathrm{sf}} \mathbf{N}_{\lambda \nu},
$$

where $\mathbf{N}_{\lambda \nu}$ is a $3 \times 3$ proper orthogonal square matrix whose elements depend only on the masses of the atoms and on the clustering schemes $\lambda$ and $\nu$. The coordinates $\chi, \rho, \theta$, and $\phi$ are kinematic-rotation-invariant, as are the operators $\hat{T}_{\rho}(\rho)$, $\hat{\wedge}^{2}, \hat{K}^{2}, \hat{B}$, and $\hat{C}^{2}$. On the other hand, $\mathbf{R}\left(\mathbf{a}_{\lambda}\right), \mathbf{R}\left(\boldsymbol{\delta}_{\lambda}\right), \hat{\mathbf{J}}^{I_{\lambda}}$, and $\hat{\mathbf{L}}_{\lambda}$ transform according to

$$
\begin{aligned}
& \mathbf{R}\left(\mathbf{a}_{\nu}\right)=\mathbf{I}_{n_{\lambda \nu}^{(1)} n_{\lambda \nu}^{(3)} \mathbf{R}\left(\mathbf{a}_{\lambda}\right),} \\
& \mathbf{R}\left(\boldsymbol{\delta}_{\nu}\right)=\widetilde{\mathbf{N}}_{\lambda \nu} \mathbf{R}\left(\boldsymbol{\delta}_{\lambda}\right) \mathbf{I}_{n_{\lambda \nu}^{(1)} n_{\lambda \nu}^{(3)},} \\
& \hat{\mathbf{J}}^{I_{\nu}}=\mathbf{I}_{n_{\lambda \nu}^{(1)} n_{\lambda \nu}^{(3)}} \hat{\mathbf{J}}^{I_{\lambda}},
\end{aligned}
$$$$
\hat{\mathbf{L}}_{\nu}=\mathbf{I}_{n_{\lambda \nu}^{(1)} n_{\lambda \nu}^{(3)} \hat{\mathbf{L}}_{\lambda},},
$$

where

$$
\mathbf{I}_{n_{\lambda \nu}^{(1)} n_{\lambda \nu}^{(3)}}=\left(\begin{array}{ccc}
(-1)^{n_{\lambda \nu}^{(1)}} & 0 & 0 \\
0 & (-1)^{n_{\lambda \nu}^{(1)}+n_{\lambda \nu}^{(3)}} & 0 \\
0 & 0 & (-1)^{n_{\lambda \nu}^{(1)}}
\end{array}\right) .
$$

The quantities $n_{\lambda \nu}^{(1)}$ and $n_{\lambda \nu}^{(3)}$ are either 0 or 1 and depend on $\boldsymbol{\delta} \boldsymbol{\delta}$. As a result, either none or two of the components of $\hat{\mathbf{J}}^{I_{\nu}}$ and $\hat{\mathbf{L}}_{\lambda}$ change sign under kinematic rotations. Furthermore, the axes of the principal-axes-of-inertia frame $O x_{1}^{I_{\lambda}} x_{2}^{I_{\lambda}} x_{3}^{I_{\lambda}}$ are invariant under such rotations, and the senses of either none or two of them change. The hyperangular momentum operator of Eq. (2.16), and therefore the kinetic energy operator of Eq. (2.15), has singularities at the configurations for which at least two of the system's three principal moments of inertia are equal. This occurs for prolate and oblate symmetric top as well as for spherical top configurations. Unlike collinearly-dominated triatomic systems in which one singularity in the Hamiltonian corresponds to high energy regions of the potential energy surface, for tetra-atomic systems these singularities, in general, are not located in such regions. Therefore the wave function, which for low energies vanishes at that high-energy singularity of triatomic systems, does not at the singularities of tetraatomic systems. These 
singularities result in convergence difficulties for the most common quadrature or basis set expansion methods, including DVR methods. Littlejohn et al. did a detailed analysis ${ }^{19}$ of these difficulties. In the present paper, we develop a set of analytical basis functions which overcome this problem.

\section{HYPERSPHERICAL FUNCTIONS AND PRINCIPAL-AXES-OF-INERTIA HYPERSPHERICAL HARMONICS}

For tetra-atomic systems, the six operators $\hat{\wedge}^{2}, \hat{J}^{2}, \hat{J}_{z}^{\text {sf }}$, $\hat{L}^{2}, \hat{L}_{\lambda_{3}}^{\text {bf }}$, and $\hat{I}$ commute with each other. The first four have been defined in Sec. II. $\hat{L}_{\lambda_{3}}^{\text {bf }}$ is the third component of $\hat{\mathbf{L}}_{\lambda}^{\text {bf }}$ which by definition is the body-fixed-type operator,

$$
\hat{\mathbf{L}}_{\lambda}^{b f}=\mathbf{R}\left(\boldsymbol{\delta}_{\lambda}\right) \hat{\mathbf{L}}_{\lambda},
$$

where $\hat{\mathbf{L}}_{\lambda}$ has been defined in Eq. (2.26), and is explicitly given by

$$
\hat{L}_{\lambda_{3}}^{\mathrm{bf}}=\frac{\hbar}{i} \frac{\partial}{\partial \delta_{\lambda}^{(3)}} .
$$

Finally, $\hat{I}$ is the operator which inverts the system through its center-of-mass,

$$
\hat{I} \boldsymbol{\rho}_{\lambda}^{\mathrm{sf}}=-\boldsymbol{\rho}_{\lambda}^{\mathrm{sf}} .
$$

As has been shown previously, ${ }^{17} \hat{I}$ does not affect $\rho$ nor the $\boldsymbol{\Theta}_{\lambda}$ variables of Eq. (2.3), i.e.,

$$
\hat{I}\left(\rho, \boldsymbol{\Theta}_{\lambda}\right)=\left(\rho, \boldsymbol{\Theta}_{\lambda}\right),
$$

but changes $\chi=0,1$ to $\chi=1,0$ respectively, i.e.,

$$
\hat{I} \chi=\chi+1 \bmod 2 \text {. }
$$

Let $F^{\Pi n}{ }_{M_{J}}^{J}{ }_{L_{\lambda}}^{L}{ }_{d}^{D}\left(\chi, \boldsymbol{\Theta}_{\lambda}\right)$ be the simultaneous eigenfunctions of those six operators,

$$
\hat{\wedge}^{2} F^{\Pi n} \underset{M_{J} M_{L_{\lambda}}^{J}}{L} \underset{d}{D}\left(\chi, \boldsymbol{\Theta}_{\lambda}\right)=n(n+7) \hbar^{2} F^{\Pi n} \underset{M_{J} M_{L_{\lambda}} \underset{d}{L}}{D}\left(\chi, \boldsymbol{\Theta}_{\lambda}\right),
$$

$\hat{J}^{2} F^{\Pi n} \underset{M_{J} M_{L_{\lambda}}}{L} d \underset{d}{L}\left(\chi, \boldsymbol{\Theta}_{\lambda}\right)=J(J+1) \hbar^{2} F^{\Pi n} \underset{M_{J} M_{L_{\lambda}}}{L} \underset{d}{D}\left(\chi, \boldsymbol{\Theta}_{\lambda}\right)$,

$\hat{J}_{z}^{\mathrm{sf}} F^{\Pi n}{ }_{M_{J}{ }_{J} M_{L_{\lambda}}^{L}}^{L} \underset{d}{D}\left(\chi, \boldsymbol{\Theta}_{\lambda}\right)=M_{J} \hbar F^{\Pi n} \underset{M_{J}{ }_{M_{L_{\lambda}}}^{L}}{\stackrel{D}{d}}\left(\chi, \boldsymbol{\Theta}_{\lambda}\right)$,

$\hat{L}^{2} F^{\Pi n}{ }_{M_{J}{ }_{J} M_{L_{\lambda}}}^{L} \underset{d}{D}\left(\chi, \boldsymbol{\Theta}_{\lambda}\right)=L(L+1) \hbar^{2} F^{\Pi n} \underset{M_{J} M_{L_{\lambda}}^{J}}{\stackrel{L}{d}} \stackrel{D}{d}\left(\chi, \boldsymbol{\Theta}_{\lambda}\right)$,

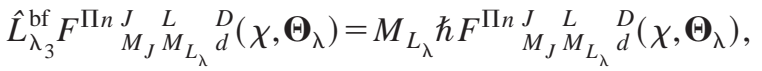

$\hat{I} F^{\Pi n} \underset{M_{J}{ }_{M_{L_{\lambda}}}^{J}}{\stackrel{D}{d}}\left(\chi, \boldsymbol{\Theta}_{\lambda}\right)=(-1)^{\Pi} F^{\Pi n} \underset{M_{J} M_{L_{\lambda}}^{J}}{\stackrel{D}{d}}\left(\chi, \boldsymbol{\Theta}_{\lambda}\right)$.

These $F$ functions are furthermore required to be regular at the poles of $\hat{\wedge}^{2}$ expressed by Eqs. (2.16) and (2.18)-(2.24). The quantum numbers $n, J, M_{J}, L, M_{L_{\lambda}}$, and $\Pi$ appearing in these expression are all integers, satisfying the constraints

$$
\begin{aligned}
& n \geqslant 0 \quad 0 \leqslant J, L \leqslant n, \\
& -J \leqslant M_{J} \leqslant J, \quad-L \leqslant M_{L_{\lambda}} \leqslant L,
\end{aligned}
$$

$$
\Pi=0,1 .
$$

The five operators $\hat{\wedge}^{2}, \hat{J}^{2}, \hat{J}_{z}^{\text {sf }}, \hat{L}^{2}$, and $\hat{I}$ are all independent of the choice of arrangement channel coordinates $\lambda$ (Ref. 17) and, therefore, so as are the corresponding quantum numbers. However, from Eqs. (3.1), (2.26), (2.29), and (2.31) one gets

$$
\hat{\mathbf{L}}_{\nu}^{\mathrm{bf}}=\widetilde{\mathbf{N}}_{\lambda \nu} \hat{\mathbf{L}}_{\lambda}^{\mathrm{bf}}
$$

As a result $\hat{\mathbf{L}}_{\lambda}^{\text {bf }}$, and therefore $\hat{L}_{\lambda_{3}}^{\text {bf }}$, are not kinematicrotation-invariant, which justifies the subscript $\lambda$ in the quantum number $M_{L_{\lambda}}$. The positive integer superscript $D$ is used to label the number of linearly-independent $F$ functions having the same set of quantum numbers $n, J, M_{J}, L$, and $M_{L_{\lambda}}$. This $D$ degeneracy stems from the fact that the system of four free particles in a center-of-mass frame has eight angular degrees of freedom, as indicated by Eq. (2.3) (and as a result has eight simultaneously knowable angular constants of the motion), but $F$ has been required to be an eigenfunction of only five operators in these angular variables. It is shown in Sec. V B that $D$ depends on the quantum numbers $n, J$, and $L$ (but not on $M_{J}$ or $M_{L_{\lambda}}$ ). The positive integer subscript $d$, which ranges from 1 to $D$, designates which of the $D F$ functions is being considered.

As a result of Eqs. (3.6)-(3.10) and of the fact that none of the corresponding five operators contains $\chi$, the general solution of those equations plus Eq. (3.11) can be written as

$$
\begin{aligned}
F^{\Pi n} \underset{M_{J} M_{L_{\lambda}}}{L} \underset{d}{D}\left(\chi, \boldsymbol{\Theta}_{\lambda}\right) \\
=N^{n J L}{ }_{d}^{D} f^{\Pi}(\chi) \sum_{\Omega_{J_{\lambda}}=-J}^{J} \sum_{\Omega_{L_{\lambda}}=-L}^{L} D_{M_{J} \Omega_{J_{\lambda}}}^{J}\left(\mathbf{a}_{\lambda}\right) \\
\quad \times D_{\Omega_{L_{\lambda}}^{L} M_{L_{\lambda}}}^{L}\left(\boldsymbol{\delta}_{\lambda}\right) G^{n J}{ }_{\Omega_{J_{\lambda}}}^{L} \Omega_{L_{\lambda}}{ }_{d}^{D}(\theta, \phi) .
\end{aligned}
$$

The presence of the Wigner rotation functions $D_{M_{J} \Omega_{J_{\lambda}}}^{J}\left(\mathbf{a}_{\lambda}\right)$ and $D_{\Omega_{L_{\lambda}} M_{L_{\lambda}}}^{L}\left(\boldsymbol{\delta}_{\lambda}\right)$ (Ref. 25) guarantees that Eq. (3.16) will satisfy Eqs. (3.7)-(3.10). As shown in Appendix A, replacement into Eq. (3.6) results in a set of partial differential equations for the functions $G$ which contain neither $M_{J}$ nor $M_{L_{\lambda}}$ and therefore the $G$ are independent of these quantum numbers. This independence is a consequence of the fact that $\hat{\wedge}^{2}$ is invariant under both space and kinematic rotations. The degeneracy $D(n, J, L)$ also represents the number of linearlyindependent sets of functions $\left\{G^{n}{ }_{\Omega_{J_{\lambda}}} \Omega_{L_{\lambda}}^{L}{ }^{D}\right\}$, each set spanned, as discussed in Sec. V B, by the quantum numbers $\Omega_{J_{\lambda}}$ and $\Omega_{L_{\lambda}}$. The $N$ in the right-hand-side of Eq. (3.16) is a normalization constant that will be discussed in Sec. V. The chirality function $f^{\Pi}(\chi)$ is needed to make Eq. (3.16) satisfy Eq. (3.11). As shown in Appendix B, that function can be chosen to be

$$
f^{\Pi}(\chi)=(-1)^{n \chi}=(-1)^{\Pi \chi}
$$

and we can rewrite Eq. (3.16) as 


$$
\begin{aligned}
& F^{\Pi n_{\Pi}}{ }_{M_{J}}^{J} \underset{M_{L_{\lambda}}}{L} \stackrel{D}{d}\left(\chi, \boldsymbol{\Theta}_{\lambda}\right) \\
& =N^{n} \Pi^{J L} \underset{d}{D}(-1)^{\Pi \chi} \sum_{\Omega_{J_{\lambda}}=-J}^{J} \sum_{\Omega_{L_{\lambda}}=-L}^{L} D_{M_{J} \Omega_{J_{\lambda}}}^{J}\left(\mathbf{a}_{\lambda}\right) \\
& \times D_{\Omega_{L_{\lambda}} M_{L_{\lambda}}}^{L}\left(\boldsymbol{\delta}_{\lambda}\right) G^{n} \prod_{\Omega_{J_{\lambda}}}^{J} \stackrel{\Omega_{L_{\lambda}}}{L} \quad \stackrel{D}{d}(\theta, \phi) .
\end{aligned}
$$

As also shown in that appendix, $n_{\Pi}$ is zero or an even positive integer for $\Pi=0$ and an odd positive integer for $\Pi=1$. The corresponding $F$ functions are, respectively, symmetric and antisymmetric with respect to inversion through the system's center-of-mass, as required by Eq. (3.11). If we restrict ourselves to single electronically-adiabatic states of the tetraatomic system being considered, the potential energy function $V\left(\rho, \theta, \phi, \boldsymbol{\delta}_{\lambda}\right)$ which describes the interaction between those atoms is invariant (i.e., symmetric) under such inversion, and matrix elements of $V$ between $F$ functions of different parity vanish. Since the range of the $\boldsymbol{\delta}_{\lambda}$ angles is given by Eq. (2.8), Eq. (3.16) is also restricted to that range. However, the $D_{\Omega_{L_{\lambda} M_{L_{\lambda}}}^{L}}\left(\boldsymbol{\delta}_{\lambda}\right)$ functions are only orthonormal over the range

$$
0 \leqslant \delta_{\lambda}^{(1)}, \delta_{\lambda}^{(3)}<2 \pi, \quad 0 \leqslant \delta_{\lambda}^{(2)} \leqslant \pi
$$

Therefore, it is desirable to permit Eq. (3.18) to be valid over this extended range. This can be accomplished by noticing that the four sets of ROHC

$$
\left(\boldsymbol{\gamma}_{\lambda_{i}}\right)_{i}=\left(\chi, \rho, \boldsymbol{\Theta}_{\lambda_{i}}\right), \quad i=0,1,2,3
$$

defined by

$$
\begin{aligned}
\boldsymbol{\Theta}_{\lambda_{0}}= & \left(a_{\lambda}, b_{\lambda}, c_{\lambda}, \theta, \phi, \delta_{\lambda}^{(1)}, \delta_{\lambda}^{(2)}, \delta_{\lambda}^{(3)}\right), \\
\boldsymbol{\Theta}_{\lambda_{1}}= & \left(\left(\pi+a_{\lambda}\right) \bmod 2 \pi, \pi-b_{\lambda},\right. \\
& \left(\pi-c_{\lambda}\right) \bmod 2 \pi, \theta, \phi,\left(\pi-\delta_{\lambda}^{(1)}\right) \bmod 2 \pi, \pi \\
& \left.-\delta_{\lambda}^{(2)},\left(\pi+\delta_{\lambda}^{(3)}\right) \bmod 2 \pi\right), \\
\boldsymbol{\Theta}_{\lambda_{2}}= & \left(\left(\pi+a_{\lambda}\right) \bmod 2 \pi, \pi-b_{\lambda},\right. \\
& \left(2 \pi-c_{\lambda}\right) \bmod 2 \pi, \theta, \phi,\left(\pi-\delta_{\lambda}^{(1)}\right) \bmod 2 \pi, \pi \\
& \left.-\delta_{\lambda}^{(2)},\left(\pi+\delta_{\lambda}^{(3)}\right) \bmod 2 \pi\right)
\end{aligned}
$$

and

$$
\begin{array}{r}
\boldsymbol{\Theta}_{\lambda_{3}}=\left(a_{\lambda}, b_{\lambda},\left(\pi+c_{\lambda}\right) \bmod 2 \pi, \theta, \phi,\right. \\
\left.\left(\pi+\delta_{\lambda}^{(1)}\right) \bmod 2 \pi, \delta_{\lambda}^{(2)}, \delta_{\lambda}^{(3)}\right),
\end{array}
$$

with the $\boldsymbol{\delta}_{\boldsymbol{\lambda}}$ in the ranges defined by Eq. (3.19), yield the same Jacobi matrix of Eq. (2.1), i.e., correspond to the same configuration of the system. As a result, the system's wave function, in the absence of a conical intersection between the electronically-adiabatic potential energy function being considered and a neighboring one (i.e., in the absence of a geometric phase effect ${ }^{26}$ ), should have the same value at these four sets of ROHC. We therefore impose the same condition on Eq. (3.16). Replacement of Eqs. (3.21)-(3.24) into Eq. (3.16) and use of this condition leads to the two relations,

$$
\begin{aligned}
& G_{\Omega_{J_{\lambda}} \Omega_{L_{\lambda}}^{J}}^{L} \stackrel{D}{d}(\theta, \phi)=0 \text { for } \Omega_{J_{\lambda}}+\Omega_{L_{\lambda}} \text { odd, } \\
& G^{n} \underset{-\Omega_{J_{\lambda}}-\Omega_{L_{\lambda}} \underset{d}{D}}{\stackrel{D}{d}}(\theta, \phi)=(-1)^{(J+L)} G^{n} \underset{\Omega_{J_{\lambda}}^{J}}{\stackrel{L}{\Omega_{L_{\lambda}}}} \underset{d}{D}(\theta, \phi) .
\end{aligned}
$$

Since the $G$ are independent of $\boldsymbol{\delta}_{\lambda}$, these relations should be imposed regardless of whether the ranges defined by Eq. (2.8) or Eq. (3.19) are being considered, and therefore Eqs. (3.16) and (3.18) are valid in either of these two ranges. As a result of Eq. (3.25), the sum in the right-hand side of those expressions are restricted to values of $\Omega_{J_{\lambda}}$ and $\Omega_{L_{\lambda}}$ for which $\Omega_{J_{\lambda}}+\Omega_{L_{\lambda}}$ is even. Such a restriction will be implied from now on. It should be stressed, however, that Eqs. (3.25) and (3.26) are valid only in the absence of a geometric phase. If such a phase is present, a different approach is required, and the $F$ and $G$ of this paper are not applicable. Finally, in addition to Eqs. (3.25) and (3.26), the following symmetry property, derived in Appendix A as Eq. (A35), is valid:

$$
G^{n}{ }_{\Omega_{J_{\lambda}}^{J}} \Omega_{L_{\lambda}}^{L} \stackrel{D}{d}(\theta, \phi)=G^{n} \stackrel{L}{\Omega_{J_{\lambda}}} \underset{\Omega_{L_{\lambda}}}{J} \quad \underset{d}{D}(\theta, \phi)
$$

Equations (3.25)-(3.27) greatly decrease the number of $G$ functions that must be independently evaluated. The $F$ functions given by Eqs. (3.16) or (3.18) are called eight-angle principal-axes-of-inertia hyperspherical harmonics, and the $G$ functions in those equations are called two-angle principal-axes-of-inertia hyperspherical harmonics, or simply $F$ hyperspherical harmonics or functions and $G$ hyperspherical harmonics or functions respectively. The $F$ functions, which depend on the eight hyperangles $\boldsymbol{\Theta}_{\lambda}$ and on the chirality coordinate $\chi$, constitute an appropriate complete linearlyindependent basis set in these variables, in terms of which the local hyperspherical surface functions (LHSF), defined in the first paragraph of Sec. VII, may be expanded. The coefficients of this expansion will depend only on the hyperradius $\rho$. An important property of the $F$ functions is that they behave regularly at the poles of the kinetic energy operator $\hat{T}$ of Eq. (2.15) and therefore of the system's Hamiltonian. They are, in addition, $\rho$-independent. If they could be obtained analytically, they would constitute a very useful basis set. In the rest of this paper we show how indeed we can obtain analytical expression for the $G$ functions and therefore for the $F$ functions. It should be noted that the matrix representation of $\hat{T}$ in the $F$ basis set is completely diagonal. All the Coriolis couplings involving $\Omega_{J_{\lambda}}$ and $\Omega_{L_{\lambda}}$ are automatically included in the evaluation of the $G$ functions. The only matrix elements that must be evaluated numerically are those of the potential energy function. It should also be noted that if some of the system's atoms are equal, it is possible to define modified $F$ functions that transform according to the irreducible representations of the permutation group of iden- 
tical atoms. This, will entail a modification of the $D_{\Omega_{L_{\lambda}} M_{L_{\lambda}}}^{L}\left(\boldsymbol{\delta}_{\lambda}\right)$ functions in Eqs. (3.16) or (3.18), but not of the associated $G$ functions.

\section{ANALYTICAL DERIVATION OF TWO-ANGLE PRINCIPAL-AXES-OF-INERTIA HYPERSPHERICAL HARMONICS $G$}

\section{A. General considerations}

Since the kinetic energy operator $\hat{T}$ of Eq. (2.15) is the Hamiltonain of four noninteracting particles (for which $V$ $=0)$, its eigenfunctions can be obtained analytically, as follows. Let $r_{\lambda}^{i}, \theta_{\lambda}^{(i)}, \phi_{\lambda}^{(i)}(i=1,2,3)$ be the space-fixed polar coordinates of the mass-scaled $\lambda$-arrangement channel Jacobi coordinates $\mathbf{r}_{\lambda}^{(i)}$ introduced in Sec. II. The eigenfunctions of $\hat{T}$ can be expressed as products of the three ordinary spherical harmonics of $\theta_{\lambda}^{(i)}, \phi_{\lambda}^{(i)}$ times a function of $r_{\lambda}^{(1)}, r_{\lambda}^{(2)}$, $r_{\lambda}^{(3)}$. These latter three variables can be transformed into the hyper-radius $\rho$ and two hyperangles $\eta_{\lambda_{1}}$ and $\eta_{\lambda_{2}}$ defined by the relations,

$$
\begin{aligned}
& r_{\lambda}^{(1)}=\rho \sin \eta_{\lambda_{2}} \sin \eta_{\lambda_{1}}, \\
& r_{\lambda}^{(2)}=\rho \sin \eta_{\lambda_{2}} \cos \eta_{\lambda_{1}}, \\
& r_{\lambda}^{(3)}=\rho \cos \eta_{\lambda_{2}} .
\end{aligned}
$$

At a constant $\rho$, the partial differential equations in $r_{\lambda}^{(1)}$, $r_{\lambda}^{(2)}$, and $r_{\lambda}^{(3)}$ are transformed into two separate ordinary differential equations, one for $\eta_{\lambda_{1}}$ and another for $\eta_{\lambda_{2}}$, whose solutions are known hypergeometric functions of the square of the cosines of these two angles. In this way the eigenfunctions of $\hat{\wedge}^{2}$, for a quantum number $n$, become known functions of the eight angles $\theta_{\lambda}^{(i)}, \phi_{\lambda}^{(i)}(i=1,2,3), \eta_{\lambda_{1}}$ and $\eta_{\lambda_{2}}$. They are however not eigenfunctions of the remaining operators of Eqs. (3.7)-(3.11). One can however transform them into functions of the eight hyperangles $\boldsymbol{\Theta}_{\lambda}$ and expand them in the basis set $F$. This procedure will furnish the $G$ functions analytically. This kind of approach, with slightly different hyperangular coordinates, was used by Zickendraht $^{20}$ to obtain all $G$ for $n=1$ and 2. We repeated it for our ROHC and obtained equivalent results. Proceeding to the $n=3$ and 4 cases, the corresponding $G$ were obtained, but the amount of analytical effort increased significantly. The amount of time required to perform the associated algebra manually for $n=4$ and to check the results was about 30 $\mathrm{h}$, and yielded the $450 \mathrm{G}$ functions predicted. Because of Eqs. (3.25)-(3.27), only 93 of those had to be calculated. For a general $n$, the number of $G$ functions for a fixed $n$ [including the ones that vanish due to Eq. (3.25)] is given by Eq. (5.11). For the $\mathrm{H}_{2}+\mathrm{OH} \rightarrow \mathrm{H}_{2} \mathrm{O}+\mathrm{H}$ reaction, at a relative translational energy of $0.7 \mathrm{eV}$ between ground state reagents, values of $J$ up to 30 may be required to obtain state-to-state differential cross sections of benchmark quality. Since, from Eq. (3.12), $n \geqslant J, G$ functions up to at least $n=30$ may be needed in these calculations. For this value of $n$, Eq. (5.11) furnishes approximately 18.6 million $G$ functions, of which 2.3 million have to be evaluated. This manual algebra approach cannot be used to obtain such large number of functions. What is required instead is some automatic analytical procedure that can be implemented on a computer. The one just described is too complicated to be done by any existing computer algebra program. An alternative that could be feasible would be one based on a recursion relation between the $G$ function for a given $n$ to those for $n+1$. If such a relation were simple enough, it might be possible to use a computer algebra program to generate the large number of needed analytical $G$ functions. In the rest of this section we derive such a recursion relation. It is based on the theory of harmonic polynomial $^{24}$ and the use of complex coordinates. ${ }^{20}$

\section{B. Complex coordinates and the corresponding Hamiltonian}

Complex coordinates were previously used in connection with this problem by Zickendraht. ${ }^{20} \mathrm{He}$ introduced them however in an ad hoc manner. The approach described below is slightly different and gives a rationale for their definition. Equation (2.4) describes the defining relation between the ROHC and the Jacobi mass-scaled space-fixed cartesian coordinates for the $\lambda$-arrangement channel clustering scheme. By expressing the elements of the $\mathbf{R}$ matrices in its righthand side in terms of Wigner rotation functions, an intermediate set of complex coordinates suggests itself naturally. Indeed, with the help of the explicit expression of the Wigner rotation matrix $\mathbf{D}^{1},{ }^{25}$ it is straightforward to derive the expression

$$
\mathbf{R}=\left(\begin{array}{ccc}
\frac{1}{2}\left(D_{11}^{1}-D_{1-1}^{1}-D_{-11}^{1}+D_{-1-1}^{1}\right) & \frac{-i}{2}\left(D_{11}^{1}-D_{1-1}^{1}+D_{-11}^{1}-D_{-1-1}^{1}\right) & \frac{1}{\sqrt{2}}\left(D_{01}^{1}-D_{0-1}^{1}\right) \\
\frac{i}{2}\left(D_{11}^{1}+D_{1-1}^{1}-D_{-11}^{1}-D_{-1-1}^{1}\right) & \frac{1}{2}\left(D_{11}^{1}+D_{1-1}^{1}+D_{-11}^{1}+D_{-1-1}^{1}\right) & \frac{i}{\sqrt{2}}\left(D_{01}^{1}+D_{0-1}^{1}\right) \\
\frac{1}{\sqrt{2}}\left(D_{10}^{1}-D_{-10}^{1}\right) & \frac{-i}{\sqrt{2}}\left(D_{10}^{1}+D_{-10}^{1}\right) & D_{00}^{1}
\end{array}\right),
$$


where $\mathbf{R}$ and the $D_{k j}^{1}(k, j=-1,0,1)$ are evaluated at the same set of Euler angles. Replacement of Eq. (4.4) into Eq. (2.4) leads to the nine relations,

$$
\begin{aligned}
& x_{\lambda 1}^{(1)}=\frac{1}{2}\left(t_{\lambda 1}^{1}-t_{\lambda-1}^{1}-t_{\lambda}^{-1}+t_{\lambda-1}^{-1}\right), \\
& x_{\lambda 2}^{(1)}=\frac{1}{2 i}\left(t_{\lambda 1}^{1}-t_{\lambda-1}^{1}+t_{\lambda}^{-1}-t_{\lambda-1}^{-1}\right), \\
& x_{\lambda 3}^{(1)}=\frac{1}{\sqrt{2}}\left(t_{\lambda 1}^{0}-t_{\lambda-1}{ }^{0}\right), \\
& x_{\lambda 1}^{(2)}=-\frac{1}{2 i}\left(t_{\lambda 1}^{1}+t_{\lambda-1}^{1}-t_{\lambda}^{-1}{ }_{1}^{1}-t_{\lambda-1}^{-1}\right), \\
& x_{\lambda 2}^{(2)}=\frac{1}{2}\left(t_{\lambda 1}^{1}+t_{\lambda-1}{ }^{1}+t_{\lambda}^{-1}+t_{\lambda-1}^{-1}\right), \\
& x_{\lambda 3}^{(2)}=-\frac{1}{i \sqrt{2}}\left(t_{\lambda 1}^{0}+t_{\lambda-1}^{0}\right), \\
& x_{\lambda 1}^{(3)}=\frac{1}{\sqrt{2}}\left(t_{\lambda 0}^{1}-t_{\lambda}^{-1} 0\right), \\
& x_{\lambda 2}^{(3)}=\frac{1}{i \sqrt{2}}\left(t_{\lambda 0}^{1}+t_{\lambda}^{-1} 0\right) \text {, } \\
& x_{\lambda 3}{ }^{(3)}=t_{\lambda 0}{ }^{0} \text {, }
\end{aligned}
$$

where the complex quantities $t_{\lambda j}^{k}\left(\chi, \rho, \boldsymbol{\Theta}_{\lambda}\right)$ are defined by

$$
t_{\lambda j}^{k}=\rho(-1)^{\chi} \sum_{p, q=-1}^{1} D_{k p}^{1}\left(\mathbf{a}_{\lambda}\right) D_{q j}^{1}\left(\boldsymbol{\delta}_{\lambda}\right) \bar{t}_{p}^{q}(\theta, \phi),
$$

with

$$
\begin{aligned}
& \bar{t}_{1}^{1}=\bar{t}_{-1}^{-1}=\frac{1}{2}\left[N_{11}(\theta, \phi)+N_{22}(\theta, \phi)\right] \equiv x, \\
& \bar{t}_{-1}^{1}=\bar{t}_{1}^{-1}=\frac{1}{2}\left[N_{11}(\theta, \phi)-N_{22}(\theta, \phi)\right] \equiv y, \\
& \bar{t}_{0}^{0}=N_{33}(\theta, \phi) \equiv z, \\
& \bar{t}_{1}^{0}=\bar{t}_{-1}^{0}=\bar{t}_{0}^{1}=\bar{t}_{0}^{-1}=0 .
\end{aligned}
$$

In view of Eq. (4.18), the sum in Eq. (4.14) is limited to values of $p+q$ equal to $-2,0$, and 2 . We can consider the complex $t_{\lambda k}^{j}$ as midway variables between the Cartesian coordinates $x_{\lambda j}{ }^{(i)}(i, j=1,2,3)$ and the ROHC $\chi, \rho, \boldsymbol{\Theta}_{\lambda}$. The system's Laplacian in these variables, as shown in Appendix $\mathrm{C}$, is given by

$$
\nabla^{2}=\sum_{j, k=-1}^{1}(-1)^{j+k} \frac{\partial^{2}}{\partial t_{\lambda j}^{k} \partial t_{\lambda-j}^{-k}} .
$$

This is a particularly simple expression and well permit us, as seen in Sec. IV C, to derive a recursion relation between $F$ and $G$ functions for hyperangular momentum quantum numbers $n$ and $n+1$.

\section{Recursion relation for hyperspherical harmonics}

In this section we derive recursion relations for the $F$ and $G$ functions associated with consecutive values of the hyperangular momentum quantum number $n$. To do this, we make use of the properties of harmonic polynomials. ${ }^{24}$ These properties for $m$-dimensional space are summarized in Appendix D for convenience. We will now set $m=9$ (for tetraatomic systems) and from here on omit this index. Let $f^{n}\left(\mathbf{x}_{\lambda}\right)$ be an arbitrary homogeneous polynomial of degree $n$ in the nine real variables $\mathbf{x}_{\lambda} \equiv\left\{x_{\lambda_{l}}^{(i)}, \quad i, l=1,2,3\right\}$ given by Eq. (D1). The associated function $h^{n}\left(\mathbf{x}_{\lambda}\right)$ defined by Eq. (D5) is therefore a harmonic polynomial satisfying the ninedimensional Laplace equation,

$$
\nabla^{2} h^{n}\left(\mathbf{x}_{\lambda}\right)=0 .
$$

Let us define a new set of functions $f_{j k}^{n+1}\left(\mathbf{x}_{\lambda}\right)$ by

$$
f_{j k}^{n+1}\left(\mathbf{x}_{\lambda}\right)=t_{\lambda j}^{k}\left(\mathbf{x}_{\lambda}\right) h^{n}\left(\mathbf{x}_{\lambda}\right),
$$

where the $t_{\lambda j}{ }^{k}\left(\mathbf{x}_{\lambda}\right)$ are given by Eqs. (C5)-(C13). Since these $t_{\lambda j}{ }^{k}$ are homogeneous polynomials of the first degree in the $\mathbf{x}_{\lambda}$ components, the $f_{j k}^{n+1}\left(\mathbf{x}_{\lambda}\right)$ are homogeneous polynomials of degree $n+1$ in those variables. As a result of Eqs. (4.19) and (4.20), the following property can be easily derived:

$$
\nabla^{2 i} f_{j k}^{n+1}\left(\mathbf{x}_{\lambda}\right)=\left\{\begin{array}{l}
2(-1)^{j+k} \partial h^{n} / \partial t_{\lambda-j}^{-k} \\
0 \quad \text { for } i>1 .
\end{array} \text { for } i=1\right.
$$

It should be noted that the symbol $\partial h^{n} / \partial t_{\lambda-j}^{-k}$ implies that although $h^{n}$ is a harmonic polynomial in the variables $x_{\lambda_{l}}^{(i)}$ $(i, l=1,2,3)$, it can also be considered, with the help of Eqs. (C5)-(C13) to be a harmonic polynomial in the variables $t_{\lambda j}{ }_{j}$ $(j, k=-1,0,1)$.

Replacing Eq. (4.22) into the $n+1$ counterpart of Eq. (D5) we get

$$
h_{j k}^{n+1}=t_{\lambda j}{ }^{k} h^{n}-\frac{\rho^{2}}{2(2 n+7)} \nabla^{2}\left(t_{\lambda j}{ }^{k} h^{n}\right) .
$$

This is a recursion relation between the harmonic polynomial $h^{n}$ and each of the nine harmonic polynomials $h_{j k}^{n+1}(j, k=$ $-1,0,1)$ all of which are of degree $n+1$. Let us consider the

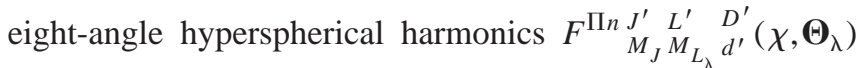
given by Eqs. (3.16) and (3.17) (with $J, L, D$, and $d$ replaced by $J^{\prime}, L^{\prime}, D^{\prime}$, and $d^{\prime}$, respectively) and where $\Pi$ is zero (one) for $n$ even (odd). Since it satisfies Eq. (3.6), we conclude from Eqs. (D4) and (D10) that the function $h^{\Pi n} \underset{M_{J} M_{L_{\lambda}}^{L^{\prime}}}{L^{\prime}} d^{D^{\prime}}\left(\rho, \chi, \boldsymbol{\Theta}_{\lambda}\right)$ defined by

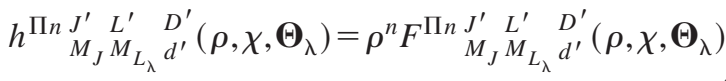

is a solution of the Laplace Eq. (4.20) and is therefore a harmonic polynomial of degree $n$ in the variables $x_{\lambda_{l}}^{(i)}$ or equivalently, the $t_{\lambda j}{ }^{k}$ to which the $\operatorname{ROHC} \rho, \chi, \boldsymbol{\Theta}_{\lambda}$ are related. Let us use this choice of $h^{n}$ in the right-hand side of Eq. (4.21). With the help of Eqs. (4.14), (3.16), and (3.17) and of the multiplication properties of the Wigner rotation functions, ${ }^{25}$ we get 


$$
\begin{aligned}
f_{j k}^{n+1}= & \sum_{J=\left|J^{\prime}-1\right|}^{J^{\prime}+1} \sum_{J=\left|L^{\prime}-1\right|}^{L^{\prime}+1} C\left(J^{\prime} 1 J ; M_{J} k M_{J}+k\right) \\
& \times C\left(L^{\prime} 1 L ; M_{L_{\lambda}} j M_{L_{\lambda}}+j\right) \\
& \times\left[\mathcal{F}^{n+1}{ }_{M_{J}+k}^{L} M_{L_{\lambda}}+j\right]_{J^{\prime} L^{\prime} d^{\prime}}
\end{aligned}
$$

$$
\begin{aligned}
{\left[\bar{F}^{n+1} \underset{M_{J}+k \stackrel{L}{M_{L_{\lambda}}}+j}{J}\right]_{J^{\prime} L^{\prime} d^{\prime}}=} & \frac{1}{\rho^{n+1}}\left[1-\frac{\rho^{2}}{2(2 n+7)} \nabla^{2}\right] \\
& \times\left\{\rho^{n+1}\left[\mathcal{F}^{n+1} \underset{M_{J}+k \stackrel{L}{M_{L_{\lambda}}}+j}{ }+\right]_{J^{\prime} L^{\prime} d^{\prime}}\right\},
\end{aligned}
$$

where

$$
\begin{aligned}
& {\left[\mathcal{F}^{n+1} \underset{M_{J}+k M_{L_{\lambda}}+j}{L}\right]_{J^{\prime} L^{\prime} d^{\prime}} } \\
&=(-1)^{(n+1) \chi} \sum_{\Omega_{J_{\lambda}}=-J}^{J} \sum_{\Omega_{L_{\lambda}}=-L}^{L} \\
& \times D_{M_{J}+k \Omega_{J_{\lambda}}^{J}}\left(\mathbf{a}_{\lambda}\right) D_{\Omega_{L_{\lambda}} M_{L_{\lambda}}+j^{\prime}}^{L}\left(\boldsymbol{\delta}_{\lambda}\right) \\
& \times\left[\mathcal{G}^{n+1}{ }_{\Omega_{J_{\lambda}} \Omega_{L_{\lambda}}^{L}}^{L}(\theta, \phi)\right]_{J^{\prime} L^{\prime} d^{\prime}}
\end{aligned}
$$

and

$$
\begin{aligned}
& {\left[\mathcal{G}^{n+1} \underset{\Omega_{J_{\lambda}}}{J} \Omega_{L_{\lambda}}^{L}(\theta, \phi)\right]_{J^{\prime} L^{\prime} d^{\prime}}} \\
& =\sum_{p, q=-1}^{1} C\left(J^{\prime} 1 J ; \Omega_{J_{\lambda}}-p p \Omega_{J_{\lambda}}\right) \\
& \times C\left(L^{\prime} 1 L ; \Omega_{L_{\lambda}}-q q \Omega_{L_{\lambda}}\right) \bar{t}_{p}^{q} G^{n}{ }_{\Omega_{J_{\lambda}}-p}^{J^{\prime}} \Omega_{L_{\lambda}}^{L^{\prime}}-q d^{\prime} D^{\prime} .
\end{aligned}
$$

The $C$ in the last three equations are Clebsch-Gordan coefficients, in Rose's notation. ${ }^{27}$ In addition, the $J^{\prime}$ and $L^{\prime}$ that appear as subscripts in those equations are related to $J$ and $L$ by the triangle inequalities,

$$
\left|J^{\prime}-1\right| \leqslant J \leqslant J^{\prime}+1, \quad\left|L^{\prime}-1\right| \leqslant L \leqslant L^{\prime}+1 .
$$

The subscript $d^{\prime}$ in the right-hand side of Eq. (4.25) and in both sides of Eqs. (4.26) and (4.27) indicates that each set of $G^{n}$ functions with subscript $d^{\prime}$ generates a corresponding set of $\left[\mathcal{G}^{n+1}\right]_{J^{\prime} L^{\prime} d^{\prime}}$ and of $\left[\mathcal{F}^{n+1}\right]_{J^{\prime} L^{\prime} d^{\prime}}$ functions according to those equations. These $\mathcal{G}^{n+1}$ and $\mathcal{F}^{n+1}$ functions do not have a $D$ superscript because they are not $G$ and $F$ functions respectively. Due to the Wigner rotation functions in the right-hand side of Eq. (4.26), we see that $\left[\mathcal{F}^{n+1}{ }_{M_{J}+k}^{J}{ }_{M_{L_{\lambda}}}^{L}+j\right]_{J^{\prime} L^{\prime} d^{\prime}}$ is a simultaneous eigenfunction of $\hat{J}^{2}, \hat{J}_{z}^{\text {sf }}, \hat{L}^{2}$, and $\hat{L}_{\lambda_{3}}^{\text {bf }}$ [the latter defined by Eq. (3.2)] with eigenvalues $J(J+1) \hbar^{2},\left(M_{J}+k\right) \hbar, L(L+1) \hbar^{2}$, and $\left(M_{L_{\lambda}}\right.$ $+j) \hbar$, respectively. Inserting Eq. (4.25) into Eq. (4.23), and adding to $h_{j k}^{n+1}$ appropriate indices that appear in the righthand side of Eq. (4.25), we obtain

$$
\begin{aligned}
h_{j k}^{n+1} M_{J}+k M_{L_{\lambda}+j}^{L^{\prime}} & L^{\prime} \\
= & \rho^{n+1} \sum_{J=\left|J^{\prime}-1\right|}^{J^{\prime}+1} \sum_{L=\left|L^{\prime}-1\right|}^{L^{\prime}+1} C\left(J^{\prime} 1 J ; M_{J} k M_{J}+k\right) \\
& \times C\left(L^{\prime} 1 L ; M_{L_{\lambda}} j M_{L_{\lambda}}+j\right)\left[\bar{F}^{n+1}{ }_{M_{J}+k M_{L_{\lambda}}+j}^{L}\right]_{J^{\prime} L^{\prime} d^{\prime}},
\end{aligned}
$$

where and is independent of $\rho$. In the left-hand side of Eq. (4.29), let us allow $j$ and $k$ to assume all their possible values, while at the same time varying $M_{J}$ and $M_{L_{\lambda}}$ so as to maintain $M_{J}+k$ and $M_{L_{\lambda}}+j$ constant. This will generate, for $J^{\prime}>0$ and $L^{\prime}>0$, nine $h_{j k}^{n+1}{ }_{M_{J}^{\prime}+k}^{J^{\prime}} M_{L_{\lambda}+j}^{L^{\prime}} d^{\prime}$. Each of them will be a different linear combination of the same set of nine functions $\left[\bar{F}^{n+1}{ }_{M_{J}+k M_{L_{\lambda}}}^{L}+j\right]_{J^{\prime} L^{\prime} d^{\prime}}$, in which $J=J^{\prime}-1, J^{\prime}, J^{\prime}+1$ and $L=L^{\prime}-1, L^{\prime}, L^{\prime}+1$. (The particular case $J^{\prime}=0$ and/or $L^{\prime}$ $=0$ can be considered similarly.) These relations can be inverted, so as to express each of the $\left[\bar{F}^{n+1}{ }_{M_{J}+k M_{L_{\lambda}}}^{L}{ }_{j}\right]_{J^{\prime} L^{\prime} d^{\prime}}$ as different linear combinations of the nine $h_{j k}^{n+1}{ }_{M_{J}^{\prime}+k}^{L^{\prime}} M_{L_{\lambda}+j}^{d^{\prime}}(\theta, \phi) / \rho^{n+1}$. Since the $h_{j k}^{n+1}$ are all harfrom Eq. (D18) that each of these $\bar{F}^{n+1}$ are eigenfunctions of $\hat{\wedge}^{2}$ with hyperangular momentum quantum number $n+1$. In addition, since $\nabla^{2}$ commutes with $\hat{J}^{2}, \hat{J}_{z}^{\text {sf }}, \hat{L}^{2}$, and $\hat{L}_{\lambda_{3}}^{\text {bf }}$, and since, as pointed out after Eq. (4.28), the $\left[\mathcal{F}^{n+1}{ }_{M_{J}+k}^{J} M_{L_{\lambda}}^{L}+j\right]_{J^{\prime} L^{\prime} d^{\prime}}$ are eigenfunctions of these operators, we conclude, from Eq. (4.30), that so are the $\left[\bar{F}^{n+1}{ }_{M_{J}+k M_{L_{\lambda}}}^{L}+j\right]_{J^{\prime} L^{\prime} d^{\prime}}$, the corresponding quantum numbers being $J, M_{J}+k, L$, and $M_{L_{\lambda}}+j$. As a result of this important property, we can omit the bar on these $F$, add a parity index $\Pi^{\prime}=\Pi+1 \bmod 2$ associated with $n+1$ (since $\Pi$ is associated with $n$ ), and write them simply as $\left[F^{\Pi^{\prime} n+1} \underset{M_{J}+k M_{L_{\lambda}+j}^{J}}{L}\right]_{J^{\prime} L^{\prime} d^{\prime}}$, where the indices $J^{\prime}, L^{\prime}, d^{\prime}$ indicate that they are expressed, through Eq. (4.30), in terms of the $\mathcal{F}$ functions defined by Eqs. (4.26) and (4.27), which contain those indices. A superscript $D(n+1, J, L)$ will be attached to these functions, as well as a modified $d$ subscript, and the subscripts $J^{\prime}, L^{\prime}, d^{\prime}$ will be dropped, after they are required to be linearly independent, as described in Sec. V B. In order to calculate the $\nabla^{2}\left\{\rho^{n+1}\left[\mathcal{F}^{n+1}{ }_{M_{J}+k}^{J} M_{L_{\lambda}}\right]_{J^{\prime} L^{\prime} d^{\prime}}\right\}$ that appears in the right-hand side of Eq. (4.30), we use for $\nabla^{2}$ the expression,

$$
\nabla^{2}=-\frac{2 \mu}{\hbar^{2}} \hat{T}_{\rho}(\rho)-\frac{\hat{\wedge}^{2}}{\hbar^{2} \rho^{2}}
$$

easily derived from Eq. (2.13). Since $\left[\mathcal{F}^{n+1}{ }_{M_{J}+k}^{J} \stackrel{L}{M_{L_{\lambda}}}\right]_{J^{\prime} L^{\prime} d^{\prime}}$ is independent of $\rho$, replacement of Eq. (4.31) into Eq. (4.30) and dropping the bar in its left-hand side as just justified gives monic polynomials of degree $n+1$ in the $t_{\lambda j}{ }^{k}$, we conclude 


$$
\begin{aligned}
{\left[F^{\Pi^{\prime} n+1} \underset{M_{J}+k M_{L_{\lambda}}+j}{L}\right]_{J^{\prime} L^{\prime} d^{\prime}}(\theta, \phi) } \\
=\frac{1}{2(2 n+7)}\left[\frac{\hat{\wedge}^{2}}{\hbar^{2}}-(n-1)(n+6)\right] \\
\quad \times\left[\mathcal{F}^{n+1}{ }_{M_{J}+k M_{L_{\lambda}}}^{L}\right]_{J^{\prime} L^{\prime} d^{\prime}} .
\end{aligned}
$$

It is useful to define the functions $\left[G^{n+1}\right]_{J^{\prime} L^{\prime} d^{\prime}}$ as the companions of the $\left[F^{\Pi^{\prime} n+1}\right]_{J^{\prime} L^{\prime} d^{\prime}}$ in the relation

$$
\begin{aligned}
& {\left[F^{\Pi^{\prime} n+1} \begin{array}{l}
J \\
M_{J}+k M_{L_{\lambda}}+j_{J^{\prime} L^{\prime} d^{\prime}}
\end{array}\right.} \\
& \left.=(-1)^{(n+1) \chi} \sum_{\Omega_{J_{\lambda}}=-J} \sum_{\Omega_{L_{\lambda}}=-L}^{L} D_{M_{\lambda}}^{J}\right) \\
& \quad \times D_{\Omega_{L_{\lambda}} M_{L_{\lambda}}+k \Omega_{J_{\lambda}}}^{L}\left(\mathbf{\delta}_{\lambda}\right)\left[G^{n+1}{ }_{\Omega_{J_{\lambda}} \Omega_{L_{\lambda}}^{L}}^{L}\right]_{J^{\prime} L^{\prime} d^{\prime}}(\theta, \phi) .
\end{aligned}
$$

It is now desirable to relate the $\left[G^{n+1}\right]_{J^{\prime} L^{\prime} d^{\prime}}$ above to the $\left[\mathcal{G}^{n+1}\right]_{J^{\prime} L^{\prime} d^{\prime}}$ of Eq. (4.26), since the latter have already, by Eq. (4.27), been expressed in terms of the $G^{n}$. This will, as a final result, generate the desired recursion relation between $\left[G^{n+1}\right]_{J^{\prime} L^{\prime} d^{\prime}}$ and $G^{n}$. To relate the $\left[G^{n+1}\right]_{J^{\prime} L^{\prime} d^{\prime}}$ to the $\left[\mathcal{G}^{n+1}\right]_{J^{\prime} L^{\prime} d^{\prime}}$ it suffices to replace, in Eq. (4.30), its lefthand side by Eq. (4.33) and, in its right-hand side, use Eq. (4.26). We then express $\hat{\wedge}^{2}$ in terms of the differential operators $\hat{K}^{2}, \hat{B}, \hat{J}^{2}, \hat{J}_{3}^{I_{\lambda}}, \hat{J}_{ \pm}^{I_{\lambda}}, \hat{L}^{2}, \hat{L}_{\lambda_{3}}$, and $\hat{L}_{\lambda \pm}$, as was done in Appendix A, after Eq. (A12), and use Eqs. (A13)-(A18) together with the orthogonality of the Wigner rotation functions to obtain the expression,

$$
\begin{aligned}
2(2 n+7)\left[G^{n+1}{ }_{\Omega_{J_{\lambda}}} \Omega_{L_{\lambda}}\right. & ]_{J^{\prime} L^{\prime} d^{\prime}}(\theta, \phi) \\
= & {\left[\frac{1}{\hbar^{2}} \hat{K}^{2}(\theta, \phi)+\frac{1}{\hbar^{2}} \hat{B}(\theta, \phi)-(n-1)(n+6)\right] } \\
& \times\left[\mathcal{G}^{n+1}{ }_{\Omega_{J_{\lambda}} \Omega_{L_{\lambda}}^{L}}\right]_{J^{\prime} L^{\prime} d^{\prime}}(\theta, \phi) \\
& +\frac{1}{\hbar^{2}} \sum_{u, v=-2}^{2} V_{\Omega_{J_{\lambda}}^{J L}+u, \Omega_{L_{\lambda}}+v}(\theta, \phi) \\
& \times\left[\mathcal{G}^{n+1}{ }_{\Omega_{J_{\lambda}}+u \Omega_{L_{\lambda}}+v}^{L}\right]_{J^{\prime} L^{\prime} d}(\theta, \phi),
\end{aligned}
$$

where the $V_{\Omega_{J_{\lambda}}+u, \Omega_{L_{\lambda}}+v}^{J L}$ are those defined in Eqs. (A23)(A34). Equation (4.34) together with Eq. (4.27) relates the $\left[G^{n+1}{ }_{\Omega_{J_{\lambda}}}^{J} \Omega_{L_{\lambda}}^{L}\right]_{J^{\prime} L^{\prime} d}(\theta, \phi)$ to the $G_{\Omega_{J_{\lambda}}-p \Omega_{L_{\lambda}}-q d}^{D}(\theta, \phi)$ and therefore permits us to get from a complete set of $G^{n}$ hyperspherical harmonics for a fixed $n$ and all possible values of the remaining six indices, a similarly complete set of $G^{n+1}$ hyperspherical harmonics.

To initiate the iteration procedure, it suffices to have the $n=J=\Omega_{J}=L=\Omega_{L}=0 \quad G$ functions. There is only one linearly-independent solution of Eq. (A21) for this case and it is a constant, which can be set to unity. As a result, $D$ $=1$ and $d=1$ and we can write

$$
G^{0} \begin{array}{llll}
0 & 0 & 1 & 1
\end{array}=1 .
$$

Alternatively, we may start the iterative procedure from the

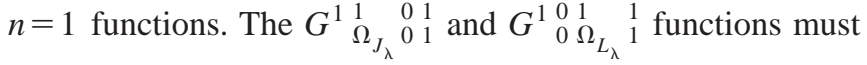

vanish because of Eqs. (3.25) and (3.26). The function $G_{001}^{1001}$ must also vanish. Indeed, for $n=1$ and $J=\Omega_{J}=L=\Omega_{L}=0$, Eq. (A21) becomes

$$
\left[\hat{K}^{2}(\theta, \phi)+\hat{B}(\theta, \phi)\right] G^{1} \begin{array}{ccc}
0 & 0 & 1 \\
0 & 0 & 1
\end{array}(\theta, \phi)=8 \hbar^{2} G^{1} \begin{array}{llll}
0 & 0 & 1 \\
0 & 0 & 1
\end{array}(\theta, \phi)
$$

which means that $\left.G^{10001} \quad 00, \phi\right)$ is an eigenfunction of $\left[\hat{K}^{2}\right.$ $+\hat{B}$ ] with eigenvalue $8 \hbar^{2}$. Obviously, $G^{1} \begin{array}{llll}0 & 0 & 1 \\ 0 & 0 & 1\end{array}=0$ is a solution of Eq. (4.36). This equation has furthermore no additional solutions which are regular at the poles of that operator. ${ }^{19}$ The functions for $n=J=L=1$ can be seen, from Eqs. (4.14) and (3.18), to be nondegenerate [i.e., $D(1,1,1)$ $=1]$ and given by

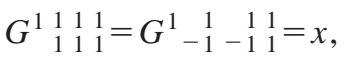

$$
\begin{aligned}
& G^{1}-1111=G^{1} \begin{array}{rrr}
1 & 1 & 1
\end{array}=y, \\
& G^{1} \begin{array}{llll}
1 & 1 & 1 & 1 \\
0 & 0 & 1
\end{array}=z .
\end{aligned}
$$

In this way, the $G_{\Omega_{J_{\lambda}} \Omega_{L_{\lambda}}^{J}}^{L}{ }_{d}^{D}(\theta, \phi)$ for all possible $J, \Omega_{J_{\lambda}}, L$, $\Omega_{L_{\lambda}}, D$ and $d$ are known, and this start-up procedure gives the same results as the one defined by Eq. (4.35), since the first iteration of the latter results in Eqs. (4.37)-(4.39) and in

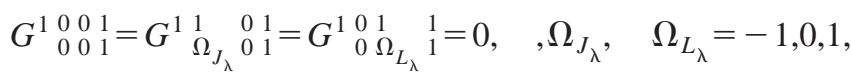

as required.

The indices $J^{\prime}, L^{\prime}$ appearing in the $\left[G^{n+1}{ }_{\Omega_{J_{\lambda}}}^{J} \Omega_{L_{\lambda}}^{L}\right]_{J^{\prime} L^{\prime} d^{\prime}}(\theta, \phi)$ can for a given $J$ and $L$ assume the sets of values given by Eq. (4.28) (which for $J>0$ and $L>0$ are nine sets). This generates a branching tree making the number of $G^{n+1}$ functions grow very rapidly with $n$. However, not all of the resulting $\left[G^{n+1} J_{J_{\lambda}} \Omega_{L_{\lambda}}\right]_{J^{\prime} L^{\prime} d^{\prime}}$ sets (each set scanned by varying the values of $\Omega_{J_{\lambda}}$ and $\Omega_{L_{\lambda}}$ ) are linearly-independent, as described in Sec. V B. Before proceeding to the calculation of the $G^{n+2}$, the $G^{n+1}$ must be culled in order to retain only linearly-independent sets. This is accomplished with the help of a separate routine, also written in Mathematica. It should also be noticed that since the $G^{n}$ functions generate the $G^{n+1}$ ones, they are associated with $F^{n}$ and $F^{n+1}$ functions which have opposite parity. In solving scattering problems, however, matrix elements of the system's potential energy function in the $F$ basis set will appear. For two such $F$ functions of different parity, those matrix elements vanish, as discussed after Eq. (3.18). As a result, the $n$ even and $n$ odd sets of $F$ functions do not mix in the scattering equations, even though they are generated by the recursion relation in the mixed manner just described.

The recursion relation of Eqs. (4.27), (4.34), and (4.35) have been implemented using the Mathematica computer algebra program. ${ }^{28}$ In doing so it was necessary, in Eq. (4.34), to have this program calculate analytically the term $\left(\hat{K}^{2}\right.$ $+\hat{B})\left[\mathcal{G}^{n+1} \underset{\Omega_{J_{\lambda}}}{J} \Omega_{L_{\lambda}}^{L}\right]_{J^{\prime} L^{\prime} d^{\prime}}$ where the differential operators $\hat{K}^{2}$ and $\hat{B}$ are given by Eqs. (2.18)-(2.21). This calculation involves differentiations of trigonometric functions of $\theta$ and $\phi$ with respect to these two angles. Although Mathematica can perform such differentiations, it is not very nimble in simpli- 
fying the results by grouping appropriately terms interrelated by trigonometric identities. To overcome this difficulty we used the intermediate variables $x, y$, and $z$ defined by Eqs. (4.15) -(4.17) together with Eq. (2.6). As shown in Appendix $\mathrm{E}$, the $G$ functions are homogeneous polynomials of degree $n$ in those variables. We expressed $\hat{K}^{2}+\hat{B}$ [see Eqs. (E5) and (E6)] in terms of $\partial / \partial x, \partial / \partial y$, and $\partial / \partial z$ and programmed Mathematica to apply these operators to the appropriate $\mathcal{G}^{n}$ and to simplify the resulting polynomials (treating $x, y$, and $z$ as independent variables), which it does very efficiently. The final answers can, when desired, be reconverted to functions of $\theta$ and $\phi$. This approach resulted in a very efficient procedure for generating the $G$ functions, as discussed in Sec. VI A.

\section{NORMALIZATION AND DEGENERACY OF THE PRINCIPAL-AXES-OF-INERTIA HYPERSPHERICAL HARMONICS $F$ AND $G$}

In this section we describe how complete sets of normalized linearly-independent hyperspherical harmonics $F$ and $G$ functions are obtained.

\section{A. Prenormalization of the $G$ hyperspherical harmonics}

As shown in Appendix E, the $G$ functions are homogeneous polynomials in the variables $x, y$, and $z$ defined in Eqs. (4.15)-(4.17). The iterative step described by Eq. (4.34), as implemented by a Mathematica program, generates the functions

$$
\begin{aligned}
& {\left[G^{n+1} \Omega_{\Omega_{\lambda}}^{J} \Omega_{L_{\lambda}}^{L}\right.} \\
& \quad]_{J^{\prime} L^{\prime} d^{\prime}}(\theta, \phi) \\
& \quad=A_{J^{\prime} L^{\prime} d^{\prime}}^{n+1} \Omega_{J_{\lambda}}^{J} \Omega_{L_{\lambda}}^{L} \sum_{\substack{i, j, k=1 \\
i+j+k=n+1}}^{n+1}\left(a_{J^{\prime} L^{\prime} d^{\prime}}^{n+1} \Omega_{J_{\lambda}} \Omega_{L_{\lambda}}\right)_{i j k} x^{i} y^{j} z^{k},
\end{aligned}
$$

where the $a$ coefficients are all integers and $A$ is the product of a rational number by the square root of another rational number. Those two rational numbers are generated exactly by that program. These characteristics of $a$ and $A$ stem from the properties of the Clesch-Gordan coefficients that appear in Eq. (4.27). On purpose, the superscript $D$ and subscript $d$ do not yet appear in Eq. (5.1), since those indices refer to the degeneracy and linear independence properties of the $F^{n+1}$ functions which will only be imposed in Sec. VB. As the $G$ functions are always used in connection with the associated $F$ functions of Eq. (3.16), any common multiplicative constant for a set of $G$ functions for fixed $n, J, L$, and spanned by $\Omega_{L_{\lambda}}$ and $\Omega_{L_{\lambda}}$, can be factored out of the sum in that equation and incorporated into the associated normalization constant $N$. As a result, in a prenormalization of the $G$ functions we replace Eq. (5.1) by

$$
\begin{aligned}
& {\left[\begin{array}{lll}
G^{n+1} & J & L \\
\Omega_{J_{\lambda}} \Omega_{L_{\lambda}}
\end{array}\right]_{J^{\prime} L^{\prime} d^{\prime}}(\theta, \phi)} \\
& \quad=B_{J^{\prime} L^{\prime} d^{\prime}}^{n+1} \Omega_{J_{\lambda}}^{J} \Omega_{L_{\lambda}}^{L} \sum_{\substack{i, j, k=1 \\
i+j+k=n+1}}^{n+1}\left(a_{J^{\prime} L^{\prime} d^{\prime}}^{n+1} \Omega_{J_{\lambda}} \Omega_{L_{\lambda}}\right)_{i j k} x^{i} y^{j} z^{k},
\end{aligned}
$$

where

$$
\begin{aligned}
& B_{J^{\prime} L^{\prime} d^{\prime}}^{n+1} \Omega_{J_{\lambda}} \Omega_{L_{\lambda}}^{L} \\
& \quad= \begin{cases}A_{J^{\prime} L^{\prime} d^{\prime}}^{n+1} \Omega_{J_{\lambda}} \Omega_{L_{\lambda}} / A_{J^{\prime} L^{\prime} d^{\prime} J L} J_{L}, & \text { for } J+L \text { even } \\
A_{J^{\prime} L^{\prime} d^{\prime}}^{n+1} \Omega_{J_{\lambda}} \Omega_{L_{\lambda}} / \Omega_{J^{\prime} L^{\prime} d^{\prime} J L-1}^{n+1}, & \text { for } J+L \text { odd. }\end{cases}
\end{aligned}
$$

The $B$ coefficients are smaller than the corresponding $A$ ones and make the elimination of the linearly dependent sets of $G^{n+1}$ functions, performed exactly by a Mathematica program and described in Sec. V B, more efficient. As $n$ becomes large (of the order 30), efficiency becomes important and justifies this prenormalization.

\section{B. Degeneracy of the $F$ and $G$ hyperspherical harmonics}

As discussed after Eq. (3.15), it is expected that for a fixed set of quantum numbers $\Pi, n, J, M_{J}, L$, and $M_{L_{\lambda}}$ there should be more than one $F$ function. We label the latter with the extra degeneracy subscript $d$, as $F^{\Pi n J}{ }_{M_{J} M_{L_{\lambda}}}^{L} d$ where $D$ indicates the total number of these functions, and therefore represents their degeneracy. Let us show that this degeneracy is independent of $M_{J}$ and $M_{L}$. Indeed, by definition, for two functions $F^{\Pi n J}{ }_{M_{J}{ }_{L_{\lambda_{1}}}^{L}}^{L}$ and $F^{\Pi n J}{ }_{M_{J}}{ }_{M_{L_{\lambda_{2}}}}^{L}$ (having the same set of quantum number $\Pi, n, J, L, M_{J}$, and $M_{L_{\lambda}}$ ) to be degenerate, they must be linearly independent. Because of the orthogonality properties of the $D_{M_{J} \Omega_{J_{\lambda}}}^{J}\left(\mathbf{a}_{\lambda}\right)$ and $D_{\Omega_{J_{\lambda}} M_{L_{\lambda}}}\left(\boldsymbol{\delta}_{\lambda}\right)$ functions that appear in Eq. (3.16), a necessary and sufficient condition for that linear independence is that the corresponding sets of $G$ functions $\left\{G^{n J}{ }_{\Omega_{J_{\lambda}}} \Omega_{L_{\lambda_{1}}}\right\}$ and $\left\{G^{n}{ }_{\Omega_{J_{\lambda}}} \Omega_{L_{\lambda_{2}}}\right\}$ (each set spanned by the values of $\Omega_{J_{\lambda}}$ and $\Omega_{L_{\lambda}}$ ), be linearly independent. By this we mean that it is necessary and sufficient that at least one $\bar{\Omega}_{J_{\lambda}}$ and $\bar{\Omega}_{L_{\lambda}}$ exist for a given $n, J$, and $L$ for which the functions $G^{n}{ }^{J} \bar{\Omega}_{J_{\lambda}} \bar{\Omega}_{L_{\lambda_{1}}}$ and $G^{n} \bar{\Omega}_{J_{\lambda}}^{J} \bar{\Omega}_{L_{\lambda_{2}}}$ are linearly independent. Since the $G$ functions, as discussed after Eq. (3.16), are independent of $M_{J}$ and $M_{L_{\lambda}}$, this linearindependence condition does not depend on these quantum numbers. Therefore, neither does $D$, Q.E.D. $D$ can however depend on $n, J$, and $L$, and as shown in the rest of this section, does indeed depend on these three quantum numbers. It should be stressed that $D$, in addition to representing the degeneracy of the $F^{\Pi n J}{ }_{M_{J} M_{L_{\lambda}} d}{ }^{D}$ functions, also represents

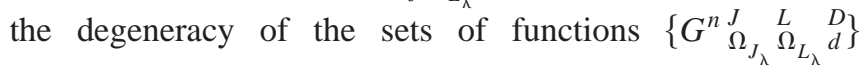
spanned by $\Omega_{J_{\lambda}}$ and $\Omega_{L_{\lambda}}$. 
Let us now consider the functions $\left[G^{n+1}{ }_{\Omega_{J_{\lambda}}}^{J} \Omega_{L_{\lambda}}^{L}\right]_{J^{\prime} L^{\prime} d^{\prime}}(\theta, \phi)$ generated by Eq. (4.34) [and expressed in the form of Eq. (5.2)] with $J^{\prime}, L^{\prime}$ assuming all sets of values permitted by Eq. (4.28) for a given $J$ and $L$. Since these functions do not yet satisfy the linearindependence condition described in the preceding paragraph, the indices $D$ and $d$ on $G^{n+1}{ }_{\Omega_{J_{\lambda}}}^{J} \Omega_{L_{\lambda}}^{L} \quad{ }^{D}$ have been temporarily omitted, as discussed after Eq. (5.1). The index $d$ has instead been replaced, in Eq. (5.2), by the set of subscripts $J^{\prime}, L^{\prime}$, and $d^{\prime}$, where $d^{\prime}$ refers to the index in the function $G^{n}{ }_{\Omega_{J_{\lambda}^{\prime}}-p} \Omega_{L_{\lambda}^{\prime}-q d^{\prime}} D^{\prime}(\theta, \phi)$ that appears in the righthand side of Eq. (4.27); this $d^{\prime}$ differs from the one that will eventually be attached to $G^{n+1} \underset{\Omega_{J_{\lambda}}^{J}}{\stackrel{L}{L} \Omega_{L_{\lambda}}} \underset{d}{D}(\theta, \phi)$. Let $\mathbf{b}_{J^{\prime} L^{\prime} d^{\prime}}^{n+1 J L}$ be the column vector whose elements

$$
\left(b_{J^{\prime} L^{\prime} d^{\prime}}^{n+1} \stackrel{J}{\Omega_{J_{\lambda}}} \stackrel{L}{\Omega_{L_{\lambda}}}\right)_{i j k}=B_{J^{\prime} L^{\prime} d^{\prime}}^{n+1} \stackrel{J}{\Omega_{J_{\lambda}}} \stackrel{L}{\Omega_{L_{\lambda}}}\left(a_{J^{\prime} L^{\prime} d^{\prime}}^{n+1} \Omega_{J_{\lambda}}^{J} \Omega_{L_{\lambda}}^{L}\right)_{i j k}
$$

are spanned by the set of five indices $\Omega_{J_{\lambda}}, \Omega_{L_{\lambda}}, i, j, k$ such that

$$
i+j+k=n+1
$$

with $i, j, k$ being non-negative integers. The number of solutions of Eq. (5.5) is

$$
M_{n+1}=(n+2)(n+3) / 2 \text {. }
$$

As can be seen, $M_{n+1}$ increases quadratically with $n$, reaching the value 528 for $n=30$. It should be realized, however, that many of the corresponding $\left(b_{J^{\prime} L^{\prime} d^{\prime}}^{n+1} \Omega_{J_{\lambda}}^{J} \Omega_{L_{\lambda}}^{L}\right)_{i j k}$ equal zero, as can be seen in the particular but representative case depicted in Table IV, for which $n+1=10, J=8, \Omega_{J_{\lambda}}=1, L$ $=4$, and $\Omega_{L_{\lambda}}=-1$. For this case $M_{10}$ equals 66 , but only 19 of the corresponding $\left(b^{108} \begin{array}{l}4 \\ 1-1\end{array}\right)_{i j k}$ do not vanish. Due to the requirement that $\Omega_{J_{\lambda}}+\Omega_{L_{\lambda}}$ be even, the number of permitted $\Omega_{J_{\lambda}}, \Omega_{L_{\lambda}}$ pairs is given approximately by $(2 J+1)(2 L$ $+1) / 2$. Let $\mathcal{N}^{n+1 J L}$ be the length (i.e., the number of nonzero elements) of the $\mathbf{b}_{J^{\prime} L^{\prime} d^{\prime}}^{n+1 J L}$ vector. In view of Eq. (4.27) and Eq. (4.28), that length does not depend on $J^{\prime}, L^{\prime}$ or $d^{\prime}$. Exceptionally, certain $\left(b_{J^{\prime} L^{\prime} d^{\prime}}^{n+1} \Omega_{J_{\lambda}}^{J} \Omega_{L_{\lambda}}^{L}\right)_{i j k}$ elements may vanish accidentally because of a cancellation of terms in their evaluation, in which case we allow those accidental zeros to be counted as elements of the corresponding $\mathbf{b}_{J^{\prime} L^{\prime} d^{\prime}}^{n+1 J L}$ vector, in order to maintain this property of $\mathcal{N}^{n+1 J L}$. From the considerations above, we conclude that $\mathcal{N}^{n+1 J L}$ is in the range,

$$
M_{n+1}<\mathcal{N}^{n+1 J L} \lesssim(2 J+1)(2 L+1) / 2 .
$$

For example, for $n+1=4$ and $J=L=2$ the value of $\mathcal{N}^{4} 22$ is 42 , whereas $M_{n+1}$ is 15 and $(2 J+1)(2 L+1) / 2$ is 187.5 .

We now consider the set of all vectors $\mathbf{b}_{J^{\prime} L^{\prime} d^{\prime}}^{n+1 J L}$, of equal length $\mathcal{N}^{n+1 J L}$, obtained by allowing $J^{\prime}, L^{\prime}$, and $d^{\prime}$ to assume all of their permitted values. For each such vector we calculate the sum of the absolute values of its elements, and construct the matrix $\mathbf{b}^{n+1 J L}$ whose columns are those vectors placed in increasing order of that sum. This ordering is im- portant for optimizing the efficiency of the procedure that generates the linearly-independent hyperspherical harmonics. The number of columns of this matrix is

$$
\mathcal{M}^{n+1 J L}=\sum_{J^{\prime}=|J-1|}^{J+1} \sum_{L^{\prime}=|L-1|}^{L+1} D^{\prime}\left(n, J^{\prime}, L^{\prime}\right),
$$

where $D^{\prime}\left(n, J^{\prime}, L^{\prime}\right)$ is the degenercy of the $F_{M_{J}^{\prime} M_{L_{\lambda}^{\prime}} d^{\prime}}^{J^{\prime}}$ functions. The dimensions of $\mathbf{b}^{n+1 J L}$ are, therefore, $\mathcal{N}^{n+1 J L} \times \mathcal{M}^{n+1 J L}$. The next step is to contract this matrix to one whose columns are linearly independent. We adopted the following contraction procedure:

(1) Consider the matrix $\mathbf{b}_{1}$ formed by the first two columns of $\mathbf{b}$ and determine its rank $\mathcal{R}_{1}$. If $\mathcal{R}_{1}=1$, replace the second column of $\mathbf{b}_{1}$ by the third column of $\mathbf{b}$. If $\mathcal{R}_{1}$ $=2$, augment $\mathbf{b}_{1}$ by the third column of $\mathbf{b}$. Call the resulting 2 or 3 column matrix $\mathbf{b}_{2}$. Its rank $\mathcal{R}_{2}$ is equal to its number of columns.

(2) Augment $\mathbf{b}_{2}$ by the next column of $\mathbf{b}$, and procede as in step 1 to generate a matrix $\mathbf{b}_{3}$ having 4 or 5 columns depending on whether $\mathcal{R}_{3}$ is 4 or 5 .

(3) Continue the augmentation procedure, one column at a time, until the columns of $\mathbf{b}$ are exhausted.

The resulting final matrix $\mathbf{b}_{D}$ will have rank $D$ and be formed by $D$ linearly-independent columns $\mathbf{b}_{d} \quad(d$ $=1,2, \ldots, D)$. The number $D$ will depend on $n+1, J$ and $L$ only. The rank determination of the matrices $\mathbf{b}_{i}$ is done exactly, with an available Mathematica program. The elements of $\mathbf{b}_{D}$ are now designated by the symbol $\left(b^{n+1} \begin{array}{lll}J & L & D \\ \Omega_{J_{\lambda}} & \Omega_{L_{\lambda}} & d\end{array}\right)_{i j k}$. Inserting them into Eq. (5.2) (with obvious changes in notation) and using Eq. (5.4) we get

$$
G^{n+1} \underset{\Omega_{J_{\lambda}}}{\stackrel{L}{L} \Omega_{L_{\lambda}}} \underset{d}{D}(\theta, \phi)=\sum_{\substack{i, j, k=1 \\ i+j+k=n+1}}^{n+1}\left(b^{n+1} \underset{\Omega_{J_{\lambda}}}{\stackrel{L}{L} \Omega_{L_{\lambda}}} \stackrel{D}{d}\right)_{i j k} x^{i} y^{j} z^{k},
$$

where $D=D(n+1, J, L)$. This constitutes a complete ensemble of $D$ linearly-independent sets of $G$ functions for $n$ +1 (each set spanned by $\Omega_{J_{\lambda}}$ and $\Omega_{L_{\lambda}}$ ), generated starting with a knowledge of a complete ensemble of $G$ functions for $n$. When used in Eq. (3.16) or Eq. (3.18) they result in a complete set of linearly-independent $F$ functions for $n+1$. It should be noticed that, as a consequence of the ordering of the $\mathbf{b}$ vectors described in the paragraph following Eq. (5.7), the $\mathbf{b}_{D}$ matrix will have elements that are much smaller than if the ordering were random. This property significantly speeds up the rank-determination code and generates $G$ functions whose coefficients are smaller and simpler than they would be otherwise.

A check of the correctness of the degeneracy number $D(n, J, L)$ obtained by the procedure just described is provided by the $J=L=0$ case. For that case, Littlejohn et al., ${ }^{19}$ using group theoretic methods, derived the expression

$$
\begin{aligned}
D(n, 0,0)= & 3 / 8+\{[(\nu-1) \bmod 3]-1\} / 3 \\
& +(-1)^{\nu} / 4+(4 \nu+1) / 24,
\end{aligned}
$$


TABLE I. The number of linearly independent hyperspherical harmonics in row-orthonomal hyperspherical coordinates for $n=15$. ${ }^{\text {a }}$

\begin{tabular}{cccccccccccccccccc}
\hline \hline$J$ & $L$ & $D$ & $J$ & $L$ & $D$ & $J$ & $L$ & $D$ & $J$ & $L$ & $D$ & $J$ & $L$ & $D$ & $J$ & $L$ & $D$ \\
\hline 0 & 0 & 2 & 6 & 1 & 7 & 8 & 7 & 15 & 10 & 9 & 8 & 12 & 8 & 4 & 14 & 4 & 1 \\
1 & 1 & 4 & 6 & 2 & 11 & 8 & 8 & 14 & 10 & 10 & 7 & 12 & 9 & 4 & 14 & 5 & 1 \\
2 & 0 & 2 & 6 & 3 & 15 & 9 & 0 & 1 & 11 & 1 & 4 & 12 & 10 & 4 & 14 & 6 & 1 \\
2 & 1 & 4 & 6 & 4 & 18 & 9 & 1 & 6 & 11 & 2 & 4 & 12 & 11 & 3 & 14 & 7 & 1 \\
2 & 2 & 8 & 6 & 5 & 19 & 9 & 2 & 7 & 11 & 3 & 7 & 12 & 12 & 3 & 14 & 8 & 1 \\
3 & 0 & 1 & 6 & 6 & 20 & 9 & 3 & 12 & 11 & 4 & 6 & 13 & 1 & 2 & 14 & 9 & 1 \\
3 & 1 & 7 & 7 & 0 & 1 & 9 & 4 & 12 & 11 & 5 & 8 & 13 & 2 & 2 & 14 & 10 & 1 \\
3 & 2 & 8 & 7 & 1 & 8 & 9 & 5 & 14 & 11 & 6 & 6 & 13 & 3 & 3 & 14 & 11 & 1 \\
3 & 3 & 14 & 7 & 2 & 10 & 9 & 6 & 13 & 11 & 7 & 8 & 13 & 4 & 2 & 14 & 12 & 1 \\
4 & 0 & 3 & 7 & 3 & 16 & 9 & 7 & 14 & 11 & 8 & 6 & 13 & 5 & 3 & 14 & 13 & 1 \\
4 & 1 & 6 & 7 & 4 & 16 & 9 & 8 & 11 & 11 & 9 & 7 & 13 & 6 & 2 & 14 & 14 & 1 \\
4 & 2 & 11 & 7 & 5 & 20 & 9 & 9 & 11 & 11 & 10 & 5 & 13 & 7 & 3 & 15 & 1 & 1 \\
4 & 3 & 13 & 7 & 6 & 18 & 10 & 0 & 1 & 11 & 11 & 6 & 13 & 8 & 2 & 15 & 3 & 1 \\
4 & 4 & 17 & 7 & 7 & 19 & 10 & 1 & 4 & 12 & 0 & 1 & 13 & 9 & 3 & 15 & 5 & 1 \\
5 & 0 & 1 & 8 & 0 & 2 & 10 & 2 & 6 & 12 & 1 & 2 & 13 & 10 & 2 & 15 & 7 & 1 \\
5 & 1 & 8 & 8 & 1 & 6 & 10 & 3 & 8 & 12 & 2 & 3 & 13 & 11 & 3 & 15 & 9 & 1 \\
5 & 2 & 11 & 8 & 2 & 10 & 10 & 4 & 9 & 12 & 3 & 4 & 13 & 12 & 2 & 15 & 11 & 1 \\
5 & 3 & 16 & 8 & 3 & 12 & 10 & 5 & 10 & 12 & 4 & 4 & 13 & 13 & 2 & 15 & 13 & 1 \\
5 & 4 & 17 & 8 & 4 & 15 & 10 & 6 & 10 & 12 & 5 & 4 & 14 & 1 & 1 & 15 & 15 & 1 \\
5 & 5 & 21 & 8 & 5 & 16 & 10 & 7 & 9 & 12 & 6 & 4 & 14 & 2 & 1 & & & \\
6 & 0 & 3 & 8 & 6 & 16 & 10 & 8 & 9 & 12 & 7 & 4 & 14 & 3 & 1 & & \\
\hline \hline
\end{tabular}

${ }^{\mathrm{a}}$ For $L \leqslant J$. For $L>J$, the relation $D(n, L, J)=D(n, J, L)$ is used.

where $\nu=n / 2$ for $n$ even and $\nu=(n-3) / 2$ for $n$ odd. That expression has not yet been generalized to other values of $J$ and $L$. Our results agree with Eq. (5.10) for the range of $n$ $=1-30$ for which we performed calculations. Another and more stringent check was made as follows. Avery ${ }^{29}$ derived a general expression for calculating the total number $N_{n}$ of linearly-independent hyperspherical harmonics $F$ for a given $n$,

$$
N_{n}=\frac{(2 n+7)(n+6) !}{n ! 7 !} .
$$

This number is related to $D(n, J, L)$ by

$$
N_{n}=\sum_{J, L=0}^{n}(2 J+1)(2 L+1) D(n, J, L) .
$$

The largest degeneracy we encountered in our calculations was $D(30,14,14)=127$. We checked the correctness of the $D(n, J, L)$ obtained by our recursion relation procedure against Eqs. (5.11) and (5.12). We calculated the values of $D$ for all $n$ up to 30 and perfect agreement was obtained between those two equations. As an example, a representative set of values of $D(n, J, L)$ (for $n=15$ ) is given in Table I. From this table and Eq. (5.12), as well as from Eq. (5.11), one gets $N_{15}=286824$. Taking into account that $N_{n}$ varies from 1 for $n=0$ to 18643152 for $n=30$, such an agreement is strongly suggestive that the recursion relations used and the codes written to implement them are indeed correct.

Finally, an ultimate check was performed on the correctness of all the $G^{n} \Omega_{J_{\lambda}}^{J} \Omega_{L_{\lambda}} d$ generated by the procedure described. For each set of values of $n, J, L, D$, and $d$, these functions (for all $\Omega_{J_{\lambda}}, \Omega_{L_{\lambda}}$ ) were replaced in both sides of Eq. (A21) and shown to satisfy it with the help of a Mathematica program $^{28}$ written for this purpose. This test was done for all $G$ with $n$ up to 30 , and which, as mentioned in Sec. V A, amounted to about 43.8 million functions. Thus, with the correctness of the degeneracy parameter $D(n, J, L)$ and of the $G$ hyperspherical harmonics verified independently, it is concluded that they are both free of error.

\section{Normalization and orthogonality of the $F$ and $G$ hyperspherical harmonics}

It is desirable to normalize the $F$ functions according to

$$
\begin{aligned}
& \int\left[\left|F^{\Pi n}{ }_{M_{J} M_{L_{\lambda}}}^{L}{ }_{d}^{D}\left(\chi=0, \boldsymbol{\Theta}_{\lambda}\right)\right|^{2}\right. \\
& \left.+\left|F^{\Pi n}{ }_{M_{J} M_{L_{\lambda}}}^{L}{ }_{d} d\left(\chi=1, \boldsymbol{\Theta}_{\lambda}\right)\right|^{2}\right] d \boldsymbol{\Theta}_{\lambda}=1,
\end{aligned}
$$

where

$$
\begin{aligned}
d \Theta_{\lambda}= & \sin b_{\lambda} d a_{\lambda} d b_{\lambda} d_{\lambda} \sin \delta_{\lambda}^{(2)} d \delta_{\lambda}^{(1)} d \delta_{\lambda}^{(2)} d \delta_{\lambda}^{(3)} \\
& \times g(\theta, \phi) \sin \theta d \theta d \phi
\end{aligned}
$$

and $^{17}$

$$
\begin{aligned}
g(\theta, \phi)= & \sin ^{2} \theta \cos 2 \phi\left(\cos ^{2} \theta-\sin ^{2} \theta \sin ^{2} \phi\right) \\
& \times\left(\cos ^{2} \theta-\sin ^{2} \theta \cos ^{2} \phi\right) .
\end{aligned}
$$

The ranges of the $\boldsymbol{\delta}_{\lambda}$ angles in the integral of Eq. (5.13) are

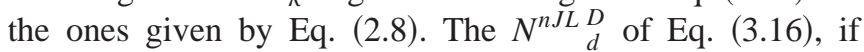
chosen to be real and positive should, as a result, be 


$$
\begin{aligned}
N_{d}^{n J L} \underset{d}{D}= & \left\{\frac{32 \pi^{4}}{(2 J+1)(2 L+1)} \sum_{\Omega_{J_{\lambda}}=-J}^{J} \sum_{\Omega_{L_{\lambda}}=-L}^{L} \int_{0}^{\pi / 4} d \phi\right. \\
& \left.\times \int_{0}^{\theta(\phi)} d \theta\left[G_{\Omega_{J_{\lambda}} \Omega_{L_{\lambda}}^{J}}^{L}{ }_{d}^{D}(\theta, \phi)\right]^{2} g(\theta, \phi) \sin \theta\right\}^{-1 / 2},
\end{aligned}
$$

where

$$
\theta(\phi)=\arcsin \left[1 /\left(1+\cos ^{2} \phi\right)^{1 / 2}\right] \quad 0 \leqslant \theta \lesssim 54.7^{\circ} .
$$

Since the normalization coefficient $N^{n J L}{ }_{d}^{D}$ is independent of $M_{J}$ and $M_{L_{\lambda}}$, we can use it to define the modified function $\bar{G}$ by

$$
\bar{G}^{n}{\underset{\Omega}{J_{\lambda}} \Omega_{L_{\lambda}}^{J}}_{d}^{L} \quad \underset{D}{D}(\theta, \phi)=N^{n J L} \underset{d}{D} G_{\Omega_{J_{\lambda}}^{n}}^{J} \Omega_{L_{\lambda}}^{L} \underset{d}{D}(\theta, \phi) .
$$

The magnitudes of these normalized $\bar{G}$ functions are considerably smaller than those of the corresponding $G$ functions, which is a convenient property. It is important to stress that the functions $F^{\Pi n J}{ }_{M_{J}}^{L} M_{L_{\lambda}} d$, which are orthogonal with respect to $\Pi, n, J, M_{J}, L$, and $M_{L_{\lambda}}$, are not orthogonal with respect to $d$ even after normalized according to Eq. (5.13). If desired, they can be orthogonalized with respect to this quantum number by a Gram-Schmidt or some other orthogonalization procedure. Similarly, the set of functions $G_{\Omega_{J_{\lambda}}}^{J} \Omega_{L_{\lambda}}^{L} d$, for a fixed $n, J, L$, and $D$, are not orthogonal with respect to any of the quantum number $\Omega_{J_{\lambda}}, \Omega_{L_{\lambda}}$, and $d$. Again, they may be orthogonalized if desired.

\section{REPRESENTATIVE RESULTS}

\section{A. General considerations}

We used the procedure described in Sec. V to generate all the hyperspherical harmonic functions $G^{n}{ }_{\Omega_{J_{\lambda}}}^{J} \Omega_{L_{\lambda}}^{L}{ }_{d}^{D}(\theta, \phi)$ for $n$ from 0 to 30 . For each $n$, the number of $G$ functions is approximately one-half the $N_{n}$ of Eq. (5.11). The reason for this decrease is that $N_{n}$ is the number of linearly independent $F^{\Pi n J}{ }_{M_{J} M_{L_{\lambda}}{ }_{d}}^{D}{ }^{D(n, J, L)}$ functions. Whereas $M_{J}$ can assume all values between $-J$ and $J$ and $M_{L_{\lambda}}$ all values between $-L$ and $L$, the values of $\Omega_{J_{\lambda}}$ and $\Omega_{L_{\lambda}}$, although in the same range, are restricted by the additional condition that $\Omega_{J_{\lambda}}$ $+\Omega_{L_{\lambda}}$ be even. As a result, the total number of nonvanishing $G$ functions generated for all those $n$ was about 43.8 million. The five values of $n$ of 26-30 accounted for about 31.6 million of those functions.

The calculation of those 43.8 million functions was performed on a Dell desktop computer operating with a 450 $\mathrm{MHz}$ Pentium II processor, and required about one month of total running time. Given the independence of these functions on the characteristics of the tetraatomic system for which they will be used, they will not have to calculated again, except for obtaining more of them when needed. They are expressed in the form of Eq. (5.9), where the $b$ coefficients have been normalized as described by Eq. (5.4). These coefficients are stored as sets of values for fixed $n, J, \Omega_{J_{\lambda}}$, $L, \Omega_{L_{\lambda}}$, and $d$, the indices $i, j, k$ scanning each set.
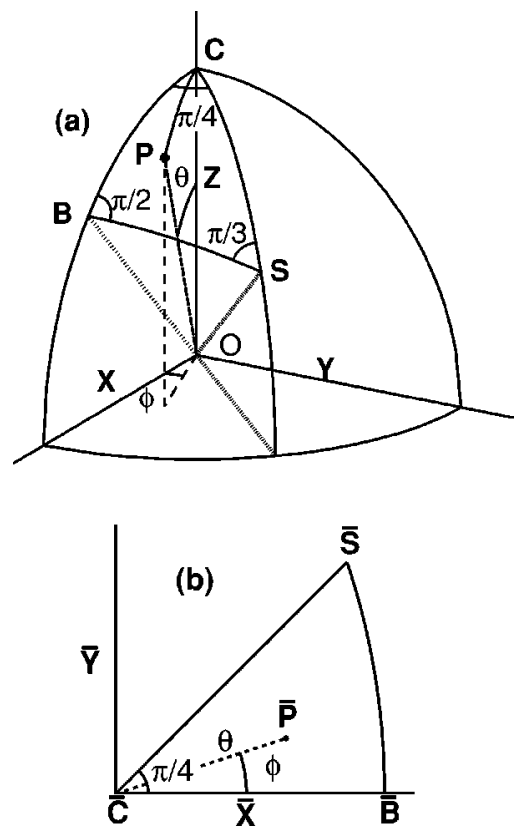

FIG. 1. The unit sphere in $\rho, \theta, \phi$ space. Panel (a) depicts a system of Cartesian axes OXYZ and one-eighth of a unit sphere. A general point $\mathrm{P}$ on that sphere has spherical polar coordinates $\rho=1, \theta, \phi$. The points $\mathrm{C}(1,0,0)$, $\mathrm{B}(1, \pi / 4,0)$ and $\mathrm{S}\left(1, \arcsin (2 / 3)^{1 / 2}, \pi / 4\right)$ are the vertices of a spherical triangle whose sides are the arcs of great circle $\overparen{\mathrm{CB}}, \overparen{\mathrm{BS}}, \overparen{\mathrm{SC}}$ and whose internal angles are $\pi / 4, \pi / 2$, and $\pi / 3$. Panel (b) shows a mapping of that unit sphere onto a plane $\bar{C} \bar{X} \bar{Y}$ defined by Eqs. (6.5) and (6.6) in the text. The figure whose sides are the straight lines $\bar{C} \bar{B}$ and $\bar{C} \bar{S}$ and the curved line $\bar{B} \bar{S}$ is the map of the spherical triangle on that plane. The equation of that curved line is obtained by expressing the $\theta$ in those equations as the function of $\phi$ given by Eq. (6.1)

\section{B. Comparison with previous results}

Limited sets of $G$ functions were obtained by Zickendraht ${ }^{20}$ and by Littlejohn et al. ${ }^{19}$ Zickendraht obtained all $G$ functions for $n=1$ and 2, using slightly different variables. When transformed into our variables, the agreement was perfect. [It should be noticed that there is a typographical error in his Eq. (32), in which, using his notation, ${ }_{2} G_{20}^{20}$ should be labeled ${ }_{2} G_{10}^{10}$.] Littlejohn et al. obtained $G$ functions for $1 \leqslant n \leqslant 7$ but restricted to the $J=L=0$ case. Again, the agreement with the present results was perfect.

\section{The unit $\theta, \phi$ sphere}

In order to examine the behavior of the $\bar{G}$ functions, normalized as described in Sec. VC, in terms of the $\theta, \phi$ angles, it is useful to consider the corresponding unit sphere. A point $\mathrm{P}$ on that sphere has spherical polar coordinates $(1, \theta$, $\phi)$, and is depicted in Fig. 1(a). In view of the ranges of $\theta$ and $\phi$ given by Eq. (2.9), the points on that sphere corresponding to allowed configurations of the system are those internal to the spherical triangle CBS, where $C \equiv(1,0,0), B$ $\equiv(1, \pi / 4,0)$, and $S \equiv\left(1, \arcsin (2 / 3)^{1 / 2}, \pi / 4\right)$. The point C represents a collinear configuration which is also a prolate symmetric top, B a coplanar configuration which is also an oblate symmetric top, and $\mathrm{S}$ a spherical top configuration. The arc $\mathrm{CB}$ represents all coplanar configurations, and all of its points have $\phi=0$. The arc CS represents all prolate symmet- 
TABLE II. Principal-axes-of-inertia hyperspherical harmonics $G$ for $n=4$, $J=2, L=1$, and $D=1$.

\begin{tabular}{|c|c|c|}
\hline$\Omega_{J_{\lambda}}$ & $\Omega_{L_{\lambda}}$ & $G^{4} \Omega_{J_{\lambda}}^{2} \stackrel{1}{\Omega_{L_{\lambda}}}{ }_{1}^{1}=G_{-\Omega_{J_{\lambda}}}^{4}{ }_{-\Omega_{L_{\lambda}}}^{1}{ }^{1}$ \\
\hline 1 & -1 & $-\frac{1}{2 \sqrt{2}} y z\left(3 x^{2}+y^{2}-z^{2}\right)$ \\
\hline 1 & 1 & $\frac{1}{2 \sqrt{2}} x z\left(x^{2}+3 y^{2}-z^{2}\right)$ \\
\hline 2 & 0 & $-x y\left(x^{2}-y^{2}\right)$ \\
\hline \multicolumn{2}{|c|}{$N^{42} \underset{1}{1}$} & 20.69189 \\
\hline
\end{tabular}

ric top configurations, and all of its points have $\phi=\pi / 4$. Finally, the $\widehat{\mathrm{BS}}$ arc represents all oblate symmetric top configurations and its equation is

$$
\theta(\phi)=\arcsin \left[1 /\left(1+\cos ^{2} \phi\right)\right]^{1 / 2} .
$$

All three of those arcs are arcs of great circle; the spherical triangle they form has internal angles of $\pi / 4, \pi / 2$, and $\pi / 3$ as indicated on that figure. ${ }^{19}$ The Cartesian coordinates of the point $\mathrm{P}$ on the unit sphere of Fig. 1(a) are

$$
\begin{aligned}
& X=\sin \theta \cos \phi, \\
& Y=\sin \theta \sin \phi, \\
& Z=\cos \theta .
\end{aligned}
$$

It is convenient to map these points onto a $\bar{C} \bar{X} \bar{Y}$ plane by the equations

$$
\begin{gathered}
\bar{X}=\theta \cos \phi, \\
\bar{Y}=\theta \cos \phi .
\end{gathered}
$$

This is equivalent to replacing $\sin \theta$ by $\theta$ in the first two of Eqs. (6.3). That plane is depicted in Fig. 1(b). The points $C$, $\mathrm{B}, \mathrm{S}$ are maped onto $\overline{\mathrm{C}}, \overline{\mathrm{B}}$, and $\overline{\mathrm{S}}$, respectively. The straight line segment $\overline{\mathrm{C}} \overline{\mathrm{B}}$ represents coplanar configurations, the straight line segment $\bar{C} \bar{S}$ prolate symmetric top configurations, and the curve $\overline{\mathrm{B}} \overline{\mathrm{S}}$ oblate symmetric top configurations. This mapping of the unit sphere onto a plane preserves the lengths of the arcs of circle whose origin is C in Fig. 1(a), i.e., the length of the arc $\widehat{\mathrm{CP}}$ in that figure equals the length of the straight line segment $\overline{\mathrm{C}} \overline{\mathrm{P}}$ in Fig. 1(b), both being equal to $\theta$; it is a very useful mapping and will be adopted in all of the subsequent figures of this paper.

\section{Hyperspherical harmonics for $n=4$}

The $G$ functions for $n=4, J=2$, and $L=1$ are presented in Table II. The value of $D(4,2,1)$ is equal 1 , i.e., these functions are not degenerate. In view of the symmetry relation Eq. (3.26), only three formulas are given. The corresponding normalization coefficient, calculated using Eq. (5.16), is displayed at the bottom of the table. The associated $\bar{G}$ functions, defined by Eq. (5.18), are plotted in Fig. 2 as a function of $\theta$ and $\phi$. The coordinates correspond to Fig. 1(b) and are depicted on the large circle on the bottom plane of each panel, in which the dashed radial lines correspond to
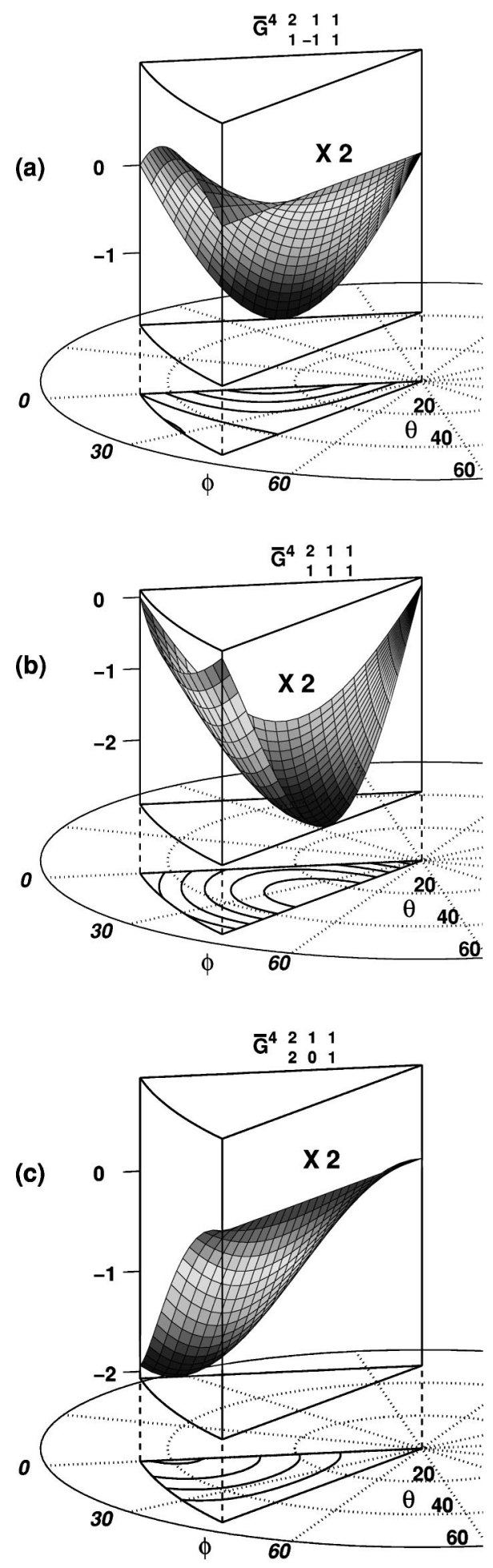

FIG. 2. The hyperspherical harmonics $\bar{G}^{4}{ }_{\Omega_{J_{\lambda}}}^{2} \Omega_{L_{\lambda}}{ }_{1}{ }_{1}^{1}$ as a function of the principal angles of inertia $\theta$ and $\phi$ [displayed in Fig. 1(b)]. The dashed radial lines on the horizontal planes at the bottom of the three panels are constant $\phi$ lines, and the dashed circles are constant $\theta$ lines. The curved solid lines on those planes are contours of constant $\bar{G}$. The multiplicative factor $\times 2$ indicated is the number by which the ordinate was multiplied before being plotted. The normalization factor $\mathcal{N}$ of Eq. (5.16) is 20.6919 for all panels. (a) $\Omega_{J_{\lambda}}=1, \Omega_{L_{\lambda}}=-1$. The values of the scaled $\bar{G}$ (i.e., after the multiplicative factor is applied to $\bar{G}$ ) for the contour lines vary from -1.5 (for the contour closest to the $0^{\circ}$ line) to 0.5 in steps of 0.5 . (b) $\Omega_{J_{\lambda}}=1, \Omega_{L_{\lambda}}=1$. Scaled contour values of -2.5 through 0 in steps of 0.5 . (c) $\Omega_{J_{\lambda}}=2, \Omega_{L_{\lambda}}$ $=0$. Scaled contour values from -2 to -0.5 in steps of 0.5 . 

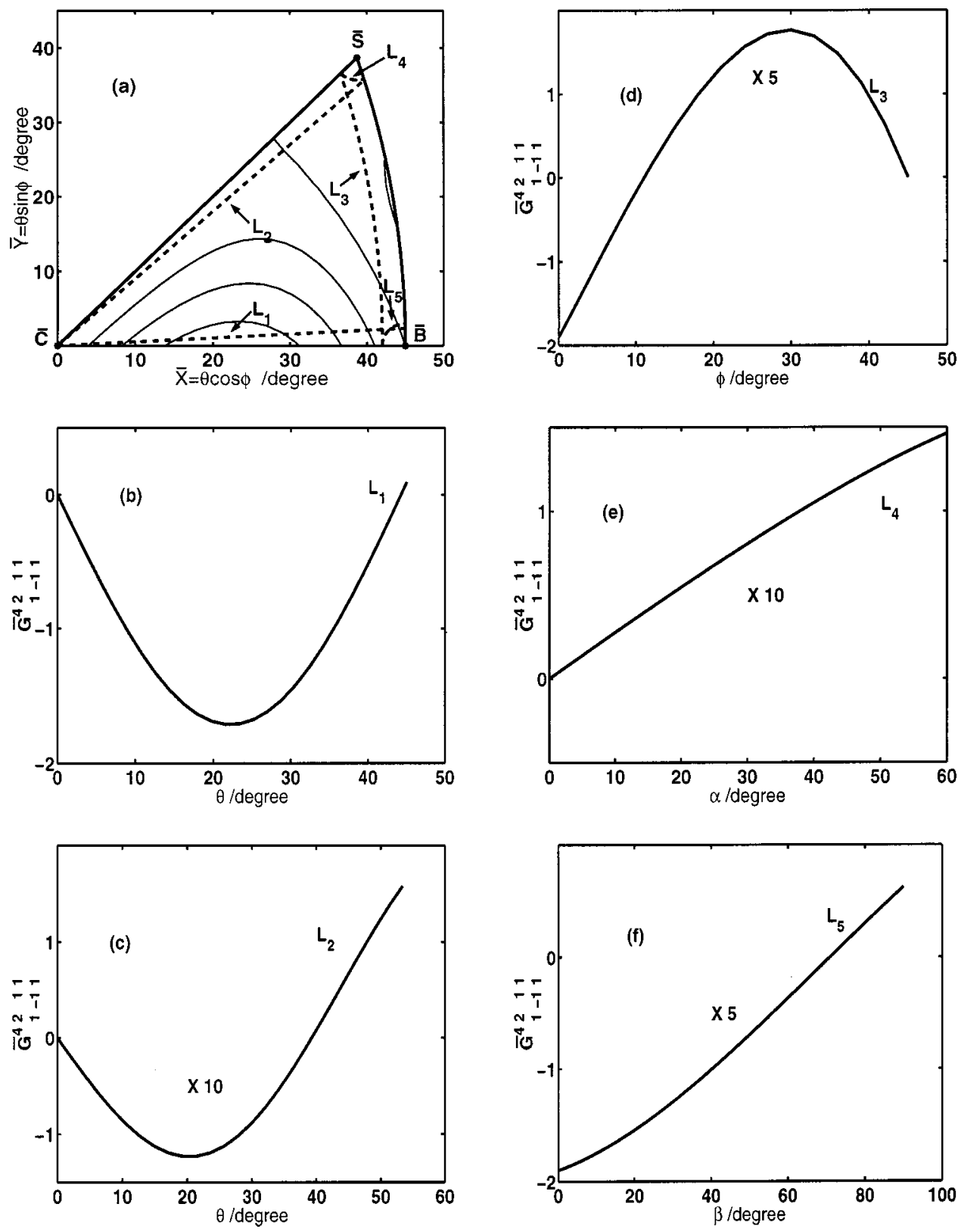

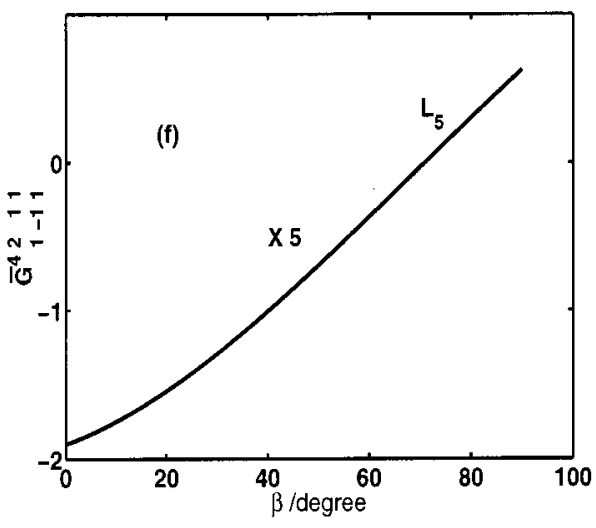

FIG. 3. One-dimensional cuts of $\bar{G}^{4}{ }_{1-1}^{2} 1 \frac{1}{1}$ along several lines. (a) The lines $L_{i}(i=1-5)$ are displayed: $L_{1}$, a straight line passing through the origin and making an angle of $3^{\circ}$ with the horizontal $\phi=0$ line. $L_{2}$, a straight line making an angle of $3^{\circ}$ with the $\phi=45^{\circ}$ line. $L_{3}$, a curved line whose equation is $\theta(\phi)=\arcsin [1 /(1$ $\left.\left.+\cos ^{2} \phi\right)\right]^{1 / 2}-3^{\circ} . L_{4}$, an arc of circle centered on $\bar{S}$ with a radius of $3^{\circ}$ (distances on this panel are measured in degrees). $L_{5}$, an arc of circle centered on $\bar{B}$ with a radius of $3^{\circ}$. See text for a description of the configurations associated with these lines. (b) $\bar{G}$ as a function of $\theta$ along line $L_{1}$. (c) $\bar{G}$ as a function of $\theta$ along line $L_{2}$. (d) $\bar{G}$ as a function of $\phi$ along line $L_{3}$. (e) $\bar{G}$ as a function of an angle $\alpha$, along line $L_{4}$. $\alpha=0$ corresponds to the point at which $L_{4}$ intercepts $\bar{C} \bar{S}$. (f) $\bar{G}$ as a function of an angle $\beta$, along line $L_{5}$. $\beta=0$ corresponds to the point at which $L_{5}$ intercepts $\bar{C} \bar{B}$. The contour lines displayed are the same as those in the bottom plane of Fig. 1(a). constant $\phi$, and the dashed circular lines to constant $\theta$. The solid lines on that bottom plane are contours of constant $\bar{G}$. Although these $\bar{G}$ are quadratic functions of $x(\theta, \phi)$, $y(\theta, \phi)$, and $z(\theta, \phi)$, the values of $\bar{G}^{4}{ }_{\Omega_{J_{\lambda}}}^{2} \Omega_{L_{\lambda}}^{1}{ }_{1}^{1}$ do not display significant oscillations over the allowed ranges of $\theta$ and $\phi$. In addition, the ranges of these $\bar{G}$ are rather small. It is interesting to analyze the behavior of the $\bar{G}$ functions near special configurations, namely coplanar, prolate symmetric top, oblate symmetric top, spherical top, and collinear configurations. For the symmetric and spherical top configurations the $\hat{\wedge}^{2}$ operator has poles, but the $\bar{G}$ functions should nevertheless have a regular behavior. This analysis is done in Fig. 3 for the $\bar{G}^{4}{ }_{1-1}^{1} 11$ function that was shown in Fig. 1(a). In panel 3(a) we display the bottom plane of Fig. 2(a), defined by Eqs. (6.5) and (6.6), in which contours of constant values of $\bar{G}$ are displayed. In addition, five dashed lines are shown: (1) $L_{1}$, a straight line passing through the origin and making an angle of $3^{\circ}$ with the horizontal $\phi=0$ line. $L_{1}$ is associated with configurations that are nearly coplanar. (2) $L_{2}$, a straight line making an angle of $3^{\circ}$ with the $\phi=45^{\circ}$ line. $L_{2}$ is associated with configurations that are nearly symmetric tops. (3) $L_{3}$, a curved line having the equation $\theta(\phi)=\arcsin \left[1 /\left(1+\cos ^{2} \phi\right)\right]^{1 / 2}-3^{\circ} . L_{3}$ represents configurations that are close to oblate symmetric top configurations. (4) $L_{4}$, an arc of circle centered on $\bar{S}$ with a radius $3^{\circ}$ (remember that $\theta$ is a polar radius measured in units of angle), corresponding to near-spherical-top configurations spanning the range from the prolate symmetric top (points near the $\bar{C} \bar{S}$ line) to the oblate symmetric top (points near the $\bar{C} \bar{B}$ line). (5) $L_{5}$, an arc of circle centered on $\mathrm{B}$ with a radius of $3^{\circ}$, corresponding to configurations near oblate-coplanar geometries. Panels 3(b)-3(f) depict the $\bar{G}_{1-1}^{4}{ }_{1}^{2} 1$ function along those five lines, respectively. The shape of all of those five curves is, as expected, very smooth and regular, with no unusual behavior, in spite of the fact that they sample geom- 
TABLE III. Principal-axes-of-inertia hyperspherical harmonics $G$ for $n$ $=4, J=2, L=2, D=2$.

\begin{tabular}{|c|c|c|c|}
\hline$\Omega_{J_{\lambda}}$ & $\Omega_{L_{\lambda}}$ & $G^{4} \underset{\Omega_{J_{\lambda}}^{2}}{2} \Omega_{L_{\lambda}}^{2} \underset{1}{2}=G^{4} \stackrel{2}{2} \Omega_{J_{\lambda}}-{\stackrel{2}{L_{L_{\lambda}}}}_{1}^{2}$ & $G^{4} \underset{\Omega_{J_{\lambda}}}{2} \Omega_{L_{\lambda}}^{2} \quad 2=G^{4}{ }_{-\Omega_{J_{\lambda}}}^{2}-\Omega_{L_{\lambda}}^{2}{ }^{2}$ \\
\hline 0 & 0 & $\begin{array}{c}-3\left(11 x^{4}+11 y^{4}-30 x^{2} y^{2}\right. \\
\left.+8 x^{2} z^{2}+8 y^{2} z^{2}-2 z^{4}\right)\end{array}$ & 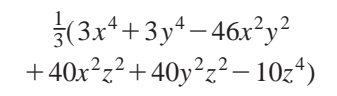 \\
\hline 0 & 2 & $2 \sqrt{6} x y\left(3 x^{2}+3 y^{2}+8 z^{2}\right)$ & $-2 \sqrt{\frac{2}{3}} x y\left(5 x^{2}+5 y^{2}-4 z^{2}\right)$ \\
\hline 1 & -1 & $-\frac{1}{2} y z\left(-127 x^{2}+107 y^{2}-5 z^{2}\right)$ & $\frac{1}{2} y z\left(-7 x^{2}+19^{2}+3 z^{2}\right)$ \\
\hline 1 & 1 & $-\frac{1}{2} x z\left(107 x^{2}-127 y^{2}-5 z^{2}\right)$ & $\frac{1}{2} x z\left(19 x^{2}-7 y^{2}+3 z^{2}\right)$ \\
\hline 2 & 0 & $2 \sqrt{6} x y\left(3 x^{2}+3 y^{2}+8 z^{2}\right)$ & $-2 \sqrt{\frac{2}{3}} x y\left(5 x^{2}+5 y^{2}-4 z^{2}\right)$ \\
\hline 2 & 2 & $x^{2}\left(5 x^{2}+31 y^{2}-56 z^{2}\right)$ & $x^{2}\left(3 x^{2}-23 y^{2}+8 z^{2}\right)$ \\
\hline \multicolumn{2}{|c|}{$N^{42} \underset{d}{2}$} & 0.2653046 & 1.2610633 \\
\hline
\end{tabular}

etries close to the special configurations considered, confirming the well-behaved nature of the $\bar{G}$ functions.

All of the $G$ functions for $n=4, J=2$, and $L=2$ are given in Table III. $D(4,2,2)$ is determined by our recursive procedure to be equal to 2 , and $n=4$ is the lowest value of $n$ for which we encountered degeneracy in these functions. By
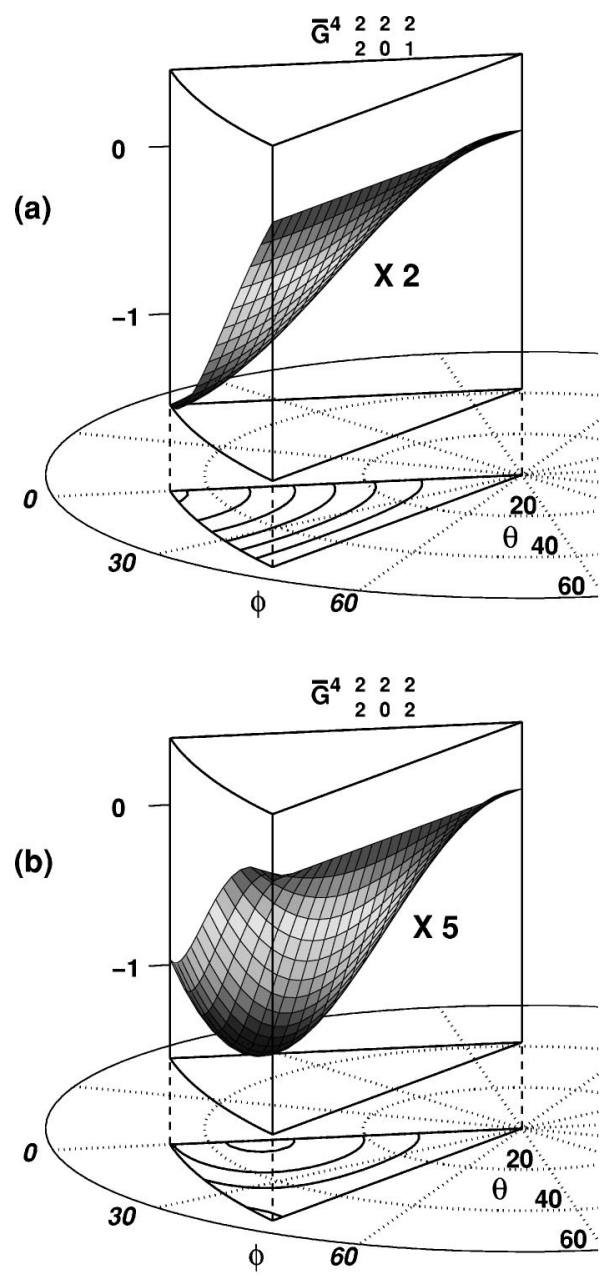

FIG. 4. The degenerate hyperspherical harmonics $\bar{G}^{4} 220 d$ as a function of $\theta$ and $\phi$. The meaning of the symbols is the same as for Fig. 2. (a) $d=1, \mathcal{N}$ $=0.2653$. Scaled contour values of -0.5 to 0 in steps of 0.25 . (b) $d=2$, $\mathcal{N}=1.2611$. Scaled values of -1.5 to 0 in steps of 0.5 .

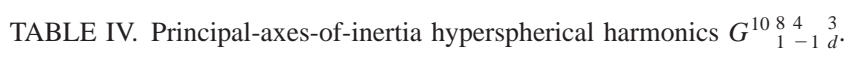

\begin{tabular}{|c|c|c|}
\hline$d$ & $G^{108} \begin{array}{lll}4 & 3 \\
1-1 & d\end{array}$ & $N^{10843}$ \\
\hline 1 & $\begin{array}{c}-\frac{1}{4 \sqrt{10010}} y z\left[36 x^{8}-2 x^{6}\left(51 y^{2}+155 z^{2}\right)\right. \\
+\left(y^{2}-z^{2}\right)^{2}\left(9 y^{4}-61 y^{2} z^{2}-4 z^{4}\right) \\
-6 x^{4}\left(55 y^{4}+258 y^{2} z^{2}-66 z^{4}\right) \\
\left.-x^{2}\left(33 y^{6}+1038 y^{4} z^{2}-1077 y^{2} z^{4}+118 z^{6}\right)\right]\end{array}$ & 762.439046 \\
\hline 2 & $\begin{array}{l}-\frac{1}{4 \sqrt{10010}} y z\left[1136 x^{8}-2 x^{6}\left(151 y^{2}+855 z^{2}\right)\right. \\
\quad+\left(y^{2}-z^{2}\right)^{2}\left(409 y^{4}+239 y^{2} z^{2}-4 z^{4}\right) \\
\quad+x^{4}\left(-3330 y^{4}+1152 y^{2} z^{2}+1296 z^{4}\right) \\
\left.+x^{2}\left(1667 y^{6}+162 y^{4} z^{2}+177 y^{2} z^{4}-718 z^{6}\right)\right]\end{array}$ & 155.108942 \\
\hline 3 & $\begin{array}{c}-\frac{1}{\sqrt{10010}} y z\left[177 x^{8}-4 x^{6}\left(66 y^{2}+25 z^{2}\right)\right. \\
+\left(y^{2}-z^{2}\right)^{2}\left(63 y^{4}+78 y^{2} z^{2}-8 z^{4}\right) \\
-x^{4}\left(960 y^{4}+111 y^{2} z^{2}+73 z^{4}\right) \\
\left.+x^{2}\left(144 y^{6}-166 y^{4} z^{2}+284 y^{2} z^{4}+4 z^{6}\right)\right]\end{array}$ & 211.111783 \\
\hline
\end{tabular}

contrast, for $J=L=0,{ }^{19}$ degeneracy is not encountered until the value $n=12$ is reached. The degenerate functions $\bar{G}^{4} \begin{array}{lll}2 & 2 & 2 \\ 0 & 1\end{array}$ and $\bar{G}_{2}^{4} 2202$ are plotted in Fig. 4. As mentioned in Sec. VC, these functions are not orthogonal to each other; nevertheless, they have quite different shapes, and it would be inappropriate to use one but not the other in a basis set expansion involving the $F$ functions.

\section{E. Hyperspherical harmonics for $n=10$}

As an example of hyperspherical harmonic $\bar{G}$ functions for higher $n$ and higher degeneracy, we give in Table IV the

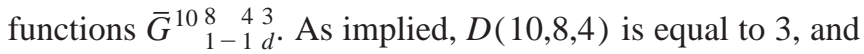
therefore $d$ can assume the values 1,2, and 3. Each of these three functions has 19 nonvanishing terms that are similar, only their coefficients being different. This is a pattern followed in general by degenerate hyperspherical harmonics $G$.

\section{F. Hyperspherical harmonics for $n=20$ and $\mathbf{3 0}$}

Even though the $\bar{G}^{n}{ }_{\Omega_{J_{\lambda}}}^{J}{ }_{\Omega_{L_{\lambda}}}^{L} \quad{ }^{D}$ are homogeneous polynomials of degree $n$ in $x(\theta, \phi), y(\theta, \phi)$, and $z(\theta, \phi)$, as $n$ increases to 30 they do not display pronounced oscillatious as a functions of $\theta$ and $\phi$. This is due to the limited ranges of these two angles, given by Eq. (2.9). As an example, the function $\bar{G}^{2084}{ }_{1-1}^{4}, \bar{G}^{201684}{ }_{1-1}, \bar{G}^{3084}{ }_{1-1}^{4}{ }_{1}$, and $\bar{G}^{3016828}{ }_{1-1}^{28}$ are depicted in Fig. 5. Even for the $n=30$ functions displayed in panels (c) and (d), there are fewer than four oscillations, and their amplitudes are not very pronounced. This suggests that the $\theta$ and $\phi$ Gauss-Legendre quadrature grids used in the evaluation of matrix elements involving these $\bar{G}$ functions need not be very fine.

\section{G. Hyperspherical harmonics for $J=L=n$}

The $G$ functions for the particular cases $n=J=L, \Omega_{J_{\lambda}}$ $= \pm n$, and $\Omega_{L_{\lambda}}= \pm n$ are very simple and can be obtained without resorting to the Mathematica program. As shown in Appendix F, they are given by 
(a)
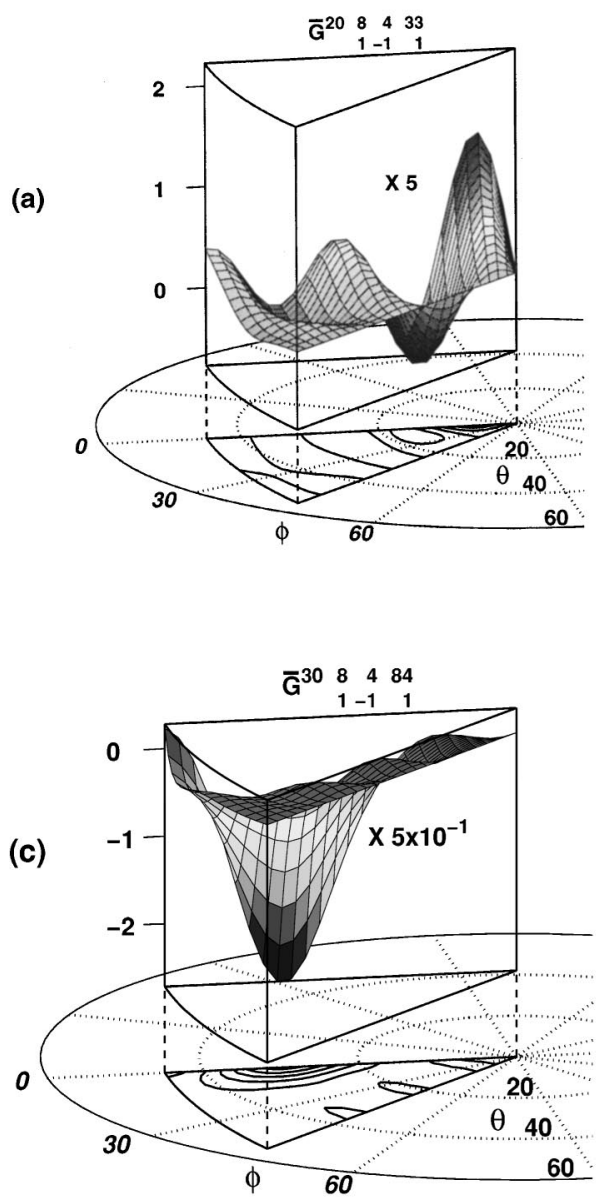

$$
G_{ \pm n \pm n d}^{n} \underset{ \pm}{n} D(\theta, \phi)=x^{n}
$$

and

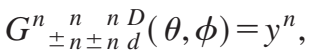

where $D(n, n, n)=1$. The $n=20$ and $n=30 \bar{G}$ functions, $\bar{G}^{2020201}, \bar{G}_{20201}^{20201}, \bar{G}_{30301}^{30301}$ and $\bar{G}_{3030301}^{303010}$ are displayed in Fig. 6. Each of these functions has a sharp spike, due to the large values of $n$ in Eqs. (6.7) and (6.8). Their value at these spikes is however small, and away from them their value is negligible. As a result, no special problems should be encountered in the numerical evaluation of matrix elements involving these kinds of $\bar{G}$ functions.

\section{DISCUSSION}

Once the $G^{n}{ }_{\Omega_{J_{\lambda}}^{J}}^{\Omega_{L_{\lambda}}} \stackrel{D}{d}$ d hyperspherical harmonics have been obtained, replacement into Eq. (3.18) furnishes the $F^{\Pi n J}{ }_{M_{J} M_{L_{\lambda}}^{L}}^{L}{ }_{d}^{D}\left(\chi, \Theta_{\lambda}\right)$ hyperspherical harmonics in ROHC. These can then be used as a basis set for expanding the local hyperspherical surface functions (LHSF) $\Phi$, which by definition are the eigenfunctions of the surface Hamiltonian,

$$
\hat{h}\left(\boldsymbol{\Theta}_{\lambda} ; \rho\right)=\frac{\hat{\wedge}^{2}\left(\boldsymbol{\Theta}_{\lambda}\right)}{2 \mu \rho^{2}}+V\left(\rho, \theta, \phi, \boldsymbol{\delta}_{\lambda}\right),
$$

where $V$ is the potential energy function described after Eq. (3.18). Once that expansion is performed, and the properties
FIG. 5. $\bar{G}^{n}$ for $n=20$ and $n=30$ as a function of $\theta$ and $\phi$. The meaning of the symbols is the same as for Fig. 2. The spacing of the scaled $\bar{G}$ contour lines in all panels is 0.5. (a)

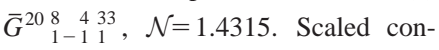
tours from -0.5 to 1.0 . (b) $\bar{G}^{20} 1688$, $\mathcal{N}=15336.6517$. Scaled contours from -0.5 to 2.0 . (c) $\bar{G}_{1-11}^{308}, \mathcal{N}$ $=1.6283$. Scaled contours from -2.5 to $0 . \quad$ (d) $\bar{G}^{3016828} 1-11, \quad \mathcal{N}=6.2019$. Scaled contours from -1.5 to 1.0 . of the Wigner rotation functions appearing in Eq. (3.18) are taken into account, the corresponding coefficients must satisfy, for each $\Pi$ and $J$, a generalized eigenvalue-eigenvector equation involving matrices that are independent of $M_{J}$ and whose rows and columns are spanned by the five quantum numbers $n_{\Pi}, L, M_{L_{\lambda}}, D$, and $d$. The calculation of these matrices involves quadratures over the five variables $\delta_{\lambda}^{(1)}$, $\delta_{\lambda}^{(2)}, \delta_{\lambda}^{(3)}, \theta$, and $\phi$ on which $V\left(\rho, \boldsymbol{\delta}_{\lambda}, \theta, \phi\right), \bar{G}^{n}{ }_{\Omega_{J_{\lambda}}} \Omega_{L_{\lambda}}^{L}{ }_{d}^{D}$, and $D_{\Omega_{L_{\lambda}} M_{L_{\lambda}}}^{L}\left(\boldsymbol{\delta}_{\lambda}\right)$ depend. Since all these functions are now known and since the $V$ and $G$ functions do not vary very rapidly with $\boldsymbol{\delta}_{\lambda}, \theta$, and $\phi$, these quadratures can be performed in large blocks simultaneously, so as to minimize the duplication of numerical operations and thereby optimize the corresponding computer time. We have developed a method in which the average time to compute one matrix element scales approximately as the total number of two-dimensional grid points in the $\theta, \phi$ quadratures rather than as the total number of five-dimensional points (i.e., the product of the number of points for each dimension). ${ }^{30}$ The quadrature time needed to compute those matrices is, as usual, proportional to the square of the total number $\omega$ of sets of values of $n_{\Pi}$, $L, M_{L_{\lambda}}, D$, and $d$ needed to achieve convergence, but the coefficient of $\omega^{2}$ is much smaller in our method than if the quadratures were not performed in large blocks, according to the strategy indicated.

Since all Coriolis terms are incorporated in the $G$ func- 

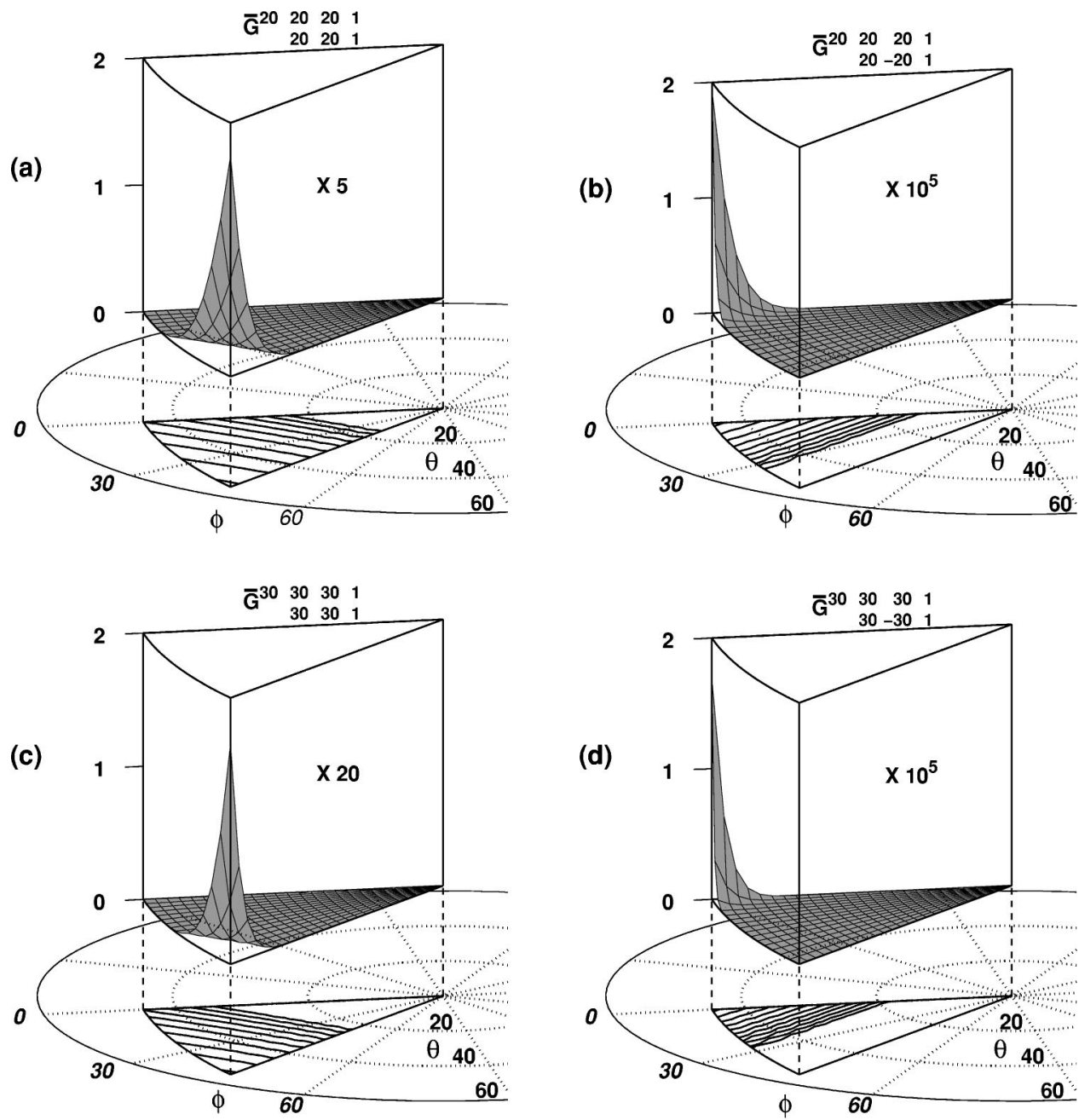

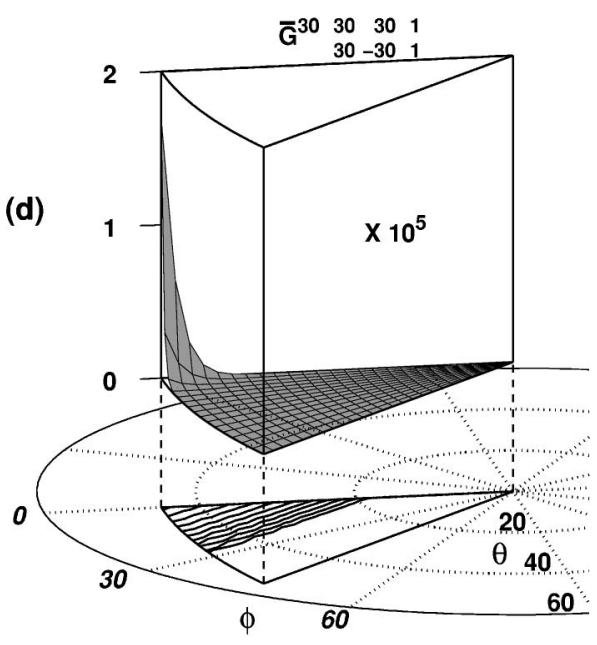

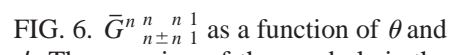
$\phi$. The meaning of the symbols is the same as for Fig. 2. The scaled $\bar{G}$ contour values, for all panels, vary from $10^{-9}$ to 1 in factors of 10 . (a) and (b): $n=20, \mathcal{N}=20525.3861$. (c) and (d): $n=30, \mathcal{N}=1187768.9$. tions, all the couplings in the equations satisfied by the coefficients of the LHSF expansion are potential function couplings. Furthermore, the equations for $\Pi=0$ (i.e., even values of $n$ ) are decoupled from those for $\Pi=1$ (i.e., odd values of $n$ ). In addition, as mentioned at the end of Sec. III, $F$ hyperspherical harmonics for $\Gamma$ irreducible representations of the permutation groups of identical atoms the system of interest may contain can easily be generated. Using such parity and irreducible representation $F$ functions decreases the numerical effort required to generate the corresponding LHSF.

Due to the first term in the right-hand side of Eq. (7.1) a hypercentrifugal potential matrix, diagonal in $n_{\Pi}$, will appear as an additive term in the matrix whose generalized eigenvalues and eigenvectors must be evaluated. The diagonal terms of that hypercentrifugal matrix are $n_{\Pi}\left(n_{\Pi}\right.$ $+7) \hbar^{2} / 2 \mu \rho^{2}$. For a given $J$, Eq. (3.12) requires that $n_{\Pi}$ $\geqslant J$. On the other hand, due to the highly repulsive nature of the hypercentrifugal matrix elements, it is expected that the the rate of convergence of the calculation with respect to $n_{\Pi}$ will be high, i.e., that values of $n_{\Pi}$ much larger than $J$ will not be needed.

We plan to use this methodology to calculate state-tostate integral and differential cross sections of benchmark quality for the $\mathrm{OH}+\mathrm{H}_{2} \rightarrow \mathrm{H}_{2} \mathrm{O}+\mathrm{H}$ reaction. We estimate that values of $J$ from 0 through 25 will be required for conver- gence of the calculations for ground state relative reagent translational energies of $0.7 \mathrm{eV} .^{31} \mathrm{We}$ also estimate, based on quasiclassical trajectory calculations of the range of the dynamically active rotational quantum numbers of the $\mathrm{H}_{2} \mathrm{O}$ molecule, ${ }^{32}$ that the number of propagation channels for each $\Pi$ and $J$ will be of the order of 10000 before decoupling the irreducible representations $\Gamma$ of the permutation group $P_{3}$ of the three identical $\mathrm{H}$ atoms, and 5000 after that decoupling is performed. In other words, the size of the matrices involved in the calculations should be about $5000 \times 5000$ and, for each $\rho, \Gamma, \Pi$, and $J$, we need to calculate in general around 5000 accurate surface functions. This requires that in the expansion described above, the quantity $\omega$ should equal about 10000 , in order to result in 5000 surface functions that are accurate enough to be used in the propagation equations. Our estimate is that all the associated quadratures involving all the $F$ functions needed to calculate the LHSF at 30 values of $\rho$ from 2 to about 8 bohr (the range of the strong interaction region), using the methods mentioned, will take about $9 \mathrm{~h}$ on a parallel computer having a sustained speed of 100 Gflops for these calculations, such as the HP V2500 machine at Caltech. In addition, we must calculate eigensolutions for each of these values of $\rho$. Between $8 \mathrm{bohr}$ and $14 \mathrm{bohr}$ approximately (the weak interaction region, where the reagent and product channels overlap only slightly), Delves hyperspherical coordinates will be used ${ }^{33,34}$ and require an equiva- 
lent amount of computer time. Finally, we must perform propagations for each $\Gamma, \Pi, J$ and energy. These propagations, using a logarithmic derivative method, ${ }^{4}$ will consume a majority of the computer time required for the cross section evaluations. This indicates that the basis set of hyperspherical harmonic functions described and obtained in the present paper have the potential for generating all the matrix elements needed, in an acceptable amount of computer time, to calculate all the strong interaction LHSF needed for benchmark-quality calculations of the cross sections described above. Furthermore, in spite of the large number of five-dimensional quadratures involved, this computational effort is a small fraction of the total computational time required for obtaining those state-to-state cross sections. The latter is estimated, for 20 energies, to be about $550 \mathrm{~h}$ on that computer. This is an acceptable amount of computer time, given the importance of performing benchmark-quality calculations for at least one tetraatomic chemical reaction and the fact that parallel computers in the Tflops range, and on which that computer time would be an order of magnitude or more smaller, are already available.

\section{SUMMARY AND CONCLUSIONS}

We have described and implemented a recursive procedure for generating analytical hyperspherical harmonics for tetraatomic systems in row-orthonomal hyperspherical coordinates. The implementation was performed with a Mathematica algebraic program and used to generate all such functions for values of the hyperangular momentum quantum $n$ up to 30 . About 43.8 million of such functions were generated. It was also indicated how such large number of functions can be used in practice to solve reactive scattering problems for these systems on currently available high performance computers. The hyperspherical harmonics obtained are shown to be correct by verifying that they satisfy the appropriate coupled partial differential equations. They agree with the small number of such functions calculated previously for $n=1$ and 2 and all $J$ and $L,{ }^{22}$ and, for $n=1-7$, for $J=L=0 .{ }^{19}$ The degeneracy of these hyperspherical harmonics was also calculated, and achieved a maximum value of $D=127$ for $n=30, J=14$, and $L=14$. It is argued that these functions are attractive candidates for benchmark-quality state-to-state reactive scattering calculations for these systems.

\section{ACKNOWLEDGMENTS}

This work has been supported in part by NSF Grant No. CHE 9810050.

\section{APPENDIX A: COUPLED PARTIAL DIFFERENTIAL EQUATIONS FOR THE TWO-ANGLE PRINCIPAL-AXES-OF-INERTIA HYPERSPHERICAL HARMONICS $G$}

The $G^{\Pi n_{\Pi}}{ }_{\Omega_{J_{\lambda}}}^{J}{ }_{L_{L_{\lambda}}}^{L}{ }_{d}^{D}(\theta, \phi)$ hyperspherical harmonics have been defined by Eq. (3.18). As justified after Eq. (3.19), the ranges of the $\boldsymbol{\delta}_{\lambda}$ angles in these equations can be taken to be those given by Eq. (2.8) or, instead, by Eq. (3.19). The

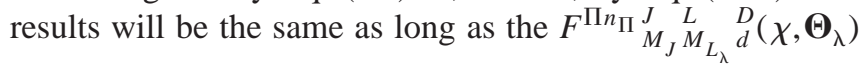

are the same at the four sets of $\left(\boldsymbol{\Theta}_{\lambda}\right)_{i}$ angles defined by Eqs. (3.21)-(3.24). We will impose this constraint throughout this paper, and as a result, we are allowed to use the range of the $\boldsymbol{\delta}_{\boldsymbol{\lambda}}$ given by Eq. (3.19) whenever desired. We will do so in

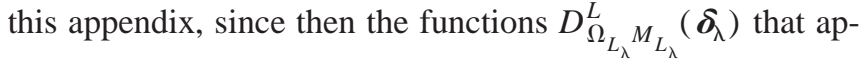
pear in Eq. (3.18) are orthogonal. Under these conditions, the coupled partial differential equations satisfied by the $G^{\Pi n_{\Pi}}{ }_{\Omega_{J_{\lambda}}}^{J}{ }_{\Omega_{L_{\lambda}}}{ }_{d}^{D}(\theta, \phi)$ can be obtained by standard methods. To that effect, we remember that

$$
\hat{J}^{2}=\hat{J}_{1}^{I_{\lambda}^{2}}+\hat{J}_{2}^{I_{\lambda}^{2}}+\hat{J}_{2}^{I_{\lambda}^{2}}
$$

and

$$
\hat{L}^{2}=\hat{L}_{\lambda_{1}}^{2}+\hat{L}_{\lambda_{2}}^{2}+\hat{L}_{\lambda_{3}}^{2}
$$

and define the operators

$$
\hat{J}_{ \pm}^{I_{\lambda}}=\hat{J}_{1}^{I_{\lambda}} \pm i \hat{J}_{2}^{I_{\lambda}}
$$

and

$$
\hat{L}_{\lambda \pm}=\hat{L}_{\lambda_{1}} \pm i \hat{L}_{\lambda_{2}}
$$

where the $\hat{J}_{i}^{I_{\lambda}}$ and $\hat{L}_{\lambda_{i}}$ are given explicitly by Eqs. (2.25) and (2.26), respectively. As a result, we can express $\hat{J}_{i}^{I_{\lambda}}, \hat{J}_{i}^{I_{\lambda}^{2}}, \hat{L}_{\lambda_{i}}$, and $\hat{L}_{\lambda_{i}}^{2}$ in terms of the $\hat{J}_{ \pm}^{I_{\lambda}}$ and $\hat{L}_{\lambda \pm}$ as

$$
\begin{aligned}
& \hat{J}_{1}^{I_{\lambda}}=\frac{1}{2}\left(\hat{J}_{+}^{I_{\lambda}}+\hat{J}_{-}^{I_{\lambda}}\right), \\
& \hat{J}_{2}^{I_{\lambda}}=\frac{1}{2 i}\left(\hat{J}_{+}^{I_{\lambda}}-\hat{J}_{-}^{I_{\lambda}}\right), \\
& \hat{J}_{1}^{I_{\lambda}^{2}}=\frac{1}{2}\left(\hat{J}^{2}-\hat{J}_{3}^{I_{\lambda}^{2}}\right)+\frac{1}{4}\left(\hat{J}_{+}^{I_{\lambda}^{2}}+\hat{J}_{-}^{I_{\lambda}^{2}}\right), \\
& \hat{J}_{2}^{I_{\lambda}^{2}}=\frac{1}{2}\left(\hat{J}^{2}-\hat{J}_{3}^{I_{\lambda}^{2}}\right)-\frac{1}{4}\left(\hat{J}_{+}^{2}+\hat{J}_{-}^{I_{\lambda}^{2}}\right), \\
& \hat{L}_{\lambda_{1}}=\frac{1}{2}\left(\hat{L}_{\lambda+}+\hat{L}_{\lambda-}\right), \\
& \hat{L}_{\lambda_{2}}=\frac{1}{2 i}\left(\hat{L}_{\lambda+}-\hat{L}_{\lambda-}\right), \\
& \hat{L}_{\lambda_{1}}^{2}=\frac{1}{2}\left(\hat{L}^{2}-\hat{L}_{\lambda_{3}}^{2}\right)+\frac{1}{4}\left(\hat{L}_{\lambda+}^{2}+\hat{L}_{\lambda-}^{2}\right), \\
& \hat{L}_{\lambda_{2}}^{2}=\frac{1}{2}\left(\hat{L}^{2}-\hat{L}_{\lambda_{3}}^{2}\right)-\frac{1}{4}\left(\hat{L}_{\lambda+}^{2}+\hat{L}_{\lambda-}^{2}\right) .
\end{aligned}
$$

We now expand the terms in $\left(N_{i i} \hat{J}_{k}^{I_{\lambda}}-N_{j j} \hat{L}_{\lambda_{k}}\right)^{2}$ in the expression for the $\hat{C}^{2}$ operator given by Eq. (2.22) and use Eqs. (A1)-(A12) to express that operator, and therefore $\hat{\wedge}^{2}$, in terms of $\hat{J}^{2}, \hat{J}_{3}^{I_{\lambda}}, \hat{J}_{ \pm}^{I_{\lambda}}, \hat{L}^{2}, \hat{L}_{\lambda_{3}}$, and $\hat{L}_{\lambda_{ \pm}}$. We then replace Eq. (3.18) into Eq. (3.6). To apply the several angular momentum operators in the resulting expression to the Wigner rotation functions, we remember that the $\hat{J}_{i}^{I_{\lambda}}$ operators are 
body-fixed, whereas the $\hat{L}_{\lambda_{k}}$ are of the space-fixed-type variety. The effect of these operators on those Wigner rotation functions are $^{25}$

$$
\begin{aligned}
& \hat{J}^{2} D_{M_{J} \Omega_{J_{\lambda}}}^{J}\left(\mathbf{a}_{\lambda}\right)=J(J+1) \hbar^{2} D_{M_{J} \Omega_{J_{\lambda}}}^{J}\left(\mathbf{a}_{\lambda}\right), \\
& \hat{J}_{3}^{I_{\lambda}} D_{M_{J} \Omega_{J_{\lambda}}}^{J}\left(\mathbf{a}_{\lambda}\right)=\Omega_{J_{\lambda}} \hbar D_{M_{J} \Omega_{J_{\lambda}}}^{J}\left(\mathbf{a}_{\lambda}\right), \\
& \hat{J}_{ \pm}^{I_{\lambda}} D_{M_{J} \Omega_{J_{\lambda}}}^{J}\left(\mathbf{a}_{\lambda}\right)=\hbar \xi_{\mp}\left(J, \Omega_{J_{\lambda}}\right) D_{M_{J} \Omega_{J_{\lambda}} \mp 1}^{J}\left(\mathbf{a}_{\lambda}\right), \\
& \hat{L}^{2} D_{\Omega_{L_{\lambda}} M_{L_{\lambda}}}^{L}\left(\boldsymbol{\delta}_{\lambda}\right)=L(L+1) \hbar^{2} D_{\Omega_{L_{\lambda}} M_{L_{\lambda}}}\left(\boldsymbol{\delta}_{\lambda}\right), \\
& \hat{L}_{\lambda_{3}} D_{\Omega_{L_{\lambda}}^{L} M_{L_{\lambda}}}^{L}\left(\boldsymbol{\delta}_{\lambda}\right)=\Omega_{L_{\lambda}} \hbar D_{\Omega_{L_{\lambda}} M_{L_{\lambda}}}^{L}\left(\boldsymbol{\delta}_{\lambda}\right), \\
& \hat{L}_{\lambda \pm} D_{\Omega_{L_{\lambda}}^{L} M_{L_{\lambda}}}^{L}\left(\boldsymbol{\delta}_{\lambda}\right)=-\hbar \xi_{ \pm}\left(J, \Omega_{L_{\lambda}}\right) D_{\Omega_{L_{\lambda}} \pm 1 M_{L_{\lambda}}}^{L}\left(\boldsymbol{\delta}_{\lambda}\right),
\end{aligned}
$$

where

$$
\begin{aligned}
\xi_{ \pm}(j, \Omega) & =[j(j+1)-\Omega(\Omega \pm 1)]^{1 / 2} \\
& =[(j \mp \Omega)(j \pm \Omega+1)]^{1 / 2}
\end{aligned}
$$

with

$$
-j \leqslant \Omega \leqslant j \quad j \geqslant 0, \quad \text { integer. }
$$

Using Eqs. (A13)-(A18) together with the orthogonality of the Wigner rotation functions, we finally get the following system of coupled partial differential equations that must be satisfied by the $G^{n} \Pi_{\Omega_{J_{\lambda}}}^{J} \Omega_{L_{\lambda}}{ }_{d} d$ :

$$
\begin{aligned}
& {\left[\hat{K}^{2}(\theta, \phi)+\hat{B}(\theta, \phi)\right] G^{n} \prod_{\Omega_{J_{\lambda}}}^{J} \stackrel{L}{\Omega_{L_{\lambda}}} \quad \stackrel{D}{d}}
\end{aligned}
$$

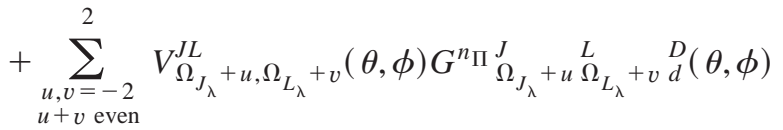

$$
\begin{aligned}
& =n(n+7) \hbar^{2} G^{n} \Pi \underset{\Omega_{J_{\lambda}}^{J}}{\Omega_{L_{\lambda}}^{L}} \stackrel{D}{d}, \\
& \Omega_{J_{\lambda}}=-J,-J+1, \ldots, J, \quad \Omega_{L_{\lambda}}=-L,-L+1, \ldots, L .
\end{aligned}
$$

The sum over $u$ and $v$ is limited to terms for which $u$ $+v$ is even, i.e., to the nine $(u, v)$ pairs $(0,0),(1,1),(-1$, $-1),(1,-1),(-1,1),(2,0),(-2,0),(0,2)$ and $(0,-2)$. The corresponding $V_{\Omega_{J_{\lambda}}+u, \Omega_{L_{\lambda}}{ }^{+} \text {v }}$ are effective potentials that have poles at the same configurations for which $\hat{C}^{2}$ has poles and are

(a) $u=v=0$,

$$
\begin{aligned}
V_{\Omega_{J_{\lambda}}, \Omega_{L_{\lambda}}}^{J L}(\theta, \phi)= & \hbar^{2}[J(J+1)+L(L+1)] \\
& \times\left[\frac{N_{22}^{2}+N_{33}^{2}}{2\left(N_{22}^{2}-N_{33}^{2}\right)^{2}}+\frac{N_{11}^{2}+N_{33}^{2}}{2\left(N_{11}^{2}-N_{33}^{2}\right)^{2}}\right] \\
& +\hbar^{2}\left(\Omega_{J_{\lambda}}^{2}+\Omega_{L_{\lambda}}^{2}\right)\left[\frac{N_{11}^{2}+N_{22}^{2}}{\left(N_{11}^{2}-N_{22}^{2}\right)^{2}}\right. \\
& \left.-\frac{N_{11}^{2}+N_{33}^{2}}{2\left(N_{11}^{2}-N_{33}^{2}\right)^{2}}-\frac{N_{22}^{2}+N_{33}^{2}}{2\left(N_{22}^{2}-N_{33}^{2}\right)^{2}}\right] \\
& -4 \hbar^{2} \Omega_{J_{\lambda}} \Omega_{L_{\lambda}} \frac{N_{11} N_{22}}{\left(N_{11}^{2}-N_{22}^{2}\right)^{2}}
\end{aligned}
$$

(b) $u=v=1$,

$$
\begin{aligned}
V_{\Omega_{J_{\lambda}}+1, \Omega_{L_{\lambda}}+1}^{J L}(\theta, \phi)= & \hbar^{2} \xi_{-}\left(J, \Omega_{J_{\lambda}}+1\right) \\
& \times \xi_{-}\left(L, \Omega_{L_{\lambda}}+1\right) f_{1}(\theta, \phi),
\end{aligned}
$$

where

$$
f_{1}(\theta, \phi)=\frac{N_{11} N_{33}}{\left(N_{11}^{2}-N_{33}^{2}\right)^{2}}+\frac{N_{22} N_{33}}{\left(N_{22}^{2}-N_{33}^{2}\right)^{2}} ;
$$

(c) $u=v=-1$,

$$
\begin{aligned}
V_{\Omega_{J_{\lambda}}-1, \Omega_{L_{\lambda}}-1}^{J L}(\theta, \phi)= & \hbar^{2} \xi_{+}\left(J, \Omega_{J_{\lambda}}-1\right) \\
& \times \xi_{+}\left(L, \Omega_{L_{\lambda}}-1\right) f_{1}(\theta, \phi) ;
\end{aligned}
$$

(d) $u=1, v=-1$,

$$
\begin{aligned}
V_{\Omega_{J_{\lambda}}+1, \Omega_{L_{\lambda}}-1}^{J L}(\theta, \phi)= & \hbar^{2} \xi_{-}\left(J, \Omega_{J_{\lambda}}+1\right) \\
& \times \xi_{+}\left(L, \Omega_{L_{\lambda}}-1\right) f_{2}(\theta, \phi),
\end{aligned}
$$

where

$$
f_{2}(\theta, \phi)=\frac{N_{22} N_{33}}{\left(N_{22}^{2}-N_{33}^{2}\right)^{2}}-\frac{N_{11} N_{33}}{\left(N_{11}^{2}-N_{33}^{2}\right)^{2}} ;
$$

(e) $u=-1, v=1$,

$$
\begin{aligned}
V_{\Omega_{J_{\lambda}}-1, \Omega_{L_{\lambda}}+1}^{J L}(\theta, \phi)= & \hbar^{2} \xi_{+}\left(J, \Omega_{J_{\lambda}}-1\right) \\
& \times \xi_{-}\left(L, \Omega_{L_{\lambda}}+1\right) f_{2}(\theta, \phi) ;
\end{aligned}
$$

(f) $u=2, v=0$,

$$
\begin{aligned}
V_{\Omega_{J_{\lambda}}+2, \Omega_{L_{\lambda}}}^{J L}(\theta, \phi)= & \hbar^{2} \xi_{-}\left(J, \Omega_{J_{\lambda}}+2\right) \\
& \times \xi_{-}\left(J, \Omega_{J_{\lambda}}+1\right) f_{3}(\theta, \phi),
\end{aligned}
$$

where

$$
\begin{aligned}
& f_{3}(\theta, \phi)=\frac{1}{4}\left[\frac{N_{22}^{2}+N_{33}^{2}}{\left(N_{22}^{2}-N_{33}^{2}\right)^{2}}-\frac{N_{11}^{2}+N_{33}^{2}}{\left(N_{11}^{2}-N_{33}^{2}\right)^{2}}\right] ; \\
& (\mathrm{g}) u=-2, v=0, \\
& V_{\Omega_{J_{\lambda}} L_{2, \Omega_{L_{\lambda}}}(\theta, \phi)=} \hbar^{2} \xi_{+}\left(J, \Omega_{J_{\lambda}}-2\right) \\
& \quad \times \xi_{+}\left(J, \Omega_{J_{\lambda}}-1\right) f_{3}(\theta, \phi) ;
\end{aligned}
$$


(h) $u=0, v=2$,

$$
\begin{aligned}
V_{\Omega_{J_{\lambda}}, \Omega_{L_{\lambda}}+2}^{J L}(\theta, \phi)= & \hbar^{2} \xi_{-}\left(L, \Omega_{L_{\lambda}}+2\right) \\
& \times \xi_{-}\left(L, \Omega_{L_{\lambda}}+1\right) f_{3}(\theta, \phi) ;
\end{aligned}
$$

(i) $u=0, v=2$,

$$
\begin{aligned}
V_{\Omega_{J_{\lambda}}, \Omega_{L_{\lambda}}-2}^{J L}(\theta, \phi)= & \hbar^{2} \xi_{+}\left(L, \Omega_{L_{\lambda}}-2\right) \\
& \times \xi_{+}\left(L, \Omega_{L_{\lambda}}-1\right) f_{3}(\theta, \phi) .
\end{aligned}
$$

It should be noticed that in Eq. (A21), $\Pi, n_{\Pi}, J$, and $L$ are fixed, whereas the $\Omega_{J_{\lambda}}$ and $\Omega_{L_{\lambda}}$ span the ranges indicated in Eq. (A22). The only differential operator appearing in Eq. (A21) is $\hat{K}^{2}+\hat{B}^{2}$ and it is diagonal, i.e., only acts on the $G$ having $u=v=0$. The entire coupling between $G$ functions is provided by the effective potentials $V_{\Omega_{J_{\lambda}}+u, \Omega_{L_{\lambda}}+v}(\theta, \phi)$ [which are unrelated to the potential energy function $\left.V\left(\rho, \theta, \phi, \boldsymbol{\delta}_{\lambda}\right)\right]$ for $u \neq 0$ and $v \neq 0$ and is completely due to the Coriolis coupling terms associated with the products of $\xi$ coefficients. As a result, if the $F^{\Pi n}{ }_{\Pi}^{J}{ }_{M_{J}}^{J} M_{L_{\lambda}}^{L} d\left(\chi, \boldsymbol{\Theta}_{\lambda}\right)$ functions of Eq. (3.18) are used to expand the LHSF which are, as mentioned in Sec. VII, the eigenfunctions of the local hyperspherical surface operator $\hat{h}$ defined by Eq. (7.1). The latter depends on $\rho$ only parametrically, i.e., does not contain differential operators in this variable $\rho$. The resulting algebraic equations in the coefficients of the this expansion will not contain any Coriolis coupling terms. It should also be noticed that Eq. (A21), in addition to not containing $M_{J}$ or $M_{L_{\lambda}}$, is invariant under the interchange of the $\left(J, \Omega_{J_{\lambda}}\right)$ and $\left(L, \Omega_{L_{\lambda}}\right)$ pairs. As a result, we have omitted $M_{J}$ and $M_{L_{\lambda}}$ from the indices specifying the $G$ functions and, in addition, the symmetry relation

$$
G^{n} \Omega_{J_{\lambda}}^{J} \stackrel{\Omega_{L_{\lambda}}}{L} \quad \stackrel{D}{d}(\theta, \phi)=G^{n} \Omega_{L_{\lambda}}^{L} \Omega_{J_{\lambda}}^{J} \quad d(\theta, \phi)
$$

has been derived. This property greatly decreases the number of independent $G$ functions that must be evaluated.

Finally, we remark that we do not attempt in this paper to solve Eq. (A21). Instead, we derive a recursion relation among the $G$ functions for consecutive values of $n$, based on the general properties of harmonic polynomials. We then use this recursion relation to analytically generate the $G$ functions. Equation (A21) is however used to check the correctness of the $G$ functions thus obtained.

\section{APPENDIX B: DETERMINATION OF THE CHIRALITY FUNCTION}

In order to determine the chirality function $f^{\Pi}(\chi)$ appearing in Eq. (3.16), we replace the latter into Eq. (3.11). This leads to

$$
f^{\Pi}(\chi+1)=(-1)^{\Pi} f^{\Pi}(\chi)
$$

from which we get

$$
f^{\Pi}(1)=(-1)^{\Pi} f^{\Pi}(0)
$$

and therefore

$$
\left|f^{\Pi}(1)\right|=\left|f^{\Pi}(0)\right| .
$$

We are free to require that $f^{\Pi}(\chi)$ be real and to have an absolute value of 1 . Therefore, we can set

$$
f^{\Pi}(\chi)=(-1)^{g(\chi)},
$$

where $g(\chi)$ is a function of $\chi$ that can only assume nonnegative integer values. Since, for a positive integer $i$, we have $\chi^{i}=\chi$, an expansion of $g(\chi)$ in a power series in $\chi$ leads to the solution

$$
g(\chi)=\alpha \chi,
$$

where $\alpha$ is a non-negative constant integer. Replacement of this result first into Eq. (B4) for $\chi=0,1$ and then into Eq. (B2) gives

$$
(-1)^{\alpha}=(-1)^{\Pi} .
$$

Therefore,

$$
\alpha=\Pi+2 m \quad m>0 \quad \text { integer }
$$

and

$$
f^{\Pi}(\chi)=(-1)^{(\Pi+2 m) \chi} .
$$

On the other hand, $f^{\Pi}(\chi)$ can at most depend on the quantum numbers that appear in $F$, namely, $n, J, M_{J}, L, M_{L_{\lambda}}$, $\Pi, D$, and $d$ but should not depend on any additional quantities. As a result, the non-negative integer $m$ in Eq. (B7) cannot be arbitrary, but must be determined by those quantum numbers. A convenient choice is to require that

$$
\Pi+2 m=n,
$$

where $n$ is the hyperangular momentum quantum number of Eq. (3.6). With this choice we get

$$
f^{\Pi}(\chi)=(-1)^{n \chi}=(-1)^{\Pi \chi} .
$$

As a result of Eq. (B9), $n$ is restricted to even values for $\Pi=0$ and odd ones for $\Pi=1$. In view of this restriction, we add a subscript $\Pi$ to $n$ and designate it by $n_{\Pi}$.

\section{APPENDIX C: LAPLACIAN IN COMPLEX $t_{\lambda}{ }^{k}$ VARIABLES}

In this appendix we derive an expression for the system's Laplacian in the complex variables defined by Eqs. (4.14)-(4.18). To that effect, let us define the column vectors $\mathbf{x}_{\lambda}$ and $\mathbf{t}_{\lambda}$ through their transposes as

$$
\widetilde{\mathbf{x}}_{\lambda}=\left(x_{\lambda_{1}}^{(1)}, x_{\lambda_{2}}^{(1)}, x_{\lambda_{3}}^{(1)}, x_{\lambda_{1}}^{(2)}, x_{\lambda_{2}}^{(2)}, x_{\lambda_{3}}^{(2)}, x_{\lambda_{1}}^{(3)}, x_{\lambda_{2}}^{(3)}, x_{\lambda_{3}}^{(3)}\right)
$$

and

$$
\widetilde{\mathbf{t}}_{\lambda}=\left(t_{\lambda 1}{ }_{1}^{1}, t_{\lambda 0}, t_{\lambda-1}^{1}, t_{\lambda 1}{ }_{1}^{0}, t_{\lambda}{ }_{0}^{0}, t_{\lambda-1}, t_{\lambda 1}^{-1}, t_{\lambda 0}^{-1}, t_{\lambda-1}^{-1}\right) .
$$

Equations (4.5)-(4.13) can be written in vector-matrix form as

$$
\mathbf{x}_{\lambda}=\mathbf{M t}_{\lambda}
$$

where $\mathbf{M}$ is a $9 \times 9$ matrix whose elements are either real or pure imaginary constants. It is straightforward to show that this matrix is unitary (but not orthogonal),

$\mathbf{M}^{\dagger} \mathbf{M}=\mathbf{I}$.

The inverses of Eqs. (4.5)-(4.13) are 


$$
\begin{aligned}
& t_{\lambda 1}^{1}=\frac{1}{2}\left(x_{\lambda_{1}}^{(1)}-i x_{\lambda_{2}}^{(1)}+i x_{\lambda_{1}}^{(2)}+x_{\lambda_{2}}^{(2)}\right), \\
& t_{\lambda 0}^{1}=\frac{1}{\sqrt{2}}\left(x_{\lambda_{1}}^{(3)}+i x_{\lambda_{2}}^{(3)}\right), \\
& t_{\lambda-1}^{1}=\frac{1}{2}\left(-x_{\lambda_{1}}^{(1)}-i x_{\lambda_{2}}^{(1)}-i x_{\lambda_{1}}^{(2)}+x_{\lambda_{2}}^{(2)}\right), \\
& t_{\lambda 1}^{0}=\frac{1}{\sqrt{2}}\left(x_{\lambda_{3}}^{(1)}-i x_{\lambda_{3}}^{(2)}\right), \\
& t_{\lambda{ }_{0}^{0}}=x_{\lambda_{3}}^{(3)}, \\
& t_{\lambda-1}^{0}=-\frac{1}{\sqrt{2}}\left(x_{\lambda_{3}}^{(1)}+i x_{\lambda_{3}}^{(2)}\right), \\
& t_{\lambda}^{-1}{ }_{1}=\frac{1}{2}\left(-x_{\lambda_{1}}^{(1)}+i x_{\lambda_{2}}^{(1)}+i x_{\lambda_{1}}^{(2)}+x_{\lambda_{2}}^{(2)}\right), \\
& t_{\lambda-1}^{-1}=\frac{1}{2}\left(x_{\lambda_{1}}^{(1)}+i x_{\lambda_{2}}^{(1)}-i x_{\lambda_{1}}^{(2)}+x_{\lambda_{2}}^{(2)}\right), \\
& \sqrt{2}\left(-x_{\lambda_{1}}^{(3)}+i x_{\lambda_{2}}^{(3)}\right),
\end{aligned}
$$

which in vector-matrix form are

$$
\mathbf{t}_{\lambda}=\mathbf{M}^{-1} \mathbf{x}_{\lambda} \text {. }
$$

Examination of these equations shows that

$$
t_{\lambda-j}^{-k}=(-1)^{j+k}\left(t_{\lambda j}^{k}\right)^{*} \text {. }
$$

In order to be able to express the Laplacian in the $t_{\lambda j}{ }_{j}^{k}$ variables, it is necessary to define the $\partial / \partial t_{\lambda j}{ }^{k}$ operators with $t_{\lambda j}{ }^{k}$ complex. We set

$$
\frac{\partial}{\partial t_{\lambda j}^{k}} \equiv \sum_{r, s=1}^{3} \frac{\partial x_{\lambda_{s}}^{(r)}}{\partial t_{\lambda j}^{k}} \frac{\partial}{\partial x_{\lambda_{s}}^{(r)}},
$$

where $\partial x_{\lambda_{s}}^{(r)} / \partial t_{\lambda j}{ }^{k}$ is defined as the corresponding coefficient of $t_{\lambda j}{ }^{k}$ in Eqs. (4.5)-(4.13). Let $\boldsymbol{\nabla}_{\mathbf{x}_{\lambda}}$ be the column gradient vector operator whose elements are the $\partial / \partial x_{\lambda_{s}}^{(r)}$ operators in the top-to-bottom order given by the left-to-right order in Eq. (C1). Let $\nabla_{\mathbf{t}_{\lambda}}$ be the analogous column gradient vector operator whose elements are the $\partial / \partial t_{\lambda j}{ }_{j}$ in the order of Eq. (C2). As a result, Eq. (C16) can be rewritten in the compact form

$$
\nabla_{\mathbf{t}_{\lambda}}=\tilde{\mathbf{M}} \nabla_{\mathbf{x}_{\lambda}}
$$

Therefore,

$$
\begin{aligned}
\nabla^{2} & =\tilde{\nabla}_{\mathbf{x}_{\lambda}} \nabla_{\mathbf{x}_{\lambda}} \\
& =\left(\tilde{\nabla}_{\mathbf{t}_{\lambda}} \mathbf{M}^{-1}\right)\left(\tilde{\mathbf{M}}^{-1} \nabla_{\mathbf{t}_{\lambda}}\right)=\tilde{\nabla}_{\mathbf{t}_{\lambda}}(\tilde{\mathbf{M}} \mathbf{M})^{-1} \nabla_{\mathbf{t}_{\lambda}} .
\end{aligned}
$$

The matrix

$$
\mathbf{B}=(\tilde{\mathbf{M}} \mathbf{M})^{-1}
$$

can be evaluated by the explicit expressions for $\mathbf{M}^{-1}$ given in Eqs. (C5)-(C14). However, it can be obtained instead from the unitarity of $\mathbf{M}$ and the property given by Eq. (C15). Indeed, let the rows and columns of an arbitrary $9 \times 9$ matrix be labeled, respectively, by the pairs $(k, j)$ and $\left(k^{\prime}, j^{\prime}\right)$, in the ordering of Eq. (C2), where the $k$ are the superscripts and the $j$ the subscripts. It is then possible to show, with the help of some straightforward matrix algebra, that the elements of $\mathbf{B}$ are given by

$$
B_{k j, k^{\prime} j^{\prime}}=(-1)^{k+j} \delta_{-k-j, k^{\prime} j^{\prime}}
$$

In other words, $\mathbf{B}$ is antidiagonal, and its antidiagonal elements are equal to $(-1)^{k+j}$. Replacement of the last two equations into Eq. (C18) gives the desired result,

$$
\nabla^{2}=\sum_{j, k=-1}^{1}(-1)^{k+j} \frac{\partial^{2}}{\partial t_{\lambda j}^{k} \partial t_{\lambda-j}^{-k}} .
$$

This same Laplacian would therefore also be obtained for any other unitary $\mathbf{M}$ [different from that specified by Eqs. (C5)-(C14)] satisfying Eq. (C15). It should be noted that although the right-hand side. of Eq. (C21) has nine terms, only five of them are distinct.

\section{APPENDIX D: HARMONIC POLYNOMIALS AND HYPERSPHERICAL HARMONICS}

The general theory of harmonic polynomials and hyperspherical harmonics is of central importance for this paper. We summarize here the properties that were used in deriving the basic recursion relations for the principal-axes-of-inertia hyperspherical harmonic $G$ functions, defined by Eq. (3.18) and given by Eqs. (4.27) and (4.34).

\section{Harmonic polynomials in m-dimensional space}

Consider the $\mathrm{R}^{m}$ space spanned by $\mathbf{x}=\left(x_{1}, x_{2}, \cdots, x_{m}\right)$ where the $x_{i}$ are real variables each spanning the full $-\infty$ to $+\infty$ domain. A general homogeneous polynomial of the nonnegative integer degree $n$ in $\mathbb{R}^{m}$ is defined as

$$
f_{(m)}^{n}(\mathbf{x})=\sum_{n_{i}} \mathcal{A}_{n_{1} n_{2} \cdots n_{m}} x_{1}^{n 1} x_{2}^{n 2} \cdots x_{m}^{n_{m}},
$$

where the $n_{i}(i=1-m)$ are non-negative integers. The sum extends over all such integers subject to the constraint,

$$
\sum_{i=1}^{m} n_{i}=n
$$

and the $\mathcal{A}$ are complex dimensionless constants. A harmonic polynomial $h_{(m)}^{n}(\mathbf{x})$ in $\mathbb{R}^{m}$ is a homogeneous polynomial of degree $n$ in that space which in addition satisfies the Laplace equation,

$$
\nabla_{(m)}^{2} h_{(m)}^{n}(\mathbf{x})=0,
$$

where $\nabla_{(m)}^{2}$ is the $m$-dimensional Laplacian defined by

$$
\nabla_{(m)}^{2}=\sum_{i=1}^{m} \frac{\partial^{2}}{\partial x_{i}^{2}} .
$$

Given an arbitrary homogeneous polynomial $f_{(m)}^{n}(\mathbf{x})$, the associated function $h_{(m)}^{n}(\mathbf{x})$ defined by 


$$
\begin{aligned}
h_{(m)}^{n}(\mathbf{x})= & \sum_{k=0}^{[n / 2]} \frac{(-1)^{k}(m+2 n-2 k-4) ! !}{(2 k) ! !(m+2 n-4) ! !} \\
& \times \rho_{m}^{(2 k)}\left(\nabla_{(m)}^{2}\right)^{k} f_{(m)}^{n}(\mathbf{x})
\end{aligned}
$$

is a harmonic polynomial, ${ }^{35}$ where $[n / 2]$ denotes the integer part of $n / 2$ and $\rho_{m}$ is the hyper-radius defined by

$$
\rho_{m}^{2}=\sum_{i=0}^{m} x_{i}^{2}
$$

\section{Dimensionless grand-canonical generalized angular momentum operators}

This operator is defined by ${ }^{36}$

$$
\hat{\wedge}_{(m)}^{2}=-\sum_{i=1}^{m} \sum_{j=1}^{i-1} \hat{\wedge}_{i j}^{2}
$$

where

$$
\hat{\wedge}_{i j}^{2}=x_{i} \frac{\partial}{\partial x_{j}}-x_{j} \frac{\partial}{\partial x_{i}} .
$$

From this definition one can easily derive the relation

$$
\nabla_{(m)}^{2}=\frac{1}{\rho_{m}^{m-1}} \frac{\partial}{\partial \rho_{m}} \rho_{m}^{m-1} \frac{\partial}{\partial \rho_{m}}-\frac{\hat{\wedge}_{(m)}^{2}}{\rho_{m}^{2}} .
$$

The dimensionless $\hat{\wedge}_{(m)}^{2}$ is related to the usual hyperangular momentum operator $\hat{\wedge}_{(m)}^{2}$ for a system of particles with $m$-spatial degrees of freedom by the proportionality constant $\hbar^{2}$.

Let $F_{(m)}^{n}$ be the function defined by

$$
F_{(m)}^{n}(\mathbf{y})=\frac{h_{(m)}^{n}(\mathbf{x})}{\rho_{m}^{n}},
$$

where

$$
\mathbf{y}=\left(y_{1}, y_{2}, \ldots, y_{m-1}\right)
$$

and

$$
y_{i}=x_{i} / \rho_{m} \quad(i=1,2, \ldots, m) .
$$

In view of Eq. (D6);

$$
\sum_{i=1}^{m} y_{i}^{2}=1 \text {, }
$$

and therefore only $m-1$ of the $m$ dimensionless variables $y_{i}$ are independent. In the right-hand side of Eq. (D11) we chose them arbitrarily to be the first $m-1$ of these quantities, but any set of $m-1$ of them could have been selected. It can easily be shown ${ }^{37}$ that the $F_{(m)}^{n}$ satisfy the partial differential equation,

$$
\hat{\wedge}_{(m)}^{2} F_{(m)}^{n}(\mathbf{y})=n(n+m-2) F_{(m)}^{n}(\mathbf{y}) .
$$

They are eigenfunctions of $\hat{\wedge}_{(m)}^{2}$ with eigenvalue $n(n+m$ $-2)$. These functions are called hyperspherical harmonics.

\section{Generalized hyperspherical coordinates}

Let $\boldsymbol{\alpha} \equiv\left(\alpha_{1}, \alpha_{2}, \ldots, \alpha_{m-1}\right)$ be a set of $m-1$ angles and $g_{i}(\boldsymbol{\alpha})(i=1,2, \ldots, m)$ a set of real functions of these angles subject to the constraint,

$$
\sum_{i=1}^{m} g_{i}^{2}(\boldsymbol{\alpha})=1 .
$$

In addition, let the hyper-radius $\rho_{m}$ and $\boldsymbol{\alpha}$ be related to $\mathbf{x}$ by

$$
x_{i}=\rho_{m} g_{i}(\boldsymbol{\alpha}) \quad i=1,2, \ldots, m .
$$

The angles $\boldsymbol{\alpha}$ are labeled hyperangles and the $m$ variables $\rho_{m}, \boldsymbol{\alpha}$ are called a set of generalized hyperspherical coordinates associated with $\mathbf{x}$. As a result of Eqs. (D12) and (D16), we have

$$
y_{i}=g_{i}(\boldsymbol{\alpha})(i=1,2, \ldots, m),
$$

which permits us to change from the independent variables $\mathbf{y}$ given by Eq. (D11) to the hyperangles $\boldsymbol{\alpha}$. Similarly, upon the $\mathbf{x}$ to the $\rho_{m}, \mathbf{y}$ variable transformation, the $\hat{\wedge}_{(m)}^{2}$ operator defined by Eqs. (D7) and (D8) is seen to be completely independent of $\rho_{m}$. As a result, Eq. (D14) can be rewritten [changing from $\hat{\wedge}_{(m)}^{2}$ to $\hat{\wedge}_{(m)}^{2}=\hbar^{2} \hat{\wedge}_{(m)}^{2}$ ] as

$$
\hat{\wedge}_{(m)}^{2} F_{(m)}^{n}(\boldsymbol{\alpha})=n(n+m-2) \hbar^{2} F_{(m)}^{n}(\boldsymbol{\alpha})
$$

and the hyperspherical harmonics $F_{(m)}^{n}(\boldsymbol{\alpha})$ are functions of the hyperangles only. The important property they satisfy is that they can be generated from hyperspherical polynomials by multiplication by $\left(\rho_{m}\right)^{-n}$. In the present paper involving tetraatomic systems we consider the particular case $m=9$ only.

\section{APPENDIX E: THE G HYPERSPHERICAL HARMONICS AS HOMOGENEOUS POLYNOMIALS}

Once the $F$ hyperspherical harmonics are explicitly defined by Eqs. (3.6)-(3.11), the $G$ hyperspherical harmonics are implicitly defined by Eqs. (3.16) and (3.17) or equivalently Eq. (3.18). We now wish to prove that these $G$ functions are homogeneous polynomials of degree $n$ in the variables $x, y$, and $z$ defined by Eqs. (4.15)-(4.17). This property will be very useful in obtaining the $G$ analytically.

Let us consider the function defined by Eq. (4.24). We already know that it is a harmonics polynomial of degree $n$ in the variables $t_{\lambda j}{ }^{k}$ and can therefore be written (omitting the primes in the indices) as

$$
\begin{aligned}
& h^{\Pi n{ }_{M_{J}} M_{L_{\lambda}}^{L} d} \underset{d}{D}\left(\rho, \chi, \boldsymbol{\Theta}_{\lambda}\right) \\
& \quad=\sum_{j_{1}, k_{1}, \cdots, j_{n}, k_{n}=-1}^{1} C_{j_{1} k_{1} \cdots j_{n} k_{n} k_{\lambda}} t_{\lambda_{1}}^{k_{1} \cdots t_{\lambda_{j_{n}}}} .
\end{aligned}
$$

Replacing this relation, as well as Eq. (4.14), in Eq. (4.24) permits us to write the corresponding $F^{\Pi n}$ as 


$$
\begin{aligned}
& F^{\Pi n{ }_{M_{J} M_{L_{\lambda}}}^{L}} \underset{d}{D}\left(\chi, \boldsymbol{\Theta}_{\lambda}\right) \\
& =\frac{1}{\rho^{n}} h^{\Pi n} \underset{M_{J} M_{L_{\lambda}}}{L} \stackrel{D}{d}\left(\chi, \boldsymbol{\Theta}_{\lambda}\right) \\
& =(-1)^{n \chi} \sum_{j_{1}, k_{1}, \cdots, j_{n}, k_{n}=-1}^{1} \sum_{p_{1} q_{1} \cdots p_{n} q_{n}=-1}^{1} C_{j_{1} k_{1} \cdots j_{n} k_{n}} \\
& \times \prod_{i=1}^{n} D_{k_{i} p_{i}}^{1}\left(\mathbf{a}_{\lambda}\right) \prod_{m=1}^{n} D_{q_{m} j_{m}}^{1}\left(\boldsymbol{\delta}_{\lambda}\right) \prod_{s=1}^{n} \bar{t}_{p_{s}}^{q_{s}}(\theta, \phi) .
\end{aligned}
$$

The products of the Wigner rotation functions $D_{k_{i} p_{i}}^{1}\left(\mathbf{a}_{\lambda}\right)$ can be expressed, by successive application of the ClebschGordan series, ${ }^{25}$ in terms of the $D_{M_{J} \Omega_{J_{\lambda}}}\left(\mathbf{a}_{\lambda}\right)$ functions and appropriate Clebsch-Gordan coefficients. A similar statement is valid for the $D_{q_{m} j_{m}}^{1}\left(\boldsymbol{\delta}_{\lambda}\right)$. As a result, we get

$$
\begin{aligned}
& F^{\Pi n} \underset{M_{J} M_{L_{\lambda}}^{L}}{\stackrel{D}{d}}\left(\chi, \Theta_{\lambda}\right) \\
& =(-1)^{n \chi} \sum_{\Omega_{J_{\lambda}}=-J}^{J} \sum_{\Omega_{L_{\lambda}}=-L}^{L} \\
& \times D_{M_{J} \Omega_{J_{\lambda}}}^{J}\left(\mathbf{a}_{\lambda}\right) D_{\Omega_{L_{\lambda}} M_{L_{\lambda}}}^{L}\left(\boldsymbol{\delta}_{\lambda}\right) G^{n}{ }_{\Omega_{J_{\lambda}} \Omega_{L_{\lambda}}}^{L} \underset{d}{D}(\theta, \phi),
\end{aligned}
$$

where

$$
\Omega_{J_{\lambda}}=\sum_{i=1}^{n} p_{i}, \quad \Omega_{L_{\lambda}}=\sum_{i=1}^{n} q_{i},
$$

and $G_{\Omega_{J_{\lambda}}}^{n} \Omega_{L_{\lambda}}^{L} \underset{d}{D}(\theta, \phi)$ is a linear combination of the products of $n \bar{t}_{p_{s}}^{q_{s}}(\theta, \phi)$ functions. In other words, this $G$ is a homogeneous polynomial of degree $n$ of the five variables $\bar{t}_{p}^{q}(\theta, \phi) \quad(p, q=-1,0,1 ; p+q=-2,0,2) \quad$ defined by Eqs. (4.15)-(4.17). This means, equivalently, that $G^{n}{ }_{\Omega_{J_{\lambda}}}^{J}{ }_{\Omega_{L_{\lambda}}}^{L}{ }_{d}^{D}(\theta, \phi)$ is a homogeneous polynomial of degree $n$ of the three variables $x, y$, and $z$, also defined by those equations, Q.E.D.

As described in the last paragraph of Sec. IV C, it is necessary to apply analytically the operator $\hat{K}^{2}+\hat{B}$, defined by Eqs. (2.18) and (2.19), to the $\left[\mathcal{G}^{n+1}{ }_{\Omega_{J_{\lambda}}}^{J} \Omega_{L_{\lambda}}^{L}(\theta, \phi)\right]_{J^{\prime} L^{\prime} d^{\prime}}$ functions defined by Eq. (4.27) and expressed as homogeneous polynomials in the three variables $x, y, z$. Using the relations between these three variables and $\theta, \phi$ given by Eqs. (4.15)-(4.17), it is straightforward to derive the expression,

$$
\begin{aligned}
\hat{K}^{2}+\hat{B}= & \frac{-\hbar^{2}}{\left(1-z^{2}\right) g(x, y, z)}\left\{\left[x z \frac{\partial}{\partial x}+y z \frac{\partial}{\partial y}\left(z^{2}-1\right) \frac{\partial}{\partial z}\right]\right. \\
& \times g(x, y, z)\left[x z \frac{\partial}{\partial x}+y z \frac{\partial}{\partial y}\left(z^{2}-1\right) \frac{\partial}{\partial z}\right] \\
& \left.+\left(y \frac{\partial}{\partial x}-x \frac{\partial}{\partial y}\right) g(x, y, z)\left(y \frac{\partial}{\partial x}-x \frac{\partial}{\partial y}\right)\right\},
\end{aligned}
$$

where $g(x, y, z)$ is the function defined by Eq. (5.15) but written in terms of the $x, y, z$ variables as

$$
g(x, y, z)=4 x y\left[(x+y)^{2}-z^{2}\right]\left[(x-y)^{2}-z^{2}\right] .
$$

Equations (E5) and (E6) permit all the algebraic manipulations performed by Mathematica and described in Sec. IV C to be done using these variables only.

\section{APPENDIX F: DERIVATION OF THE HYPERSPHERICAL HARMONICS $G^{n} \underset{ \pm n \pm n}{n} n_{d}^{D}$ AND $G^{n} \underset{ \pm n \mp n}{n} n d$}

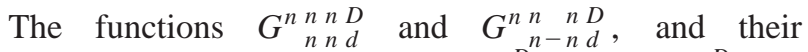
symmetry-related counterparts $G^{n} \begin{array}{r}n \\ -n-n d\end{array}$ and $G^{n} \begin{array}{r}n n D \\ -n n d\end{array}$, are particularly simple and are given by Eqs. (6.7) and (6.8). These expressions can be obtained by an analytical derivation without resorting to the Mathematica implementation of the recursion relations reported in this paper. This derivation is given below.

The functions $\left(t_{\lambda \pm 1}\right)^{n}$ have very useful properties that serve as the basis of this derivation. Use of Eq. (4.19) shows that their Laplacian is zero, and therefore that they are harmonic polynomials of degree $n$. As a result of Eqs. (D10) and (D18), we conclude that the related functions,

$$
\mathrm{F}_{ \pm}^{(n)}\left(\chi, \boldsymbol{\Theta}_{\lambda}\right)=\frac{\left[t_{\lambda \pm 1}{ }^{1}\left(\chi, \rho, \boldsymbol{\Theta}_{\lambda}\right)\right]^{n}}{\rho^{n}}
$$

are eigenfunctions of $\hat{\wedge}^{2}$ with an eigenvalue $n(n+7) \hbar^{2}$. Let us show that $\mathrm{F}_{ \pm}^{(n)}$ are also eigenfunctions of $\hat{J}^{2}, \hat{J}_{z}^{s f}, \hat{L}^{2}$, and $\hat{L}_{\lambda_{3}}^{b f}$ with eigenvalues $n(n+1) \hbar^{2}, n \hbar, n(n+1) \hbar^{2}$, and $\pm n \hbar$, respectively, and therefore that

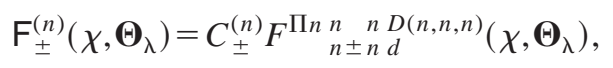

where $C_{ \pm}^{(n)}$ are proportionality constants. As a result of Eq. (4.14) for $j= \pm 1$ and $k=1$ we see that Eq. (F2) is indeed valid for $n=1$. We will now show by induction that this is true for an arbitrary $n$. We use the identity

$$
\mathrm{F}_{ \pm}^{(n)}=\mathrm{F}_{ \pm}^{(1)} \mathrm{F}_{ \pm}^{(n-1)}
$$

which is an immediate consequence of Eq. (F1). Assuming that Eq. (F2) is valid for $n-1$, we have

$$
\begin{aligned}
\mathrm{F}_{ \pm}^{(n-1)}= & C_{ \pm}^{(n-1)} \mathcal{N}^{n-1} n-1 n-1 \underset{d}{D(n-1, n-1, n-1)}(-1)^{(n-1) \chi} \\
& \times \sum_{\Omega_{J_{\lambda}}=-(n-1) \Omega_{L_{\lambda}}=-(n-1)}^{n-1} \sum_{n-1 \Omega_{J_{\lambda}}}^{n-1}\left(\mathbf{a}_{\lambda}\right) D_{\Omega_{L_{\lambda}}^{n-1} \pm(n-1)}^{n-1} \\
& \times\left(\boldsymbol{\delta}_{\lambda}\right) G^{n-1} \begin{array}{l}
n-1 \\
\Omega_{J_{\lambda}} \Omega_{L_{\lambda}} d
\end{array}
\end{aligned}
$$

In addition, since we already know that Eq. $(\mathrm{F} 2)$ is valid for $n=1$, we have

$$
\begin{aligned}
\mathrm{F}_{ \pm}^{(1)}= & C_{ \pm}^{(1)} \mathcal{N}^{111} \underset{d}{D(1,1,1)}(-1)^{\chi} \\
& \times \sum_{p=-1}^{1} \sum_{q=-1}^{1} D_{1 p}^{1}\left(\mathbf{a}_{\lambda}\right) D_{q \pm 1}^{1}\left(\boldsymbol{\delta}_{\lambda}\right) G_{p q d}^{1}{ }_{p}^{1} 1 D_{d}^{(1,1,1)}(\theta, \phi),
\end{aligned}
$$

where

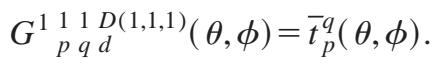


Replacement of Eqs. (F4)-(F6) into Eq. (F3) and use of the Clebsch-Gordan series for the products $D_{n-1 \Omega_{J_{\lambda}}}^{n-1}\left(\mathbf{a}_{\lambda}\right) D_{1 p}^{1}\left(\mathbf{a}_{\lambda}\right)$ and $D_{\Omega_{L_{\lambda}} \pm(n-1)}^{n-1}\left(\boldsymbol{\delta}_{\lambda}\right) D_{q \pm 1}^{1}\left(\boldsymbol{\delta}_{\lambda}\right)$ results in

$$
\begin{aligned}
& \mathrm{F}_{ \pm}^{(n)}=C_{ \pm}^{(1)} C_{ \pm}^{(n-1)} \mathcal{N}^{1} 111 \underset{d}{D(1,1,1)} \\
& \times \mathcal{N}^{n-1} n-1 n-1 \underset{d}{D(n-1, n-1, n-1)}(-1)^{n \chi} \\
& \times \sum_{\Omega_{J_{\lambda}}=-n}^{n} \sum_{\Omega_{L_{\lambda}}=-n}^{n} D_{n \Omega_{J_{\lambda}}}^{n}\left(\mathbf{a}_{\lambda}\right) D_{\Omega_{L_{\lambda} \pm(n)}}^{n}\left(\boldsymbol{\delta}_{\lambda}\right) \\
& \times P^{n}{ }_{\Omega_{J_{\lambda}} \Omega_{L_{\lambda}}}(\theta, \phi),
\end{aligned}
$$

where $P^{n} \Omega_{J_{\lambda}} \Omega_{L_{\lambda}}$ is a homogeneous polynomial of degree $n$ in the $\bar{t}_{r}^{s}(\theta, \phi)$ functions [defined by Eqs. (4.15)-(4.16)] and is given explicitly by

$$
\begin{aligned}
P_{\Omega_{J_{\lambda}} \Omega_{L_{\lambda}}=} & \sum_{p=-1}^{1} \sum_{q=-1}^{1} C\left(n-11 n ; \Omega_{J_{\lambda}}-p p \Omega_{J_{\lambda}}\right) \\
& \times C\left(n-11 n ; \Omega_{L_{\lambda}}-q q \Omega_{L_{\lambda}}\right) \bar{t}_{p}^{q}(\theta, \phi) \\
& \times G^{n-1} \begin{array}{c}
n-1 \\
\Omega_{J_{\lambda}}-p \Omega_{L_{\lambda}}^{n-1} \underset{d}{D(n-1, n-1, n-1)}(\theta, \phi)
\end{array}
\end{aligned}
$$

and is the same for both $F_{+}^{(n)}$ and $F_{-}^{(n)}$. Examination of Eq. (F7) shows that the $F_{ \pm}^{(n)}$ are indeed eigenfunctions of the operators listed after Eq. (F1), having the eigenvalues indicated, Q.E.D. As a result, Eq. (F2) is indeed valid, and furthermore we can also write, for all allowed $\Omega_{J_{\lambda}}$ and $\Omega_{L_{\lambda}}$,

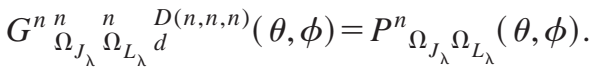

Setting $\Omega_{J_{\lambda}}=n$ and $\Omega_{L_{\lambda}}= \pm n$ in this expression produces the rather simple relation

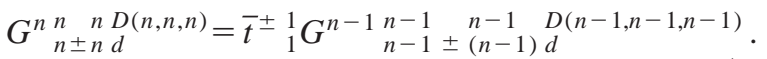

We know, from the remarks preceding Eq. (4.37), that $D(1,1,1)=1$. For $n=2$, Eq. (F11) furnishes $D(2,2,2)=1$, because, for this case, the product in its r.h.s. is unique, i.e., nondegenerate. By induction we conclude that, in general,

$$
D(n, n, n)=1 \text {, }
$$

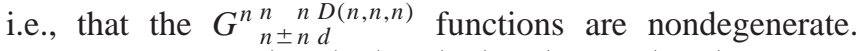
Substituting Eqs. (4.15), (4.16), (4.37), and (4.38) into Eq. (F10) for $n=2$, followed by induction over $n$, together with the symmetry relation Eq. (3.26), leads in a straightforward manner, to the desired simple expression,

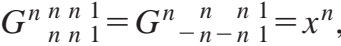

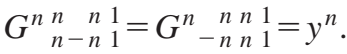

${ }^{1}$ J. M. Launay and M. le Dourneuf, Chem. Phys. Lett. 163, 178 (1989); 169, 473 (1990)

${ }^{2}$ J. M. Launay, Theor. Chim. Acta 79, 183 (1991); J. M. Launay and S. B. Pådkjær, Chem. Phys. Lett. 181, 95 (1991).

${ }^{3}$ S. Branchett, S. B. Pådkjær, and J. M. Launay, Chem. Phys. Lett. 208, 523 (1993).

${ }^{4}$ Y. S. M. Wu, A. Kuppermann, and B. Lepetit, Chem. Phys. Lett. 186, 319 (1991).

${ }^{5}$ Y. S. M. Wu and A. Kuppermann, Chem. Phys. Lett. 201, 178 (1993)

${ }^{6}$ A. Kuppermann and Y. S. M. Wu, Chem. Phys. Lett. 205, 577 (1993).

${ }^{7}$ U. Manthe, T. Seideman, and W. H. Miller, J. Chem. Phys. 99, 10078 (1993).

${ }^{8}$ D. Neuhauser, J. Chem. Phys. 100, 9272 (1994)

${ }^{9}$ D. H. Zhang and J. Z. H. Zhang, J. Chem. Phys. 101, 1146 (1994).

${ }^{10}$ D. H. Zhang and J. C. Light, J. Chem. Soc., Faraday Trans. 93, 691 (1997).

${ }^{11}$ D. H. Zhang and J. C. Light, J. Chem. Phys. 105, 1291 (1996).

${ }^{12}$ D. H. Zhang and J. C. Light, J. Chem. Phys. 104, 4544 (1996).

${ }^{13}$ D. H. Zhang and S. Y. Lee, J. Chem. Phys. 109, 2708 (1998).

${ }^{14}$ H. Szichman and M. Baer, J. Chem. Phys. 101, 2081 (1994).

${ }^{15}$ H. Szichman and M. Baer, Chem. Phys. Lett. 242, 285 (1995).

${ }^{16}$ S. K. Pogrebnya, J. Palma, D. C. Clary, and J. Echave, Phys. Chem. Chem. Phys. 2, 693 (2000).

${ }^{17}$ A. Kuppermann, J. Phys. Chem. A 101, 6368 (1997).

${ }^{18}$ V. Aquilanti and S. Cavalli, J. Chem. Soc., Faraday Trans. 93, 801 (1997).

${ }^{19}$ R. G. Littlejohn, K. A. Mitchell, and V. Aquilanti, Phys. Chem. Chem. Phys. 1, 1259 (1999).

${ }^{20}$ W. Zickendraht, J. Math. Phys. 10, 30 (1968).

${ }^{21}$ J. L. Ballot and M. Fabre de la Ripelle, Ann. Phys. (N.Y.) 127, 62 (1980).

${ }^{22}$ Y. F. Smirnov and K. V. Shitikova, Sov. J. Part. Nucl. 8, 344 (1977).

${ }^{23}$ C. D. Lin, Phys. Rev. A 25, 76 (1982); 26, 2305 (1982); 27, 22 (1983).

${ }^{24}$ J. S. Avery, Hyperspherical Hamonics (Kluwer, Dordrecht, 1989).

${ }^{25}$ A. S. Davydov, Quantum Mechanics (Addison-Wesley, Reading, 1965), pp. 154-156. In particular, the Clebsch-Gordan series is given by Eq. (43.19).

${ }^{26}$ A. Kuppermann, in Dynamics of Molecule and Chemical Reactions, edited by R. E. Wyatt and J. Z. H. Zhang (Dekker, New York, 1996), pp. 411472.

${ }^{27}$ M. E. Rose, Elementary Theory of Angular Momentum (Wiley, New York, 1957), p. 33

${ }^{28}$ S. Wolfram, Mathematica, 2nd ed. (Addison-Wesley, Reading, 1992).

${ }^{29}$ Reference 24, p. 35 with $d=9$ and $\lambda=n$.

${ }^{30} \mathrm{D}$. Wang and A. Kuppermann (to be published).

${ }^{31}$ G. C. Schatz, J. Chem. Phys. 74, 1133 (1981); D. H. Zhang and S. Y. Lee, ibid. 110, 4435 (1999).

${ }^{32}$ G. C. Schatz and H. Elgersma, in Potential Energy Surface and Dynamics Calculations, edited by D. G. Truhlar (Plenum, New York, 1981), pp. 311-328

${ }^{33}$ In Ref. 24 , p. 8.

${ }^{34}$ In Ref. 24, p. 11.

${ }^{35}$ In Ref. 24, p. 12.

${ }^{36}$ L. M. Delves, Nucl. Phys. 9, 39 (1959); 20, 275 (1960).

${ }^{37}$ A. Kuppermann, in Advances in Molecular Vibrations and Collision Dynamics, edited by J. M. Bowman (JAI, Greenwich, 1994), pp. 117-186. 